\title{
A DECIDABLE FRAGMENT OF THE ELEMENTARY THEORY OF THE LATTICE OF RECURSIVELY ENUMERABLE SETS
}

BY

\author{
M. LERMAN AND R. I. SOARE ${ }^{1}$
}

\begin{abstract}
ABSTRACr. A natural class of sentences about the lattice of recursively enumerable sets modulo finite sets is shown to be decidable. This class properly contains the class of sentences previously shown to be decidable by Lachlan. New structure results about the lattice of recursively enumerable sets are proved which play an important role in the decision procedure.
\end{abstract}

0. Introduction. Much of the recent work dealing with $\mathcal{E}$, the lattice of recursively enumerable sets, has dealt with global properties of $\mathcal{E}$ such as automorphisms and decidability, rather than local properties of $\mathcal{E}$, i.e., properties of definable classes of recursively enumerable sets. Two of the major results are Lachlan's [3] decision procedure for a natural fragment of the elementary theory of $\mathcal{E}^{*}$, the quotient lattice of $\mathcal{E}$ by the ideal of finite sets, Soare's result [15] on the existence of automorphisms carrying any maximal set into any other maximal set. More recently, Shore [13] has determined the definable automorphism bases for $\mathcal{E}$. These global results have inspired new local results, in that they have naturally led to the discovery of important new $\mathcal{E}$-definable classes of recursively enumerable sets whose properties have been investigated. Lachlan's result led to the discovery of small sets, Soare's result led to the discovery of $d$-simple sets, and Shore's result led to the discovery of nowhere simple sets; the first two of these classes have been studied by Lerman and Soare [8], and the third class by Shore [14]. The class of $d$-simple sets proved to be of particular importance, in that it led to the refutation of conjectures of Martin and Shoenfield which imply that the degrees of elements of any $\mathcal{E}$-definable class can be characterized by a finite set of equalities and inequalities involving the jumps of those degrees. Evidence for these conjectures included Martin's result [9] that $a$ is the degree of a maximal set if and only if $a^{\prime}=0^{\prime \prime}$, and results of Lachlan [2] and Shoenfield [12] that $a$ is the degree of an atomless set if and only if $a^{\prime \prime}>0$ ".

In this paper, we give a decision procedure for a larger fragment of the

Received by the editors April 14, 1978 and, in revised form, November 3, 1978.

AMS (MOS) subject classifications (1970). Primary 02F25; Secondary 02 G05.

Key words and phrases. Recursively enumerable sets, decision procedure, maximal sets, $r$-maximal sets, hyperhypersimple sets.

'The authors were partially supported by NSF grants MCS 76-07258 and MCS 77-01965, respectively. Some of the work on this paper was done while the first author was visiting the University of Illinois at Chicago Circle. 0002-9947/80/0000-0001/\$10.25 
elementary theory of $\mathcal{E}^{*}$ than did Lachlan. Such a decision procedure has previously been given by Lerman [6] for $\mathcal{E}^{*}(\alpha)$ for various admissible ordinals $\alpha$, where $\mathcal{E}^{*}(\alpha)$ is the analogue of $\mathcal{E}^{*}$ in $\alpha$-recursion theory. Our procedure led naturally to the discovery of the $\delta$-definable class of recursively enumerable sets which have $r$-maximal major subsets. This class has been studied by Lerman, Shore, and Soare [7], and it is important because it can be used to show that there are atomless hyperhypersimple sets $H_{1}$ and $H_{2}$ such that no automorphism of $\mathcal{E}$ carries $H_{1}$ to $H_{2}$.

The fragment of the elementary theory of $\mathcal{E}^{*}$ which we show to be decidable is the $\forall \exists$ theory of $\mathcal{E}^{*}$ in the language used by Lachlan [3] with an additional one-place relation symbol Max distinguishing the maximal sets. Our motivation for studying this class of sentences is that we view it as a preliminary step to finding a decision procedure for the $\exists \forall \exists$ sentences of Lachlan's language which hold in $\mathcal{E}^{*}$. Lachlan showed that $d$-lattices, i.e., ordered pairs $(L, A)$ where $L$ is a lattice and $A$ is the boolean algebra generated by $L$, play a natural role in the decision procedure and reduced the $\forall \exists$ decision problem to the following: Given finite $d$-lattices $(L, A)$, $\left(L_{1}, A_{1}\right), \ldots,\left(L_{k}, A_{k}\right)$, such that each $\left(L_{i}, A_{i}\right)$ is a refinement of $(L, A)$, when is it true that for all sub- $d$-lattices $(\mathcal{L}, \mathbb{Q})$ of $\left(\mathcal{E}^{*}, \mathbb{Q}^{*}\right)$ such that $(\mathfrak{L}, \mathbb{Q}) \simeq$ $(L, A)$, there exists a sub-d-lattice $\left(\mathcal{L}^{\prime}, \mathbb{Q}^{\prime}\right)$ of $\left(\mathcal{E}^{*}, \mathbb{Q}^{*}\right)$ such that one of the diagrams

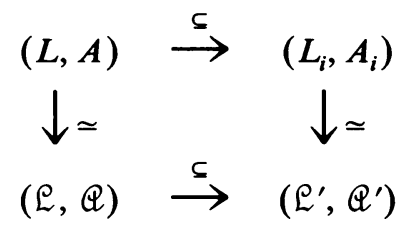

Figure 1

commutes? He produces finitely many canonical realizations $(\mathcal{L}, \mathcal{Q})$ of $(L, A)$ which provide necessary conditions for the diagram of Figure 1 to commute. He then proves a refinement theorem which shows that these necessary conditions are indeed sufficient.

In order to extend the decision procedure, one would now ask for definable necessary conditions given $(L, A),\left(L_{i}, A_{i}\right)$, and $(\mathcal{Q}, \mathbb{Q})$ as in Figure 1 , for the existence of $\left(\mathscr{L}^{\prime}, \mathbb{Q}^{\prime}\right) \subseteq\left(\mathcal{E}^{*}, \mathbb{Q}^{*}\right)$ such that the diagram of Figure 1 commutes. The necessary conditions provided by Lachlan point out a definable obstruction preventing us from obtaining such a refinement $\left(\mathcal{Q}^{\prime}, \mathbb{Q}^{\prime}\right)$. If we now add this definable obstruction to the language, and decide the $\forall \exists$ theory of $\left(\mathcal{E}^{*}, \mathbb{Q}^{*}\right)$ in the new language, we can repeat this procedure. Our hope is that there is a recursive language whose $\forall \exists$ theory is decidable which includes all such definable obstructions, and which admits an elimination of quantifiers transforming a $\exists \forall \exists$ sentence in the old language into a $\forall \exists$ sentence in the new language. As there are many possible obstructions, it may be impracticable to carry out this procedure. The simplest such obstruction, maximal sets, is treated in this paper. Our aim is to show that there is hope 
that the program is correct. In fact, our decision procedure also works if we add both Max and Hhs to Lachlan's language, where Hhs is a one-place relation symbol distinguishing the hyperhypersimple sets.

The outline of the paper is as follows: In $\$ 1$, we introduce the definitions and theorems about $d$-lattices which we will need, and, in $\$ 2$, we introduce the definitions and theorems about r.e. sets which we will need. We construct canonical realizations and obtain necessary conditions in $\$ 3$. In $\$ 4$, we prove the needed refinement theorems, and give the decision procedure. The reader will find an understanding of [3] very helpful.

1. $d$-lattices. All lattices considered in this paper will be distributive, with unequal least and greatest elements. $\subseteq$ will denote the lattice ordering, and $U$ and $\cap$ will denote the least upper bound and greatest lower bound operations of the lattice. 0 and 1 will denote the least and the greatest elements of the lattice unless we are dealing with sublattices of the lattice of r.e. sets, in which case we use $\varnothing$ for 0 and $\omega$ for 1 . Any distributive lattice with 0 and 1 generates a unique boolean algebra $A$. We will use the same symbols for constants, relations, and functions on $A$ as are used for $L$. In addition,' will denote the complementation operation on $A$. A d-lattice is a pair $(L, A)$ where $L$ is a distributive lattice and $A$ is the boolean algebra generated by $L$.

The $d$-lattice $(L, A)$ is said to be separated, if for all $a, b, c \in L$ such that $a \cup b=c$, there exist $a_{1}, b_{1} \in L$ such that $a_{1} \subseteq a, b_{1} \subseteq b, a_{1} \cup b_{1}=c$, and $a_{1} \cap b_{1}=0$. The $d$-lattice $\left(L_{1}, A_{1}\right)$ is said to be a sub-d-lattice of the $d$-lattice $(L, A)\left(\left(L_{1}, A_{1}\right) \subseteq(L, A)\right)$ if $A_{1}$ is a subalgebra of $A$ and $L_{1}=L \cap A_{1} \cdot(L, A)$ is said to be a refinement of $\left(L_{1}, A_{1}\right)$ if $\left(L_{1}, A_{1}\right) \subseteq(L, A)$. If $b \in L, b \neq 0$, then $(L, A) \mid b$ is the $d$-lattice $\left(L_{1}, A_{1}\right)$ where $L_{1}=\{c \in L: c \subseteq b\}, A_{1}=\{a \in$ $A: a \subseteq b\}$ and the ordering on $\left(L_{1}, A_{1}\right)$ is the same as that on $(L, A)$ for elements of $A_{1}$.

The $d$-lattices $(L, A)$ and $\left(L_{1}, A_{1}\right)$ are said to be isomorphic $((L, A) \simeq$ $\left.\left(L_{1}, A_{1}\right)\right)$ if there is an isomorphism $\psi: A \simeq A_{1}$ such that $\psi: L \rightarrow L_{1}$ is also an isomorphism. If $b \in L, b \neq 0$, and there is an isomorphism $\psi$ : $\left(L_{1}, A_{1}\right) \stackrel{\cong}{\rightarrow}\left(L_{2}, A_{2}\right) \subseteq(L, A) \mid b$, then we call $\psi$ an embedding of $\left(L_{1}, A_{1}\right)$ into $(L, A)$ and say that $\left(L_{1}, A_{1}\right)$ is embeddable into $(L, A)\left(\left(L_{1}, A_{1}\right) \hookrightarrow(L, A)\right)$. The embeddings $\psi:\left(L_{1}, A_{1}\right) \hookrightarrow(L, A)$ and $\xi:\left(L_{2}, A_{2}\right) \hookrightarrow(L, A)$ are said to be equivalent over $(L, A)$ if there is an isomorphism $\eta:\left(L_{1}, A_{1}\right) \simeq\left(L_{2}, A_{2}\right)$ such that the diagram

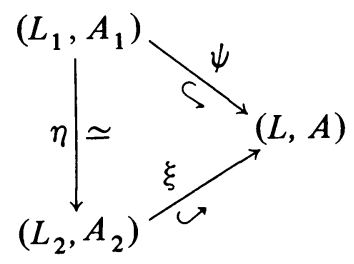

Figure 1.1 
commutes. The reader should note that our definition of $d$-lattice embedding is not the usual one. Rather, it is that of a map generated by a lattice embedding which preserves 0 but not necessarily 1 .

A component $\left(L_{1}, A_{1}\right)$ of $(L, A)$ is a $d$-lattice of the form $(L, A) \mid b$ where $b \neq 0, b \in L, b^{\prime} \in L$. Let $\left(L_{1}, A_{1}\right)$ and $\left(L_{2}, A_{2}\right)$ be two $d$-lattices. Their direct product $\left(L_{1}, A_{1}\right) \times\left(L_{2}, A_{2}\right)$ is the $d$-lattice $\left(L_{1} \times L_{2}, A_{1} \times A_{2}\right)$, where $L_{1} \times$ $L_{2}\left(A_{1} \times A_{2}\right)$ is the direct product of $L_{1}$ and $L_{2}\left(A_{1}\right.$ and $\left.A_{2}\right)$. A sequence of $d$-lattices $\left(\left(L_{1}, A_{1}\right), \ldots,\left(L_{k}, A_{k}\right)\right)$ is a decomposition of $(L, A)$ if $(L, A) \simeq$ $\left(L_{1}, A_{1}\right) \times \cdots \times\left(L_{k}, A_{k}\right) .(L, A)$ is said to be indecomposable if, for every decomposition of $(L, A)$ as above, $k=1$. Otherwise, $(L, A)$ is said to be decomposable.

The following lemma is stated in Lachlan [3].

LEMMA 1.1 (REPRESENTATION LEMMA). Every finite d-lattice has a decomposition into a finite number of indecomposable components. This decomposition is unique up to the order and isomorphism type of the components.

Let $b_{1}, \ldots, b_{k} \in L$ such that for all $i, j<k, b_{1} \neq 0$ and $b_{i} \cap b_{j}=0$. Let $b=\bigcup\left\{b_{i}: i \leqslant k\right\}$, and let $\left(L_{i}, A_{i}\right)=(L, A) \mid b_{i}$ for $i<k$. Let $(M, B)$ be isomorphic to a sub-d-lattice of $(L, A) \mid b$. For $i<k$, let $\psi_{i}:\left(L_{i}, A_{i}\right) \hookrightarrow(M, B)$. Define $L^{*}$ by $b \in L^{*}$ if and only if there is a $d \in M$ such that $b=\cup\{c$ : $\left.(\exists i<k)\left(c \in L_{i} \& \psi_{i}(c) \subseteq d\right)\right\}$ and $A^{*}$ by $a \in A^{*}$ if and only if there is a $d \in B$ such that $a=\bigcup\left\{c:(\exists i<k)\left(c \in A_{i} \& \psi_{i}(c) \subseteq d\right)\right\}$. Then if $\left(L^{*}, A^{*}\right)$ is a sub- $d$-lattice of $(L, A) \mid b$ which is isomorphic to $(M, B)$, it is called the fusion of the $\left(L_{i}, A_{i}\right)$ over $(M, B)$.

Fusion captures the following situation. Given a finite $d$-lattice $(M, B)$ and $k d$-lattices $\left(L_{1}, A_{1}\right), \ldots,\left(L_{k}, A_{k}\right)$ each of which is embeddable into $(M, B)$, classify all possible isomorphisms of $(M, B)$ onto a sub- $d$-lattice of $\mathrm{X}_{i=1}^{k}\left(L_{i}, A_{i}\right) .\left(L^{*}, A^{*}\right)$ is a fusion of the $\left(L_{i}, A_{i}\right)$ over $(M, B)$ exactly when it is an isomorphic copy of $(M, B)$ which is a sub-d-lattice of $\times_{i=1}^{k}\left(L_{i}, A_{i}\right) . \psi_{i}$ is then the natural restriction of the isomorphism $\psi:\left(L^{*}, A^{*}\right) \rightarrow(M, B)$ to $\left(L_{i}, A_{i}\right)$.

Lemma 1.2 (Fusion Lemma). For $i<k$, let $\left(L_{i}, A_{i}\right)=(L, A) \mid b_{i}$. Let $\left(L^{*}, A^{*}\right)$ be the sub-d-lattice of $(L, A)$ which is the fusion of the $\left(L_{i}, A_{i}\right)$ over $(M, B)$. Assume that $\left(L^{*}, A^{*}\right)$ is finite, $(N, C) \simeq\left(L^{*}, A^{*}\right)$, and $\left(N^{+}, C^{+}\right)$is a finite refinement of $(N, C)$. Then there exists a d-lattice $\left(L^{+}, A^{+}\right)$such that the diagram

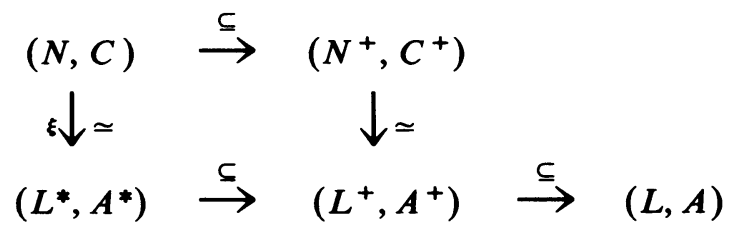

Figure 1.2

commutes if and only if for each $i<k$ there is a refinement $\left(L_{i}^{+}, A_{i}^{+}\right)$of 
$\left(L_{i}, A_{i}\right)$ with $\left(L_{i}^{+}, A_{i}^{+}\right) \subseteq(L, A) \mid b_{i}$ and embeddings $\psi_{i}:\left(L_{i}^{+}, A_{i}^{+}\right) \hookrightarrow\left(N^{+}, C^{+}\right)$ defined by $\psi_{i}(a)=d$ if $\xi(e)=b$ where $e$ is the least element of $C$ such that $d \subseteq e$ and $b$ is the least element of $\left(L^{*}, A^{*}\right)$ such that $a \subseteq b$, such that the fusion of the $\left(\mathrm{L}_{i}^{+}, \mathrm{A}_{i}^{+}\right)$over $\left(\mathrm{N}^{+}, \mathrm{C}^{+}\right)$is isomorphic to $\left(\mathrm{N}^{+}, \mathrm{C}^{+}\right)$.

Proof. Suppose that Figure 1.2 is a commutative diagram. Let $L_{i}^{+}=$ $L^{+}\left|b_{i}, A_{i}^{+}=A^{+}\right| b_{i}$. Then $\left(L_{i}^{+}, A_{i}^{+}\right)$has the desired properties. Conversely, if we let $\left(L^{+}, A^{+}\right)$be the fusion of the $\left(L_{i}^{+}, A_{i}^{+}\right)$over $\left(N^{+}, C^{+}\right)$, then the diagram of Figure 1.2 commutes.

Let $(L, A)$ be a finite $d$-lattice. An atom of $(L, A)$ is an element $a \in A$ such that $a \neq 0$ and for all $b \in A, 0<b \leqslant a$ implies $b=a$. A canonical generator of $(L, A)$ is a join irreducible element of $L$, i.e., an element $c \in L$ such that for all $a, b \in L$, if $a \cup b=c$ then $a=c$ or $b=c$. Note that the isomorphism type of $(L, A)$ is uniquely determined by the ordering of the canonical generators of $(L, A)$. Given any element $c \in A, L(c)$ will denote the least element $d \in L$ such that $c \subseteq d$. Note that if $c$ is an atom, then $L(c)$ is a canonical generator. Given a canonical generator $d, A(d)$ will denote the unique atom $a$ such that $a \subseteq d$ and $a \nsubseteq c$ for any canonical generator $c \subset d$. Given $c, d \in A$, we say that $c$ is within $d(c \preccurlyeq d)$ if $c \subseteq L(d) . \leqslant$ restricted either to atoms or to canonical generators yields a partial ordering. We write $c \prec d$ if $c \subseteq L(d)$ but $d \nsubseteq L(c)$. We say that $c$ is just within $d\left(c \prec_{j} d\right)$ if $c \prec d$ but for no $e \in A$ do we have $c \prec e \prec d$. An atom $a$ is said to be outermost (innermost) if there is no atom $b$ such that $a<b(b \prec a)$.

A path in the finite $d$-lattice $(L, A)$ is a finite sequence of atoms $a_{1}, \ldots, a_{k}$ such that $a_{1}$ is outermost and for all $i<k, a_{i+1} \prec_{j} a_{i}$. The rank of the finite $d$-lattice $(L, A)$ is $\left(n_{1}, \ldots, n_{h}\right)$ where $n_{i}$ is the number of paths of length $i$ and $h$ is the greatest $i$ such that $n_{i} \neq 0$. Ranks are well ordered by the definition $\left(n_{1}, \ldots, n_{h}\right)<\left(m_{1}, \ldots, m_{k}\right)$ if $k>h$ or if $k=h$ and

$$
(\exists x)\left(1<x<k \&(\forall y)\left(x<y<k \rightarrow n_{y}=m_{y}\right) \& n_{x}<m_{x}\right) \text {. }
$$

Let $(L, A) \simeq\left(L_{1}, A_{1}\right) \times \cdots \times\left(L_{k}, A_{k}\right)$ be a decomposition of $(L, A)$ into indecomposable components, arranged in order of decreasing ranks, where $\left(L_{i}, A_{i}\right)$ has rank $r_{i}$. The characteristic of $(L, A)$ is $\left(r_{1}, \ldots, r_{k}\right)$. Characteristics are well ordered by $\left(r_{1}, \ldots, r_{k}\right)<\left(s_{1}, \ldots, s_{m}\right)$ if either $(\exists x)(1<x<k \& 1$ $\left.<x<m \&(\forall y)\left(1<y<x \rightarrow r_{y}=s_{y}\right) \& r_{x}<s_{x}\right)$ or $k<m$ and $r_{x}=s_{x}$ for $1<x<k$. The following lemma is proved in Lachlan [3].

LEMMA 1.3. Any finite d-lattice $(L, A)$ has a finite separated refinement $\left(L^{*}, A^{*}\right)$ with characteristic $\leqslant$ the characteristic of $(L, A)$.

$\mathcal{E}$ will denote the lattice of r.e. sets, and $\mathcal{Q}$ will denote the boolean algebra generated by $\mathcal{E} .(\mathcal{E}, \mathbb{Q})$ is then a $d$-lattice. We let $\mathcal{E}^{*}\left(Q^{*}\right)$ be the quotient of $\mathcal{E}(\mathbb{Q})$ by the ideal of finite sets. Lachlan [4] shows that the elementary theories of $(\mathcal{E}, \mathbb{Q})$ and $\left(\mathcal{E}^{*}, \mathbb{Q}^{*}\right)$ are equidecidable, so nothing is lost in trying to obtain a decision procedure for the elementary theory of $\left(\mathcal{E}^{*}, \mathbb{Q}^{*}\right)$ instead of the elementary theory of $(\mathcal{E}, \mathbb{Q})$. 
Let $(L, A)$ be a finite $d$-lattice. A realization of $(L, A)$ is a sub- $d$-lattice $(\mathscr{P}, \mathscr{B})$ of $(\mathscr{G}, \mathscr{Q})$ with no finite atoms such that $(\mathscr{P}, \mathscr{B}) \simeq(L, A)$. If $(\mathscr{P}, \mathscr{B})$ $\subseteq(\mathcal{E}, \mathcal{Q})$ and $C$ is an r.e. set, then $(\mathscr{P}, \mathscr{B}) \cap C=(\mathscr{P} \cap C, \mathscr{B} \cap C)$ is the $d$-lattice for which

$$
\mathscr{P} \cap C=\{B:(\exists D \in \mathscr{P})(B=D \cap C)\}
$$

and

$$
\mathscr{B} \cap C=\{B:(\exists D \in \mathscr{B})(B=D \cap C)\} \text {. }
$$

The $d$-lattice $(\mathscr{P}, \mathscr{B}) \cap C$ with one component, where $(\mathscr{P}, \mathscr{B}) \subseteq(\mathcal{E}, \mathcal{Q})$, is said to be irreducible if given any r.e. set $D \subseteq C$ such that $C-D$ is r.e. and the outermost atom of $(\mathscr{P}, \mathscr{B}) \cap C$ is contained in $D$, then $(\mathscr{P}, \mathscr{B}) \cap D \simeq$ $(\mathscr{P}, \mathscr{B}) \cap C$. The finite $d$-lattice $(\mathscr{P}, \mathscr{B}) \cap C$ is said to be irreducible if each component of $(\mathcal{P}, \mathscr{B}) \cap C$ in any indecomposable representation of $(\mathscr{P}, \mathscr{B})$ $\cap C$ is irreducible. $(\mathscr{P}, \mathscr{B}) \cap C$ is reducible if it is not irreducible. (Warning: Reducible and decomposable are different.) The following lemma is proved by Lachlan [3].

LEMMA 1.4. Let $\left(\mathscr{P}^{*}, \mathscr{B}^{*}\right) \subseteq\left(\mathcal{E}^{*}, \mathbb{Q}^{*}\right)$, and let $c$ be the characteristic of $\left(\mathscr{P}^{*}, \mathscr{B}^{*}\right)$, with $\left(\mathscr{P}^{*}, \mathscr{B}^{*}\right)$ finite. Then there exists a finite separated refinement $\left(\mathscr{P}^{+}, \mathscr{B}^{+}\right) \subseteq\left(\mathcal{E}^{*}, \mathscr{Q}^{*}\right)$ of $(\mathscr{P}, \mathscr{B})$ with characteristic $<c$. Furthermore, given $\left(\mathscr{P}^{*}, \mathscr{B}^{*}\right)$, we can effectively enumerate a finite number of isomorphism types and for each such type, an isomorphism of $\left(\mathscr{P}^{*}, \mathscr{B}^{*}\right)$ into it, so that $\left(\mathscr{P}^{+}, \mathscr{B}^{+}\right)$ can be found in one of these types, with the isomorphism picking out $\left(\mathscr{P}^{*}, \mathscr{B}^{*}\right)$.

Our language for $d$-lattices $(L, A)$ will be the pure predicate calculus with equality together with the following symbols whose interpretations in $\left(\mathcal{E}^{*}, \mathbb{Q}^{*}\right)$ are given in parentheses: Two-place function symbols $\cup$ (union) and $\cap$ (intersection), a one-place function symbol' (complementation), a two-place relation symbol $\subseteq$ (ordering), a one-place relation symbol $L$ $(x \in L)$, a constant symbol 0 (least element), and a one-place predicate Max ( $x$ is a maximal set). Furthermore, quantifiers range only over r.e. sets. A diagram is a quantifier free formula $\sigma\left(x_{1}, \ldots, x_{n}\right)$ which can be interpreted as the complete diagram of some finite $d$-lattice. We will treat diagrams as if they were $d$-lattices.

We adopt the following notation. $\lambda x f(x, y)$ will denote the function of one variable $x$ with $y$ fixed. $\mu$ will denote the least number operator. If $f$ is a function, $\operatorname{dom}(f)$ will denote the domain of $f$ and $\operatorname{rng}(f)$ will denote the range of $f$. If $S \subseteq \operatorname{dom}(f)$, then $f \mid S$ will denote the restriction of $f$ to domain $S$. $f \mid x$ will denote the restriction of $f$ to domain $\{y: y<x\}$ for $x \in \omega$. For a set $S \subseteq \omega$ and $x \in \omega, S \mid x$ will denote $S \cap\{y: y<x\}, \operatorname{card}(S)$ will denote the cardinality of $S$, and $\max (S)$ will denote the greatest element of $S$ if $S$ is finite. $\left\{S_{i}: i \in I\right\}$ is said to be a partition of $S$ if $\cup\left\{S_{i}: i \in I\right\}=S$ and $\left\{S_{i}\right\}$ is a collection of pairwise disjoint sets. We say that

$$
\lim _{s} f(s)=n \quad \text { if }(\exists t)(\forall r)(r \geqslant t \rightarrow f(r)=n)
$$


and

$$
\lim _{s} f(s)=\infty \quad \text { if }(\forall m)(\forall t)(\exists r)(r \geqslant t \& f(r)>m)
$$

2. Recursively enumerable sets. This section contains the structure theory for $(\mathcal{E}, \mathbb{Q})$ needed for our decision procedure.

For $A, B \subseteq \omega$, we say that $A \subseteq \subseteq^{*} B$ if for all but finitely many $x \in \omega$, if $x \in A$ then $x \in B$. We say that $A={ }^{*} B$ if $A \subseteq \subseteq^{*} B$ and $B \subseteq \subseteq^{*} A$. Let $\left\{W_{e}\right.$ : $e<\omega\}$ be a standard enumeration of all the r.e. sets, with recursive approximation $\left\{W_{e}^{s}: e<\omega \& s<\omega\right\}$. We assume without loss of generality that for each $s$, there is a unique $e$ such that $W_{e}^{s+1}-W_{e}^{s} \neq \varnothing$. Let $W_{e} \backslash W_{n}$ denote $\left\{x:(\exists s)\left(x \in W_{e}^{s} \& x \notin W_{e}^{n}\right)\right\}$, and let $W_{e} \searrow W_{n}=\left(W_{e} \backslash W_{n}\right) \cap W_{n}$.

The following facts about $(\mathcal{E}, \mathcal{Q})$ are well known.

TheOrem 2.1. $(\mathcal{E}, \mathbb{Q})$ is separated.

THEOREM 2.2. For every infinite r.e. set $W,(\mathcal{E}, \mathbb{Q}) \mid W \simeq(\mathcal{E}, \mathbb{Q})$.

THEOREM 2.3. There exists an r.e. set which is not recursive.

Given an r.e. nonrecursive set $A$, we call $A_{1}, \ldots, A_{k}$ a splitting of $A$ if $\cup\left\{A_{i}: 1<i<k\right\}=A, A_{i} \cap A_{j}=\varnothing$ if $i \neq j$, and each $A_{i}$ is r.e. but not recursive. $C \subseteq \omega$ is said to be cohesive if $C \neq \neq^{*} \varnothing$ and for every r.e. set $W$, either $C \subseteq \subseteq^{*} W$ or $C \cap W={ }^{*} \varnothing . M \subseteq \omega$ is said to be maximal if $M$ is r.e. and $M^{\prime}$ is cohesive. The following well-known theorems were proved by Friedberg [1].

Theorem 2.4 (SPlitting TheORem). For any r.e. nonrecursive set $A$ and any $k<\omega$, there is a splitting $A_{1}, \ldots, A_{k}$ of $A$ such that

(2.1) for any r.e. set $W$ and $i \leqslant k$, if $W-A$ is not r.e. then $W-A_{i}$ is not r.e.

We call a splitting of $A$ satisfying (2.1) a Friedberg splitting.

Theorem 2.5 (MAXimal Set Theorem). There exists a maximal set.

Let $A$ and $B$ be r.e. sets. $A$ is said to be a major subset of $B$ if $A \subseteq B$, $B-A \neq^{*} \varnothing$, and for all r.e. sets $W$, if $B^{\prime} \subseteq W$ then $A^{\prime} \subseteq \subseteq^{*} W . A$ is said to be a small subset of $B$ if $A \subseteq B, B-A \neq^{*} \varnothing$, and for all r.e. sets $W, X$, if $X \cap(B-A) \subseteq W$ then $W \cup(X-B)$ is r.e. The following theorem was proved by Lachlan [3].

Theorem 2.6 (SMAll Major Subset Theorem). Let $B$ be an r.e. nonrecursive set. Then $B$ has a small major subset.

Let $D=A-C$ with $A$ and $C$ r.e. sets. $D$ is said to be pure d-r.e. if $D \neq^{*} \varnothing$ and for all $B \subseteq \omega$, if $B \neq^{*} \varnothing$ and $B \subseteq D$ then $B^{\prime}$ is not r.e. The following remark is easily verified.

Remark 2.7. Let $A \subseteq B$ be r.e. sets. Then $A$ is a major subset of $B$ if and only if $B-A$ is pure $d$-r.e. 
Let $K, H \subseteq \omega . H$ is said to be hyperhypersimple in $K$ (hhs in $K$ ) if $K-H \neq{ }^{*} \varnothing, H$ is r.e., and the lattice of r.e. sets $J$ such that $H \subseteq J \subseteq K$ under inclusion is a boolean algebra. $H$ is hyperhypersimple (hhs) if $H$ is hhs in $\omega . S \subseteq \omega$ is hyperhyperimmune (hhi) if $S^{\prime}$ is hhs. The following theorem was proved by Lachlan [4].

THEOREM 2.8. If $H$ is hhs and $K$ is hhs in $H$, then $K$ is hhs.

An r.e. set $A \neq * \varnothing$ is said to be atomless if $A$ does not have a maximal superset. A function $h: \omega \rightarrow \omega$ is $\Delta_{3}^{0}$ if there is a recursive function $h^{*}: \omega^{3} \rightarrow \omega$ such that for all $x, h(x)=\lim _{s} \lim _{t} h^{*}(s, t, x)$.

We will need a recursive enumeration $\left\{R_{e}^{i}: e<\omega \& i \in\{0,1\}\right\}$ of all pairs of pairwise disjoint recursive sets $R, S$ such that either $R \cup S$ is a finite initial segment of $\omega$, or $R=S^{\prime}$. For each such pair, there will be an $e$ such that $R=R_{e}^{0}$ and $S=R_{e}^{1}$. Such an enumeration can be obtained from the eumeration of pairs of r.e. sets. Let $\left(W_{e_{0}}, W_{e_{1}}\right)$ be the eth pair of r.e. sets. Define $x \in R_{e, s}^{i}$ if $W_{e_{0}}^{s} \cap W_{e_{1}}^{s}=\varnothing$, and for all $y<x, y \in W_{e_{0}}^{s} \cup W_{e_{1}}^{s}$, and $x \in W_{e}^{s} . R_{e}^{i}$ is then $\cup\left\{R_{e, s}^{i}: s<\omega\right\}$. The function $h: \omega \rightarrow\{0,1\}$ is said to be a preference function for the r.e. set $A$ if for all $e<\omega$,

$$
\bigcap\left\{R_{i}^{h(i)}: i<e \& R_{i}^{0} \cup R_{i}^{1} \text { infinite }\right\} \cap A^{\prime} \text { is infinite. }
$$

The construction of Lachlan [4] yields the following theorem as noted in Lerman, Shore, and Soare [7].

THEOREM 2.9. There exists an atomless hhs set $H$ with a $\Delta_{3}^{0}$ preference function.

The set $S \subseteq \omega$ is said to be $r$-cohesive if $S \neq{ }^{*} \varnothing$ and for all recursive sets $R$, either $S \subseteq \subseteq^{*} R$ or $S \cap R={ }^{*} \varnothing$. If $A$ and $B$ are r.e. sets with $A \subseteq B$ and $B-A \neq^{*} \varnothing$, then $A$ is said to be an $r$-maximal subset of $B$ if $B-A$ is $r$-cohesive. $A$ is $r$-maximal if $A$ is an $r$-maximal subset of $\omega$.

An r.e. set $Q$ is said to be quasimaximal of degree $n$ if there are maximal sets $M_{1}, \ldots, M_{n}$ such that $Q=\cap\left\{M_{i}: i<n\right\}$ and for all $i, j<n$, if $i \neq j$ then $M_{i} \neq M_{j} . S \subseteq \omega$ is said to be quasicohesive of degree $n$ if $S^{\prime}$ is quasimaximal of degree $n . Q \subseteq \omega$ is said to be quasimaximal (quasicohesive) if for some $n<\omega, Q$ is quasimaximal (quasicohesive) of degree $n$.

Define the relation $\equiv_{r}$ on $r$-maximal sets $B$ and $C$ by $B \equiv{ }_{r} C$ if and only if $B \cap C$ is $r$-maximal. Note that $B \equiv{ }_{r} C$ if and only if $B \cup C \neq \neq^{*} \omega$. It is easily verified that $\equiv_{r}$ is an equivalence relation. An r.e. set $Q$ is said to be quasi-r-maximal of degree $n$ if there are $r$-maximal sets $B_{1}, \ldots, B_{n}$ such that $Q=\cap\left\{B_{i}: i \leqslant n\right\}$ and for all $i, j \leqslant n$, if $i \neq j$ then $B_{i} Z_{r} B_{j} . S \subseteq \omega$ is said to be quasi-r-cohesive of degree $n$ if $S^{\prime}$ is quasi-r-maximal of degree $n$. $Q \subseteq \omega$ is said to be quasi-r-maximal (quasi-r-cohesive) if for some $n<\omega, Q$ is quasi-r-maximal (quasi-r-cohesive) of degree $n$.

$A \subseteq \omega$ is said to be immune if $A \neq \neq^{*} \varnothing$ and for any infinite r.e. set $W$, $W \cap A^{\prime} \neq{ }^{*} \varnothing . S \subseteq \omega$ is said to be simple if $S$ is r.e. and $S^{\prime}$ is immune. The following remark is well known and easily verified. 
REMARK 2.10. If $\boldsymbol{A}$ has any of the following properties, then $\boldsymbol{A}$ is immune: (a) $A$ is hhi; (b) $A$ is cohesive; (c) $A$ is $r$-cohesive; (d) $A$ is quasicohesive; (e) $A$ is quasi-r-cohesive; (f) $A$ is pure $d$-r.e.; (g) $A^{\prime}$ is atomless.

Let $A$ and $B$ be r.e. sets such that $A \subseteq B$ and $A \cup B^{\prime}$ is not r.e. Let $E_{1}, \ldots, E_{k}$ partition $B$. Then we call $E_{1}, \ldots, E_{k}$ an Owings splitting of $B$ above $A$ if for all $i<k$, the following holds:

(2.2) for all r.e. sets $W$, if $A \cup\left(W-E_{i}\right)$ is r.e., then $A \cup(W-B)$ is r.e. Note that (2.2) implies

(2.3) $A \cup E_{i}^{\prime}$ is not r.e.

and (2.3) implies

(2.4) $E_{i}-A$ is infinite.

Owings [10] constructs a splitting of $B-A$ satisfying (2.3) and hence (2.4). His construction, however, also yields (2.2). We reprove Owings' theorem here in the form which we will later need.

TheOREM 2.11. Let $A$ and $B$ be r.e. nonrecursive sets such that $A \subseteq B$ and $A \cup B^{\prime}$ is not r.e. Let $k \geqslant 1$. Then there exists an Owings splitting $E_{1}, \ldots, E_{k}$ of $B$ above $A$.

Proof. If $k=1$, the theorem is immediate setting $E_{1}=B$. Suppose that $k>1$. Fix one-one recursive enumerations $\left\{a_{s}: s<\omega\right\}$ of $A$ and $\left\{b_{s}: s<\omega\right\}$ of $B$. For each $i \leqslant k$ and $e, m<\omega$, establish the requirement specified by $i, e$, and $m$,

$$
A \cup\left(W_{e}-E_{i}\right)=W_{m} \rightarrow A \cup\left(W_{e}-B\right) \text { is r.e. }
$$

Well order these requirements into a recursive sequence $\left\{R_{j}: j<\omega\right\} . R_{j}$ is said to be satisfied if the condition it imposes holds.

We will define a recursive function $h: \omega^{2} \rightarrow \omega$ such that for each $s, \lambda j h(s, j)$ has infinite range. We say that $j$ potentially captures $b_{s}$ if $h(s, j)>b_{s}$ and $b_{s} \in W_{e}^{s} \cap W_{m}^{s}$. The least integer which potentially captures $b_{s}$ is said to capture $b_{s}$. If $j$ captures $b_{s}$ and $R_{j}$ is the requirement specified by $i, e$, and $m$, then we say that $b_{s}$ is targeted for $E_{i}$.

Let $A^{s}=\left\{a_{i}: i<s\right\}, B^{s}=\left\{b_{i}: i<s\right\}$, and $E_{i}^{s}=$ the set of numbers placed into $E_{i}$ before stage $s$ of the construction. We say that $R_{j}$, the requirement specified by $i, e$, and $m$, requires attention at stage $s$ if

$$
A^{s}\left|h(s, j) \cup\left(W_{e}^{s}\left|h(s, j)-E_{i}^{s}\right| h(s, j)\right)=W_{m}^{s}\right| h(s, j) .
$$

The construction. Set $h(0, j)=j$ for all $j<\omega$.

Stage $s$. If $b_{s}$ is not captured by any $j$, place $b_{s} \in E_{i}$. Otherwise, let $j$ capture $b_{s}$. Place $b_{s} \in E_{i}$ where $b_{s}$ is targeted for $E_{i}$. For all $j<\omega$, let

$$
h(s+1, j)= \begin{cases}h(s, j)+1 & \text { if } j<s \text { and } j \text { requires attention } \\ h(s, j) & \text { at stage } s, \\ \text { otherwise. }\end{cases}
$$

This completes the construction. For all $j<\omega$, let $h^{*}(j)=\lim _{s} h(s, j)$. Let $J=\left\{j: h^{*}(j)<\infty\right\} .\left\{E_{i}: i<k\right\}$ clearly partitions $B$. 
LEMMA 2.12. Let $j$ capture $b_{s}, j \notin J$. Then $b_{s} \in A$.

Proof. Let $R_{j}$ be specified by $i, e$, and $m$ and suppose that $j$ captures $b_{s}$. Then $b_{s} \in E_{i}^{s+1}-E_{i}^{s}$. Since $j \notin J, j$ requires attention at infinitely many stages, hence $A \cup\left(W_{e}-E_{i}\right)=W_{m}$. Since $j$ captures $b_{s}, b_{s} \in\left(W_{e}^{s}-E_{i}^{s}\right) \cap$ $W_{m}^{s} \subseteq W_{m}$. But $b_{s} \notin W_{e}-E_{i}$ and $b_{s} \in W_{m}$, so $b_{s} \in A$.

LEMMA 2.13. If $j \in J$ then $R_{j}$ is satisfied.

Proof. Let $R_{j}$ be specified by $i, e$, and $m$. Since $j \in J$, for all sufficiently large $t, j$ does not require attention at stage $t$. Hence for some $x<h^{*}(j)$, $x \in A \cup\left(W_{e}-A_{i}\right)$ if and only if $x \notin W_{m}$, so $R_{j}$ is satisfied.

LEMMA 2.14. If $j \notin J$, then $R_{j}$ is satisfied.

Proof. Fix $j \notin J$, and let $R_{j}$ be specified by $i$, e, and $m$. Since $h^{*}(j)=\infty$, we must have $A \cup\left(W_{e}-W_{i}\right)=W_{m}$. Let

$$
Z=\left\{x:(\exists t)\left(x<h(t, j) \& x \in W_{e}^{t} \cap W_{m}^{t} \& x \notin B^{t}\right)\right\} .
$$

Then $Z$ is an r.e. set. We show that $A \cup\left(W_{e}-B\right)={ }^{*} A \cup\left(\left(W_{e}-E_{i}\right) \cap\right.$ $Z$ ), an r.e. set. Since $h^{*}(j)=\infty, W_{e}-B \subseteq Z$, so $\subseteq^{*}$ follows. Choose $s$ sufficiently large so that for all $t>s, b_{t}$ is not captured by any $r<j$ such that $r \in J$. Suppose that $x \in\left(W_{e}-E_{i}\right) \cap Z$. If $x \in B^{\prime}$, then $x \in W_{e}-B$. Otherwise, $x \in B$, so we may assume that $x=b_{t}$ for some $t>s$. Since $x \in Z, j$ potentially captures $x=b_{t}$ so $b_{t}$ must be captured by some $n<j$ such that $n \notin J$. By Lemma 2.12, $b_{t}=x \in A$. $\supseteq^{*}$ now follows.

The theorem is now immediate from Lemma 2.13 and Lemma 2.14.

Lachlan [2] described the requirements sufficient for the construction of an atomless $r$-maximal set which differs from the Robinson [11] construction. Norstad carried out the construction, and noted several interesting properties of the set constructed. We present Norstad's construction, and note additional properties of the set.

THEOREM 2.15. There exists an atomless $r$-maximal set $B$, and r.e. nonrecursive sets $B_{1}, B_{2}$ partitioning $B$ such that for all r.e. sets $W$, if $B^{\prime} \subseteq W$ and $W \cap B^{\prime} \neq * \varnothing$ then $B_{2} \cup(W-B)$ is r.e.

Proof. We will construct an r.e. set $\boldsymbol{H}_{0}$, and a sequence of pairwise disjoint infinite non-r.e. sets $\left\{K_{i}: i<\omega\right\}$ such that for all $i, H_{0} \cap K_{i}=\varnothing$ and $\cup\left\{K_{i}\right.$ : $i<\omega\} \cup H_{0}=\omega$. The sets $T_{i}=H_{0} \cup \cup\left\{K_{j}: j \leqslant i\right\}$ will form a tower of r.e. sets such that for every r.e. set $W$, either $H_{0}^{\prime} \subseteq^{*} W$ or $W \subseteq T_{i}$ for some $i$. It will then easily follow that $H_{0}$ is atomless and $r$-maximal. $B, B_{1}$, and $B_{2}$ will be defined from the tower.

Let $\left\{\left\langle x_{i}, y_{i}\right\rangle: i<\omega\right\}$ be a one-one recursive enumeration of $\omega^{2}$. During the construction, we will define a partial recursive function with recursive domain $d: \omega^{3} \rightarrow \omega$ such that for each $s, \lambda x y d(x, y, s)$ has domain $\subseteq\{i: i<s\}^{2}$. Let

$$
H_{0}=\{x<s:(\forall j)(\forall y)(x \neq d(j, y, s))\} .
$$


$d$ will be defined to maximize $e$-states of the elements of $H_{0}^{\prime}$ with respect to an appropriate $e$-state function $E$. Let

$$
\begin{aligned}
& \gamma(e, x, s) \\
& \quad=\left\{j<e:(\exists r \leqslant s)\left(x \in W_{j}^{r} \&(\forall y)(\forall k)(k<j \rightarrow x \neq d(k, y, r))\right)\right\},
\end{aligned}
$$

and

$$
E(e, x, s)=\sum\left\{2^{e-j}: j \in \gamma(e, x, s)\right\}
$$

By the bound given for the domain of $d$, it is evident that $\gamma$ and $E$ are recursive.

We say that $e$ requires attention at stage $s$ if either

(2.5) $d\left(x_{e}, y_{e}, s\right)$ is defined and there is a $v \leqslant s$ such that $v \notin H_{0}^{s}, v \neq$ $d\left(x_{i}, y_{i}, s\right)$ for all $i<e$, and $E\left(e, d\left(x_{e}, y_{e}, s\right), s\right)<E(e, v, s)$; or

(2.6) $d\left(x_{e}, y_{e}, s\right)$ is undefined.

The construction. Let $e$ be the least number which requires attention at stage $s$. If (2.5) holds for $e$ and $s$, let $v(s)$ be the least $v$ satisfying (2.5) for $e$ and $s$, and let $v(s)=s$ otherwise. Define

$$
d\left(x_{i}, y_{i}, s+1\right)= \begin{cases}d\left(x_{i}, y_{i}, s\right) & \text { if } i<e, \\ v(s) & \text { if } i=e, \\ \text { undefined } & \text { if } i>e .\end{cases}
$$

This completes the construction. Let $H_{0}=\cup_{s} H_{0}^{s}$. Clearly $H_{0}$ is r.e.

LEMMA 2.16. For all $e<\omega, \lim _{s} d\left(x_{e}, y_{e}, s\right)<\infty$.

Proof. We proceed by induction on $e$. Suppose that the lemma holds for all $j<e$. Choose $s$ sufficiently large so that for all $t \geqslant s$ and $j<e, d\left(x_{j}, y_{j}, s\right)$ $=d\left(x_{j}, y_{j}, t\right)$. Then for all $t>s, d\left(x_{e}, y_{e}, t\right)$ is defined. If $t>s$ and $d\left(x_{e}, y_{e}, t\right.$ $+1) \neq d\left(x_{e}, y_{e}, t\right)$, then $e$ requires attention at stage $t$ and $E\left(e, d\left(x_{e}, y_{e}, t\right), t\right)$ $<E\left(e, d\left(x_{e}, y_{e}, t+1\right), t\right)$. As $E(e, x, s)<2^{e+1}$ for all $e$ and $x$, and as whenever $u<v$ then $E(e, x, u)<E(e, x, v)$ for all $x$, only finitely many such stages $t$ can exist. Hence $\lim _{t} d\left(x_{e}, y_{e}, t\right)<\infty$.

By Lemma 2.16, the function $d^{*}\left(x_{e}, y_{e}\right)=\lim _{s} d\left(x_{e}, y_{e}, s\right)$ is total. $d^{*}$ is clearly one-one, as $\lambda x y d(x, y, s)$ is one-one for all $s<\omega$.

LEMMA 2.17. For all $e<\omega, \lim _{j} \lim _{s} E\left(e, d^{*}\left(x_{j}, y_{j}\right), s\right)<\infty$.

Proof. From the proof of Lemma 2.16, it follows that for all $e, j<\omega$ $E^{+}(e, j)=\lim _{s} E\left(e, d^{*}\left(x_{j}, y_{j}\right), s\right)<\infty$. Fix $e<\omega$. Suppose that $e<u<v$. Choose $s$ sufficiently large so that for all $t>s, d\left(x_{u}, y_{u}, t\right)=d^{*}\left(x_{u}, y_{u}\right)$, $d\left(x_{v}, y_{v}, t\right)=d^{*}\left(x_{v}, y_{v}\right), E^{+}(e, u)=E\left(e, d^{*}\left(x_{u}, y_{u}\right), t\right)$ and $E^{+}(e, v)=$ $E\left(e, d^{*}\left(x_{v}, y_{v}\right), t\right)$. Then $v$ cannot require attention at stage $s+1$, hence $E^{+}(e, u)>E^{+}(e, v)$. As $E^{+}(e, x)<2^{e+1}$ for all $x$, we must have $E^{+}(e, u)$ $=E^{+}(e, v)$ for all sufficiently large $u, v$. Hence $\lim _{u} E^{+}(e, u)<\infty$.

By Lemma $2.17, E^{*}(e)=\lim _{u} \lim _{s} E\left(e, d^{*}\left(x_{u}, y_{u}\right), s\right)$ is well defined.

For all $e>0$, let $K_{e}=\left\{x:(\exists y)\left(x=d^{*}(e, y)\right)\right\}$ and

$$
C_{e}=\left\{x:(\exists s)(\exists y)\left(x \in H_{0}^{s+1} \& x=d(e, y, s) \& E(e, x, s)=E^{*}(e)\right)\right\} \text {. }
$$


LEMMA 2.18. For all $e<\omega, C_{e} \cup K_{e}$ is r.e.

Proof. Fix $e<\omega$. Given $x<\omega$, search for the least $s$ such that either for some $y, x=d(e, y, s)=d(e, y, s+1)$ and $E(e, x, s)=E^{*}(e)$, or $x \in H_{0}^{s+1}$. By Lemma 2.17 and the definition of $E$, if no such $s$ exists then $x \notin C_{e} \cup K_{e}$ for all but finitely many $x$. Assume that $s$ exists, and fix $s$. If $x \in H_{0}^{s+1}$, then $x \in C_{e} \cup K_{e}$ if and only if $x=d(e, y, s)$ for some $y$ and $E(e, x, s)=E^{*}(e)$. Otherwise, $x=d(e, y, s)=d(e, y, s+1)$ and $E(e, x, s)=E^{*}(e)$. As $\{z$ : $\left.(\exists t)\left(E(e, z, t)>E^{*}(e)\right)\right\}$ is finite, by Lemma 2.17 we may assume that $\lim _{t}(E(e, x, t))=E^{*}(e)$. We claim under this assumption that $x \in C_{e} \cup K_{e}$. Suppose that $x \notin K_{e}$. Then there is a least $t>s$ such that $x \neq d(e, y, t+1)$. Fix such a $t$. If $x \in H_{0}^{t+1}$, then $x \in C_{e}$. Assume that $x \notin H_{0}^{t+1}$ for the sake of obtaining a contradiction. Then there is some $k<j$ such that $x=$ $d\left(x_{k}, y_{k}, t+1\right)$, where $\langle e, y\rangle=\left\langle x_{j}, y_{j}\right\rangle$. Hence $\left.E(k, x, t)\right\rangle$ $E\left(k, d\left(x_{k}, y_{k}, t\right), t\right)$. Furthermore, $d\left(x_{k}, y_{k}, t-1\right)=d\left(x_{k}, y_{k}, t\right)$ and

$$
E(k, x, t-1)<E\left(k, d\left(x_{k}, y_{k}, t-1\right), t-1\right),
$$

else some $r<k$ would require attention at stage $t-1$, and $d\left(x_{j}, y_{j}, t\right)$ would not be defined. Thus we must have $E(k, x, t)>E(k, x, t-1)$. Hence for some $m<k, x \in W_{m}^{t}$ and for all $u<\omega$ and $p<m, x \neq d(p, u, t)$; by the definition of $\gamma(e, x, s)$ we must therefore have $m<e$. But then $E(e, x, t)>$ $E(e, x, t-1)>E^{*}(e)$, a contradiction.

LEMMA 2.19. For all $e<\omega$, either $W_{e} \subseteq^{*} H_{0} \cup \cup\left\{K_{i}: i<e\right\}$ or $H_{0}^{\prime} \subseteq^{*} W_{e}$.

Proof. If $E^{*}(e)$ is odd, then by Lemma 2.17 and the definition of $E$, $d^{*}\left(x_{i}, y_{i}\right) \in W_{e}$ for all but finitely many $e$. Hence $H_{0}^{\prime} \subseteq{ }^{*} W_{e}$. Suppose that $E^{*}(e)$ is even. Fix $\langle k, v\rangle$ such that $k>e$. By Lemma 2.17 , for all but finitely many such $\langle k, v\rangle, \lim _{s} E\left(e, d^{*}(k, v), s\right)=E^{*}(e)$, so by the definition of $\gamma(e, x, s), d^{*}(k, v) \notin W_{e}$. Hence $W_{e} \subseteq{ }^{*} H_{0} \cup \cup\left\{K_{i}: i<e\right\}$.

For all $e<\omega$, let $D_{e}=\left\{x:(\exists s)(\exists y)\left(x=d(e, y, s) \& x \in H_{0}^{s+1}\right)\right\}$. Clearly $C_{e} \subseteq D_{e}$. Let $B=H_{0} \cup K_{0}, B_{2}=\bigcup\left\{D_{e}: e>0\right\}$ and $B_{1}=B-B_{2}$. Then $B_{1}$ and $B_{2}$ partition $B$. As $C_{0} \subseteq H_{0}$, by Lemma $2.18, B$ is r.e. $B_{2}$ is seen to be r.e. from its definition. $B_{1} \cap H_{0}$ is r.e. and $C_{0} \subseteq B_{1}$. But $B_{1}=\left(B_{1} \cap H_{0}\right) \cup$ $\left(C_{0} \cup K_{0}\right)$, so $B_{1}$ is r.e. by Lemma 2.18. Since $H_{0}^{\prime} \nsubseteq{ }^{*} B_{1}^{\prime}, B_{1}$ cannot be recursive, else by Lemma 2.19 ,

$$
\omega=B_{1} \cup B_{1}^{\prime} \subseteq{ }^{*} H_{0} \cup \bigcup\left\{K_{i}: i<e\right\}
$$

for some $e<\omega$, but this latter set is co-infinite.

Let $R$ be any recursive set. Assume that $R \cap B^{\prime}$ and $R^{\prime} \cap B^{\prime}$ are both infinite for the sake of obtaining a contradiction. Then there are $m, n<\omega$ such that $R \cap B^{\prime}=W_{m}$ and $R^{\prime} \cap B^{\prime}=W_{n}$. As $B^{\prime} \subseteq H_{0}^{\prime}$, we must have $H_{0}^{\prime} \nsubseteq^{*} W_{n}$ and $H_{0}^{\prime} \nsubseteq^{*} W_{m}$. Let $k=\max \{m, n\}$. By Lemma 2.19, $B^{\prime} \subseteq W_{m} \cup$ $W_{n} \subseteq{ }^{*} H_{0} \cup \cup\left\{K_{i}: i<k\right\}$. But $K_{k+1}$ is infinite, $K_{k+1} \subseteq B^{\prime}$, and $K_{k+1} \cap$ $\left(H_{0} \cup \cup\left\{K_{i}: i<k\right\}\right)=\varnothing$, a contradiction. It now follows that $B$ is $r$-maximal. 
Suppose that $B_{2}$ is recursive for the sake of obtaining a contradiction. Then $B_{2}^{\prime}=W_{e}$ for some $e<\omega$. As $C_{1} \subseteq B_{2}$, by Lemmas 2.16 and $2.18, K_{1}=W_{e} \cap$ $\left(C_{1} \cup K_{1}\right)$ is an infinite r.e. subset of the $r$-cohesive set $B^{\prime}$, contradicting Remark 2.10. Hence $B_{2}$ is not recursive.

Suppose that $M=W_{e}$ is a maximal superset of $B$ for the sake of obtaining a contradiction. Then $H_{0}^{\prime} \nsubseteq^{*} W_{e}$, so by Lemma $2.19, M \subseteq \subseteq^{*} H_{0} \cup \cup\left\{K_{i}\right.$ : $i<e\}$. By Lemma 2.18, $C_{e+1} \cup K_{e+1}$ is an r.e. set such that both it and its complement have infinite intersection with $M^{\prime}$, contradicting the maximality of $M$. Hence $B$ is atomless.

Suppose that $B^{\prime} \nsubseteq^{*} W_{e}$ and $W_{e} \cap B^{\prime} \neq \neq^{*} \varnothing$. By Lemma 2.19, $W_{e} \subseteq{ }^{*} H_{0}$ $\cup \cup\left\{K_{i}: i<e\right\}$. Hence

$$
\begin{aligned}
B_{2} \cup\left(W_{e}-B\right) & ={ }^{*} B_{2} \cup\left(\bigcup\left\{K_{i}: 1<i \leqslant e\right\} \cap W_{e}\right) \\
& ={ }^{*} B_{2} \cup\left(\bigcup\left\{C_{i} \cup K_{i}: 1<i<e\right\} \cap W_{e}\right)
\end{aligned}
$$

an r.e. set by Lemma 2.18 . This completes the proof of the theorem.

Let $A$ be an r.e. nonrecursive set. We say that $A$ is almost recursive if for every recursive set $R$, either $R \cap A$ or $R^{\prime} \cap A$ is recursive. The following facts about almost recursive sets can easily be verified by the reader.

RemarK 2.20. Let $A$ be an almost recursive set. Then

(2.7) if $A$ is simple, then $A$ is $r$-maximal;

(2.8) if $A_{1}$ and $A_{2}$ are a splitting of $A$, then $A_{1}$ and $A_{2}$ are almost recursive;

(2.9) if $B$ is a major subset of $A$, then $B$ is almost recursive.

Let $A \subseteq B$ be r.e. nonrecursive sets such that neither $A \cup B^{\prime}$ nor $B-A$ is r.e. $A$ is said to be an adequate subset of $B$ if for every r.e. set $W \subseteq B$, if $B-A \subseteq W$ then there is a recursive set $R$ such that $B-A \subseteq R$ and $W \cup(R-B)$ is r.e. Note that if $A$ is a small subset of $B$, then $A$ is an adequate subset of $B$. Remarks following the proof of Theorem 2.21 show that adequacy does not imply smallness.

In our next theorem, we construct several r.e. sets, among which are $H_{1}$ and $K$, such that $K$ is a small subset of $H_{1}$. The usual requirements to make $K$ a small subset of $H_{1}$ clash with other requirements, and we were unable to use them. Instead, we arrange the construction to make $H_{1}$ an almost recursive set and $K$ an adequate subset of $H_{1}$. These two properties are equivalent to making $K$ a small subset of $H_{1}$ given other properties of the sets constructed. Remark 2.34 will explicitly give these properties. The theorem extends results of Lachlan [4] and Lerman, Shore, and Soare [7].

TheOREM 2.21. There exist an atomless hhs set $\mathrm{H}$, a splitting $\mathrm{H}_{1}, \mathrm{H}_{2}$ of $\mathrm{H}$, and an r.e. subset $K$ of $H$ such that $K$ is a small r-maximal major subset of $H_{1}$ and for any r.e. set $W$, if $W-H_{2}$ is r.e. then $W-H$ is r.e.

Proof. By Theorem 2.9, fix an atomless hhs set $H$ with a $\Delta_{3}^{0}$ preference function. Fix a one-one recursive enumeration $\left\{b_{s}: s<\omega\right\}$ of $H$ and let $H^{s}=\left\{b_{i}: i<s\right\}$. Let $h^{*}: \omega \rightarrow\{0,1\}$ be a $\Delta_{3}^{0}$ preference function for $H$. Fix a 
recursive function $h: \omega^{3} \rightarrow \omega$ such that for all $x<\omega, h^{*}(x)=$ $\lim _{t} \lim _{s} h(t, s, x)$. For all $t, x<\omega$, let $h^{+}(t, x)=\lim _{s} h(t, s, x)$.

We will construct $H_{1}, H_{2}$, and $K$ by stages $\{s: s<\omega\} . H_{1}^{s}, H_{2}^{s}$ and $K^{s}$ will be sets of elements placed in $H_{1}, H_{2}$, and $K$ respectively before stage $s$. We will have a partial recursive function with recursive domain $d: \omega^{2} \rightarrow \omega$ such that for each $s, \lambda i d(s, i)$ will be one-one with range $H_{1}^{s}-K^{s} . d^{*}(i)=$ $\lim _{s} d(s, i)$ will be total with range $H_{1}-K$. Let $\left\{R_{e}^{i}: e<\omega \& i \leqslant 1\right\}$ be the enumeration of pairs of recursive sets mentioned earlier.

Many of the properties we desire of our sets will follow from the construction without specifically having to force these properties to hold. We will only take care to guarantee that $H_{1}$ and $H_{2}$ partition $H$ and that $K$ is an adequate $r$-maximal subset of $H_{1}$.

In order to guarantee that $K$ is an adequate subset of $H_{1}$, we will enumerate r.e.-sets $\left\{T_{e}: e<\omega\right\}$ during the course of the construction. $T_{e}$ will be used in conjunction with the appropriate recursive set $R$ to show that $W_{e} \cup\left(R-H_{1}\right)$ is r.e. whenever necessary. The definition of $T_{e}$ enables us to obtain an r.e. subset $T$ of $T_{e}$ such that $R-H_{1}=^{*} T \cap H_{1}^{\prime}$ and $T \subseteq{ }^{*} W_{e} \cup\left(R-H_{1}\right)$. We say that $x$ is protected by $e$ at stage $s$ if $x \in\left(H_{1}^{s+1} \cap T_{e}^{s}\right)-\left(K^{s}-W_{e}^{s}\right)$. In order to guarantee that $H_{1}-K$ is $r$-cohesive, we will try to maximize $e$-states of elements of $H_{1}-K$, whenever the adequacy conditions permit. Let

$$
\gamma(e, t, x, s)=\left\{i \leqslant e: x \in R_{i, s}^{h(t, s, i)}\right\}
$$

and

$$
E(e, t, x, s)=\sum\left\{2^{e-i}: i \in \gamma(e, t, x, s)\right\}
$$

We say that $e$ requires attention at stage $s$ if $d(s, e)$ is not protected by any $i<e$ at stage $s$, and either

(2.10) for some $n>e, E(e, e, d(s, e), s)<E(e, e, d(s, n), s)$; or

(2.11) $E(e, e, d(s, e), s)<E\left(e, e, b_{s}, s\right), d(s, e)<b_{s}$, and for all $t$ such that $b_{s}<t<s$, and all $i<e, h(e, t, i)=h(e, s, i)$ and $d(s, e)=d(t, e)$; or

(2.12) $d(s, e)$ is undefined.

The construction.

Stage $s$. Let $e$ be the least number which requires attention at stage $s$. Choose the first of (2.10), (2.11) and (2.12) satisfied by $e$ at stage $s$, and adopt the appropriate case below.

Case 1. (2.10) is chosen. Place $b_{s} \in H_{2}$ and $d(s, e) \in K$. Define

$$
d(s+1, i)= \begin{cases}d(s, i) & \text { if } i \neq e, n \text { and } d(s, i) \text { is defined, } \\ d(s, n) & \text { if } i=e, \\ \text { undefined } & \text { otherwise. }\end{cases}
$$

Case 2. (2.11) is chosen. Place $b_{s} \in H_{1}$ and $d(s, e) \in K$. Define

$$
d(s+1, i)= \begin{cases}d(s, i) & \text { if } i \neq e \text { and } d(s, i) \text { is defined } \\ b_{s} & \text { if } i=e \\ \text { undefined } & \text { otherwise }\end{cases}
$$


Case 3. (2.12) is chosen. If $b_{s}<s$, place $b_{s} \in H_{2}$, and define

$$
d(s+1, i)= \begin{cases}d(s, i) & \text { if } d(s, i) \text { is defined, } \\ \text { undefined } & \text { otherwise. }\end{cases}
$$

If $b_{s}>s$, place $b_{s} \in H_{1}$, and define

$$
d(s+1, i)= \begin{cases}d(s, i) & \text { if } d(s, i) \text { is defined, } \\ b_{s} & \text { if } i=e, \\ \text { undefined } & \text { otherwise. }\end{cases}
$$

In all cases, for each $i \leqslant s$, let $N(s, i)$ be the least $m<s$ such that $\left.H_{1}^{s+1}\right|_{m}-\left.\left.\left.K^{s+1}\right|_{m} \subseteq W_{i}^{s}\right|_{m} \subseteq H_{1}^{s+1}\right|_{m}$. Enumerate in $T_{i}^{s+1}$ all elements of $H^{\prime s+1} \mid N(s, i)$.

This completes the construction. For all $e<\omega$, let $T_{e}=\cup_{s} T_{e}^{s}$.

LEMMA 2.22. $d^{*}$ is total and one-one. Furthermore, $\operatorname{rng}\left(d^{*}\right)=H_{1}-K$.

Proof. We proceed by induction on $e$. Assume that $d^{*}(i)$ is defined for all $i<e$. Fix $s$ sufficiently large so that for all $t>s$ and $i<e, d(t, i)=d^{*}(i)$, and for all $t>s$ and $i<e, h(e, t, i)=h^{+}(e, i)$. As $\left\{s: b_{s}>s\right\}$ is infinite, there must be a least $r>s$ such that $d(r, e)$ is defined. Then for all $t>r$, $d(t, e)$ is defined. If $t>s$ and $d(t, e) \neq d(t+1, e)$, then $e$ requires attention at stage $t$ and $E(e, e, d(t, e), t)<E(e, e, d(t+1, e), t)$. By choice of $r>s$, for all $v, u>r$ and $x<\omega$, if $u<v$ then $E(e, e, x, u)<E(e, e, x, v)$. As $E(e$, $e, x, u)<2^{e+1}$ for all $x, u<\omega$, only finitely many such $t$ can exist. Hence $d^{*}(e)=\lim _{s} d(s, e)$ must exist. As $\lambda e d(s, e)$ is one-one for all $e, d^{*}$ must be one-one.

As $\operatorname{rng}(\lambda e d(s, e))=H_{1}^{s}-K^{s}$ for all $s$, we must have $\operatorname{rng}\left(d^{*}\right) \subseteq H_{1}-K$. If $x \in H_{1}-K$, then $x \in \operatorname{rng}(\lambda e d(s, e))$ for all sufficiently large $s$. Furthermore, if $t>s$ and $x=d(t, e)=d(s, n)$, then $e<n$. Hence $H_{1}-K \subseteq \operatorname{rng}\left(d^{*}\right)$.

LEMMA 2.23. For all $e$, there exists an $M(e)$ such that, for all $x>M(e)$ and all sufficiently large $s, x$ is not protected by $e$ at stage $s$.

Proof. We may assume that $H_{1}-K \subseteq W_{e}$, else only finitely many $x$ can ever be enumerated in $T_{e}$. If $x$ is ever protected by $e$, then $x \in T_{e} \cap H_{1}$. Any such $x$ must eventually appear either in $W_{e}$ or $K$, and can never be protected thereafter.

For each $e<\omega$, let $\gamma^{*}(e)=\left\{i<e: R_{i}^{0} \cup R_{i}^{1} \neq{ }^{*} \varnothing\right\}$ and $E^{*}(e)=\Sigma\left\{2^{e-i}\right.$ : $\left.i \in \gamma^{*}(e)\right\}$.

LEMMA 2.24. For all $e<\omega \lim _{x} \lim _{v} \lim _{s} E\left(e, v, d^{*}(x), s\right)=E^{*}(e)$.

Proof. For all $v, x, s<\omega, E(e, v, x, s)<2^{e+1}$. Furthermore, for all $v<\omega$ there is an $s$ such that for all $x<\omega$ and all $r>t>s, E(e, v, x, t)<$ $E(e, v, x, r)$. As $\lim _{t} \lim _{s} h(t, s, e)=h^{*}(e)$, we must have $E^{+}(e, x)=$ $\lim _{v} \lim _{s} E(e, v, x, s)$ existing for all $e, x<\omega$.

We proceed by induction on $e$. Assume that the lemma holds for all $i<e$. We assume as an induction hypothesis that for all $j<e$, if $j \in \gamma^{*}(j)$, then

$$
d^{*}(x) \in R_{i}^{h(i)} \text { for all sufficiently large } x .
$$


Note that if $j<e$, then $\gamma^{*}(j)=\gamma^{*}(e) \mid j+1$, so (2.13) holds for all $j \in$ $\gamma^{*}(e) \mid e$.

First assume that $R_{e}^{0} \cup R_{e}^{1}=^{*} \varnothing$. Then $e \notin \gamma^{*}(e)$, and for only finitely many $x$ can $x \in R_{i}^{h^{*}(i)}$. Hence for only finitely many $x$ can $E^{+}(e, x)$ be odd. By (2.13), we see that $E^{+}\left(e, d^{*}(x)\right)=E^{*}(e)$ for all sufficiently large $x$.

Now assume that $R_{e}^{0} \cup R_{e}^{1} \neq * \varnothing$. Let

$$
J=\left\{x:(\exists s)\left(\forall j \in \gamma^{*}(e)\right)\left(x \in R_{j, s}^{h^{*}(j)}-H^{s}\right)\right\} .
$$

Since $h^{*}$ is a preference function for $H, J \cap H^{\prime}$ must be infinite. As $J$ is r.e. and $H$ is simple, $J \cap H \neq{ }^{*} \varnothing$. By (2.13) and Lemma 2.23, for all sufficiently large $x, E^{*}(e)-1<E^{+}\left(e, d^{*}(x)\right)<E^{*}(e)$ and $d^{*}(x)$ is not protected by any $i<e$ at all sufficiently large stages. Assume that $E^{+}\left(e, d^{*}(x)\right)=E^{*}(e)-1$ for infinitely many $x$, for the sake of obtaining a contradiction. Fix such an $x$ for which $h^{+}(x, e)=h^{*}(e)$ and $x>e$. Choose $s$ sufficiently large so that for all $t>s, d(t, x)=d^{*}(x), h(x, t, e)=h^{*}(e)$, and $d^{*}(x)$ is not protected by any $i<e$ at stage $t$. Let $t>s$ be the least stage such that $b_{t} \in J$ and $b_{t}>\max (s, x)$. Then $E\left(e, x, b_{t}, t\right)=E^{*}(e)$ and $E\left(e, x, d^{*}(x), t\right)<E^{*}(e)$. As $x>e, E\left(x, x, b_{t}, t\right)>E\left(x, x, d^{*}(x), t\right)$, so $x$ requires attention via (2.11) at stage $t$. But this is impossible, else $d^{*}(x)=d(t, x) \neq d(t+1, x)$ contradicting the choice of $x$. Hence $E^{+}\left(e, d^{*}(x)\right)=E^{*}(e)$, and (2.13) holds for $i=e$.

LEMMA 2.25. $K$ is an r-maximal subset of $H_{1}$.

Proof. Let $R$ be any recursive set. Fix the least $e$ such that $R=R_{e}^{0}$ and $R^{\prime}=R_{e}^{1}$, and let $E^{+}$be as in Lemma 2.24. Then by Lemma 2.24, as $e \in \gamma^{*}(e), E^{+}\left(e, d^{*}(x)\right)=E^{*}(e)$ for all sufficiently large $x$. Hence for all but finitely many $x, d^{*}(x) \in R_{e}^{h^{*}(e)}$. Thus $H_{1}-K$ is $r$-cohesive.

LEMMA 2.26. $K$ is a major subset of $H_{1}$.

Proor. It suffices to show that $\mathrm{K} \cup \mathrm{H}_{2}$ is a major subset of $\mathrm{H}$, as $\mathrm{H}_{1}, \mathrm{H}_{2}$ partition $H$. Let $W$ be an r.e. set such that $H^{\prime} \subseteq W$. By Theorem 2.1, there is a recursive set $R$ such that $R \subseteq W$ and $R^{\prime} \subseteq H$, so $H^{\prime} \subseteq R$. It now suffices to show that $H_{1}-K \subseteq \subseteq^{*} R$. Let $R=R_{e}^{0}, R^{\prime}=R_{e}^{1}$. Then $h^{*}(e)=0$. By Lemma 2.24, we must have $E^{+}\left(e, d^{*}(x)\right)=E^{*}(e)$ for all sufficiently large $x$, so for all but finitely many $x, d^{*}(x) \in R_{e}^{h^{*}(e)}=R_{e}^{0}=R$. Hence by Lemma 2.22, $H_{1}-K \subseteq R$.

LEMMA 2.27. $K$ is an adequate subset of $H_{1}$.

Proof. Suppose that $H_{1}-K \subseteq W_{e} \subseteq H_{1}$. Let $R=\cap\left\{R_{i}^{h^{*}(i)}: i \in \gamma^{*}(e)\right\}$ and $R^{s}=\bigcap\left\{R_{i, s}^{h^{*}(i)}: i \in \gamma^{*}(e)\right\}$ for all $s<\omega$. As in the proof of Lemma 2.25, we note that $H_{1}-K \subseteq \subseteq^{*} R$. We will show that $W_{e} \cup\left(R-H_{1}\right)$ is r.e.

Since $H_{1}-K \subseteq W_{e} \subseteq H_{1}$, we must have $H_{1}^{\prime} \subseteq T_{e}$. Let $I=\{x:(\exists s)(x \in$ $\left.\left.R^{s} \cap T_{e}^{s} \& x \notin H^{s}\right)\right\}$. Then $I-H_{1}=R-H_{1}$, so it suffices to show that $W_{e} \cup\left(I-H_{1}\right)$ is r.e. This will follow once we show that $I \cap H_{1} \subseteq W_{e}$.

Suppose that $x \in I \cap H_{1}$. By Lemma 2.22, $V=\{y:(\exists s)(\exists i)(i<e$ \& $y=$ $d(s, i))\}$ is finite, so we may assume without loss of generality that $x \notin V$. By 
Lemma 2.23, we may assume without loss of generality that $x>M(e)$.

Let $x=b_{s}$. Without loss of generality, we may assume that $d(s, i)=d^{*}(i)$ for all $i<e$. As $x \in H_{1}, x=d(s+1, i)$ for some $i>e$. Then for all $t>s, x$ cannot be placed in $K^{t+1}$ if $x$ is protected by $e$ at stage $t$. Since $x \in H_{1}^{s+1} \cap$ $T_{e}^{s}$, there must be a least stage $t>s$ such that $x$ is not protected by $e$ at stage $t$. But then $x \in W_{e}^{t} \subseteq W_{e}$. Hence $I \cap H_{1} \subseteq{ }^{*} W_{e}$.

LEMMA 2.28. $H_{1}$ and $H_{2}$ partition $H$, and $H_{1}$ is not recursive.

Proof. Clearly $H_{1}$ and $H_{2}$ partition $H$. If $H_{1}$ were recursive, then we would have $H^{\prime} \subseteq H_{1}^{\prime}$ but $\left(H_{1}-K\right) \cap H_{1}^{\prime}=\varnothing$ contradicting Lemma 2.26.

For all $e, s<\omega$, let $R_{e, s}=\bigcap\left\{R_{i, s}^{h^{*}(i)}: i \in \gamma^{*}(e)\right\}$ and $R_{e}=\cup_{s} R_{e, s^{*}}$

LEMMA 2.29. For all recursive sets $R$, if $R \cap H^{\prime} \neq * \varnothing$ then $R \cap H_{2} \neq \varnothing$.

Proof. Fix $e<\omega$ such that $R=R_{e}^{0}$ and $R^{\prime}=R_{e}^{1}$. Let $n<\omega$ be the least number such that $R-R_{n} \neq \neq^{*} \varnothing$. Such an $n$ must exist as $H$ is atomless hhs. Fix $M$ such that for all $x>M, E^{+}\left(n, d^{*}(x)\right)=E^{*}(n)$. Fix $s$ sufficiently large so that for all $t>s$ and $x<\max (M, n), d(t, x)=d^{*}(x)$. Let $V^{r}=\{y<r$ : $\left.y \notin H^{r} \& y \in R_{e, r}^{0} \&(\forall u)\left(d(r, u)<y \rightarrow E(n, u, d(r, u), r)=E^{*}(n)\right)\right\}$. Let $V=U_{r} V^{r} . V \subseteq R_{e}^{0}$ is clearly r.e. and $V \cap H^{\prime}=R_{e}^{0} \cap H^{\prime}$. Furthermore, $V-R_{n}$ is r.e. and $\left(V-R_{n}\right) \cap H^{\prime} \neq * \varnothing$. As $H^{\prime}$ is immune, $\left(V-R_{n}\right) \cap$ $H \neq \varnothing^{*} \varnothing$. Let $x=b_{t} \in\left(V-R_{n}\right) \cap H$ with $t>s$. Then $x \in V^{r}$ for some $r<t$. If Case 1 is followed at stage $t$, then $x \in H_{2}$ so $V \cap H_{2} \subseteq R_{e}^{0} \cap H_{2} \neq$ $\varnothing$. Case 2 cannot be followed at stage $t$, because if $x=d(t+1, i)$, then $d(t, i)<x$ and $i>n$ so $E(n, i, d(t, i), t)=E^{*}(n)>E(n, i, x, t)$ so $E(i$, $i, x, t)<E(i, i, d(t, i), t)$ and thus the first clause of (2.11) fails for $x=b_{t}$. If Case 3 is followed at stage $t, x<t$ so $x \in H_{2}$ and again we have $H_{2} \cap R_{e}^{0} \neq$ $\varnothing$.

LEMMA 2.30. $\mathrm{H}_{2}$ is not recursive.

Proof. Suppose that $\mathrm{H}_{2}$ is recursive for the sake of obtaining a contradiction. Let $H_{2}^{\prime}=R_{e}^{0}, H_{2}=R_{e}^{1}$. Then $R_{e} \subseteq H_{2}^{\prime}=R_{e}^{0}$ so by Lemma 2.29, $R_{e} \cap H_{2} \neq \varnothing$; hence $H_{2} \cap H_{2}^{\prime} \neq \varnothing$, a contradiction.

LEMMA 2.31. For all r.e. sets $W$, if $W-H_{2}$ is r.e. then $W-H$ is r.e.

Proof. Let $W-H_{2}$ be r.e. We cannot have $W \cap H^{\prime} \neq \neq^{*} \varnothing$, else as $H$ is hhs, $H \cup W^{\prime}$ is r.e., so by Theorem 2.1 there is a recursive set $R \subseteq W-H_{2}$ with $R \cap H^{\prime}=W \cap H^{\prime}$; by Lemma $2.29, R \cap H_{2} \neq \varnothing$ yielding a contradiction. Hence $W \subseteq \subseteq^{*} H$ and the lemma is immediate.

LEMMA 2.32. $\mathrm{H}_{1}$ is almost recursive.

Proof. Let $R$ be a recursive set. We may assume that $R \cap H_{1}$ and $R^{\prime} \cap H_{1}$ are both infinite, else we are done. Fix $e$ such that $R=R_{e}^{0}$ and $R^{\prime}=R_{e}^{1}$. Without loss of generality, we may assume that $h^{*}(e)=0$. We will show that $R^{\prime} \cap H_{1}$ is recursive.

Let $x \in R_{e, s}^{1}$. If $x \in H^{s}$, then we can immediately decide whether or not 
$x \in H_{1}$. So assume that $x \notin H^{s}$. Fix $M$ such that for all $x>M$, $E^{+}\left(e, d^{*}(x)\right)=E^{*}(e)$ and $E^{+}(e, x)<E^{*}(e)$. Fix $t$ sufficiently large so that for all $r>t$ and $x \leqslant \operatorname{Max}(M, e), d(r, x)=d^{*}(x)$. Without loss of generality we may assume that $s>t$. Find the least stage $r>\max (x, t)$ such that for all $y<x$, if $y=d(r, i)$ then $E(e, i, d(r, i), r)=E^{*}(e)$ or $i<\operatorname{Max}(M, e)$. Clearly such a stage must exist. If $x \in H^{r}$, then we can decide whether or not $x \in H_{1}$. Suppose $x \notin H^{r}$. We claim then that $x \notin H_{1}$. For suppose $x$ is placed in $H_{1}$ at stage $u>r$. As $x<r<u$ Case 1 or Case 3 cannot be followed at stage $u$. If Case 2 is followed and $x=d(u+1, i)$, then $i>e$ and $d(u, i)<x$. Furthermore, by choice of $r$ and the conditions of Case 2 , $E(e, i, d(u, i), u)>E(e, i, d(u, i), r)=E^{*}(e)$ as $d(u, i)=d(r, i)$, and $E(e$, $i, x, u)<E^{*}(e)$ as $E^{+}(e, x)<E^{*}(e)$ and $x \notin R_{e}^{h^{*}(e)}$. Hence as $i>e, E(i$, $i, d(u, i), u)>E(i, i, x, u)$, so we cannot have $x=d(u+1, i)$. Hence Case 2 cannot be followed, and $x \notin H_{1}$. This procedure decides effectively whether or not $x \in R^{\prime} \cap H_{1}$, so $R^{\prime} \cap H_{1}$ is recursive.

LEMMA 2.33. $K$ is a small subset of $H_{1}$.

Proof. Let $W$ and $X$ be any r.e. sets such that $X \cap\left(H_{1}-K\right) \subseteq W$. We must show that $W \cup\left(X-H_{1}\right)$ is r.e. Since $H$ is hhs, $H \cup X^{\prime}$ is r.e. Since $X \cup\left(H \cup X^{\prime}\right)=\omega$, by the separation theorem, we can find a recursive set $R$ such that $R \subseteq X$ and $R^{\prime} \subseteq H \cup X^{\prime}$. Since $X-H_{1}=\left(H_{2} \cap X\right) \cup(R-$ $\left.H_{1}\right)$, it suffices to show that $W \cup\left(R-H_{1}\right)$ is r.e.

We first show that if $S$ is any recursive set such that $S \cap\left(H_{1}-K\right)={ }^{*} \varnothing$, then $S-H_{1}$ is recursive. Without loss of generality, we may assume that $S \cap\left(H_{1}-K\right)=\varnothing$. Since $S$ is recursive, we can prove $S-H_{1}$ recursive by proving that $S \cap H_{1}$ is recursive. By Lemma $2.32, H_{1}$ is almost recursive, so it suffices to show that $H_{1} \cap S^{\prime}$ is not recursive. But $H_{1} \cap S^{\prime}$ cannot be recursive else its complement would be an r.e. set containing $H_{1}^{\prime}$ and disjoint from $H_{1}-K$, contradicting the fact that $K$ is a major subset of $H_{1}$.

Since $H_{1}-K$ is $r$-cohesive, we need only consider the cases where $R \cap$ $\left(H_{1}-K\right)={ }^{*} \varnothing$ or $H_{1}-K \subseteq * R$. If $R \cap\left(H_{1}-K\right)=* \varnothing$, then by the previous paragraph, $R-H_{1}$ is recursive. Hence $W \cup\left(R-H_{1}\right)$ is r.e. If $H_{1}-K \subseteq * R$, then by Lemma 2.27 , there is a recursive set $T$ such that $H_{1}-K \subseteq T$ and $W \cup\left(T-H_{1}\right)$ is r.e. By the preceding paragraph, $T^{\prime}-$ $H_{1}$ is recursive. Hence

$$
W \cup\left(R-H_{1}\right)=W \cup\left(\left(\left(W \cup\left(T-H_{1}\right)\right) \cap R\right) \cup\left(\left(T^{\prime}-H_{1}\right) \cap R\right)\right)
$$

is seen to be r.e.

The theorem now follows from Lemmas 2.25, 2.26, 2.28, 2.30, 2.31 and 2.33.

Adequacy and almost recursiveness are equivalent to smallness under the conditions described in our next remark.

Remark 2.34. Let $H$ be an hhs set, $H_{1}$ an r.e. subset of $H$ such that $H-H_{1}$ is r.e., and let $K$ be an $r$-maximal major subset of $H_{1}$. Then $K$ is a small subset of $H_{1}$ if and only if $K$ is an adequate subset of $H_{1}$ and $H_{1}$ is almost recursive. 
Proof. The proof of Lemma 2.33 shows that $K$ is a small subset of $H_{1}$ if $K$ is an adequate subset of $H_{1}$ and $H_{1}$ is almost recursive. Assume that $K$ is a small subset of $H_{1}$. Then $K$ is easily seen to be an adequate subset of $H_{1}$. Let $R$ be any recursive set. Since $H_{1}-K$ is $r$-cohesive, we may assume without loss of generality that $R \cap\left(H_{1}-K\right)=\varnothing$. By smallness, $\varnothing \cup\left(R-H_{1}\right)=R$ $-H_{1}$ is r.e. Since $R-H_{1}$ and $R \cap H_{1}$ are r.e. sets partitioning $R, R \cap H_{1}$ must be recursive. Hence $H_{1}$ is almost recursive.

It is easily seen that smallness implies adequacy. The converse, however, is not true. One can easily modify the proof of Theorem 2.21 to show that if $H$ is any atomless hhs set with a $\Delta_{3}^{0}$ preference function, then $H$ has an adequate $r$-maximal major subset $K$. $K$, however, cannot be a small subset of $H$. For let $R$ be any recursive set such that $R \cap H^{\prime} \neq{ }^{*} \varnothing$ and $R^{\prime} \cap H^{\prime} \neq * \varnothing$. Since $K$ is an $r$-maximal subset of $H$, we can assume without loss of generality that $R \cap(H-K)=\varnothing$. Since $R \cap(H-K) \subseteq \varnothing$, if $K$ were a small subset of $H$, $\varnothing \cup(R-H)$ would be r.e. But this is impossible as $\varnothing \cup(R-H)$ is an immune set.

We also note that in Theorem 2.21, $H_{1}$ and $H_{2}$ cannot be a Friedberg splitting of $H . H_{1}$ and $H_{2}$ split $H$ and, by Lemma 2.31, $H_{2}$ satisfies (2.1). To see that $H_{1}$ does not satisfy (2.1), let $R$ be any recursive set such that $R \cap H^{\prime} \neq^{*} \varnothing$ and $R^{\prime} \cap H^{\prime} \neq \neq^{*} \varnothing$. As $H^{\prime}$ is immune, neither $R-H$ nor $R^{\prime}-H$ is r.e. Since $H_{1}$ is almost recursive, we can assume without loss of generality that $R \cap H_{1}$ is recursive. But then $R-H_{1}$ is the difference of two recursive sets, hence recursive, so (2.1) fails to hold.

3. Canonical realizations and necessary conditions. Let $(\mathscr{P}, \mathscr{B}) \subseteq(\mathcal{E}, \mathscr{Q})$ be a finite separated $d$-lattice with one component, and let $C$ be the complement of the outermost atom of $(\mathscr{P}, \mathscr{B})$, with $C$ non-hhs. Let $D$ and $E$ be r.e. sets partitioning $C$, and let $\left(\mathscr{P}_{1}, \mathscr{B}_{1}\right)=(\mathscr{P}, \mathscr{B}) \cap E$ and $\left(\mathscr{P}_{2}, \mathscr{B}_{2}\right)=(\mathscr{P}, \mathscr{B}) \cap$ $D$. We say that $\left(\mathscr{P}_{2}, \mathscr{B}_{2}\right)$ is a minimizer of $(\mathscr{P}, \mathscr{B})$ if

(3.1) there is an r.e. set $W$ such that $C \subset^{*} W \subset^{*} \omega, D \cup(W-C)$ is r.e., and $C \cup W^{\prime}$ is not r.e.; and

(3.2) for every pair of r.e. sets $D_{1}, E_{1}$ partitioning $C$ and every r.e. set $W$ such that $C \subset^{*} W \subset^{*} \omega, C \cup W^{\prime}$ is not r.e., and $D_{1} \cup(W-C)$ is r.e.,

(a) $(\mathscr{P}, \mathscr{B}) \cap D_{1}$ does not have smaller characteristic than $\left(\mathscr{P}_{2}, \mathscr{B}_{2}\right)$; and

(b) if $(\mathscr{P}, \mathscr{B}) \cap D_{1} \simeq\left(\mathscr{P}_{2}, \mathscr{B}_{2}\right)$ then $(\mathscr{P}, \mathscr{B}) \cap E_{1}$ does not have smaller characteristic than $\left(\mathscr{P}_{1}, \mathscr{B}_{1}\right)$.

If $\left(\mathscr{P}_{2}, \mathscr{B}_{2}\right)$ is a minimizer of $(\mathscr{P}, \mathscr{B})$, then $\left(\mathscr{P}_{1}, \mathscr{B}_{1}\right)$ is said to be the cominimizer of $(\mathscr{P}, \mathscr{B})$ associated with $\left(\mathscr{P}_{2}, \mathscr{B}_{2}\right)$. Note that if $\mathscr{P}=\{\varnothing, \omega, C\}$, then $\left(\mathscr{P}_{2}, \mathscr{B}_{2}\right)=\varnothing$ is a minimizer of $(\mathscr{P}, \mathscr{B})$ if and only if $C$ is not simple.

Let $(\mathscr{P}, \mathscr{B})$ have minimizer $\left(\mathscr{P}_{2}, \mathscr{B}_{2}\right)$ with associated cominimizer $\left(\mathscr{P}_{1}, \mathscr{B}_{1}\right)$. Then $\left(\mathscr{P}^{*}, \mathscr{B}^{*}\right)$ is said to be the minimizer refinement of $(\mathscr{P}, \mathscr{B})$ for $\left(\mathscr{P}_{2}, \mathscr{B}_{2}\right)$ if $\left(\mathscr{P}^{*}, \mathscr{B}^{*}\right)$ is the sub- $d$-lattice of $(\mathscr{E}, \mathscr{Q})$ generated by the elements of $\mathscr{P}_{1}$ and $\mathscr{P}_{2}$.

Let $(\mathscr{P}, \mathscr{B}) \subseteq(\mathcal{E}, \mathscr{Q})$ be a finite separated $d$-lattice with one component, and let $C$ be the complement of the outermost atom of $(\mathcal{P}, \mathscr{B})$, with $C$ not quasi-r-maximal. Let $D$ and $E$ be r.e. sets partitioning $C$ such that $E$ is not 
recursive and if $D$ is recursive then $D=\varnothing$, and let $\left(\mathscr{P}_{2}, \mathscr{B}_{2}\right)=(\mathscr{P}, \mathscr{B}) \cap D$ and $\left(\mathscr{P}_{1}, \mathscr{B}_{1}\right)=(\mathscr{P}, \mathscr{B}) \cap E$. We say that $\left(\mathscr{P}_{2}, \mathscr{B}_{2}\right)$ is a companion of $(\mathscr{P}, \mathscr{B})$ if $\left(\mathscr{P}_{1}, \mathscr{B}_{1}\right) \simeq(\mathscr{P}, \mathscr{B}) \cap C$ and there is a partition $\left\{R_{i}: i<\omega\right\}$ of $\omega$ consisting of recursive sets such that for all $i<\omega R_{i} \cap C^{\prime} \neq^{*} \varnothing,(\mathscr{P}, \mathscr{B}) \cap R_{0} \simeq$ $(\mathscr{P}, \mathscr{B})$, and $(\mathscr{P}, \mathscr{B}) \cap\left(R_{i} \cap C\right) \simeq\left(\mathscr{P}_{2}, \mathscr{B}_{2}\right)$ for all $i>1$.

Let $(\mathscr{P}, \mathscr{B})$ have companion $\left(\mathscr{P}_{2}, \mathscr{B}_{2}\right)=(\mathscr{P}, \mathscr{B}) \cap D$ and let $\left(\mathscr{P}_{1}, \mathscr{B}_{1}\right)=$ $(\mathscr{P}, \mathscr{B}) \cap E$ where $D$ and $E$ are as in the preceding paragraph. Then $\left(\mathscr{P}^{*}, \mathscr{B}^{*}\right)$ is said to be the companion refinement of $\left(\mathscr{P}, \mathscr{B}^{\prime}\right)$ for $\left(\mathscr{P}_{2}, \mathscr{B}_{2}\right)$ if $\left(\mathscr{P}^{*}, \mathscr{B}^{*}\right)$ is the sub- $d$-lattice of $(\mathscr{E}, \mathscr{Q})$ generated by the elements of $\mathscr{P}_{1}$ and $\mathscr{P}_{2}$.

For the rest of this section, fix a finite separated $d$-lattice $(L, A)$ with one component, having outermost atom $a_{0}$. We will construct three types of canonical realizations $(\mathscr{P}, \mathscr{B})$ of $(L, A)$ and isomorphisms $\psi:(\mathscr{P}, \mathscr{B}) \simeq$ $(L, A)$. The realization of type $\mathrm{i}$ will be defined in subcase $\mathrm{i}$ of the construction. The realization of type 1 will be the Lachlan canonical realization with $\psi\left(a_{0}^{\prime}\right)$ maximal. For each pair of lattices $\left(L_{1}, A_{1}\right)$ and $\left(L_{2}, A_{2}\right)$ which are embeddable in $(L, A) \mid a_{0}^{\prime}$ and whose fusion over $(L, A) \mid a_{0}^{\prime}$ under these embeddings is isomorphic to $(L, A) \mid a_{0}^{\prime}$ such that if $\left(L_{1}, A_{1}\right) \simeq(L, A) \mid a_{0}^{\prime}$ then $\left(L_{2}, A_{2}\right)$ $=\varnothing$, we will construct a canonical realization of type 2 , all of whose minimizers are isomorphic to $\left(L_{2}, A_{2}\right)$ and such that $\left(L_{1}, A_{1}\right)$ is isomorphic to all of its cominimizers. For each pair of $d$-lattices $\left(L_{1}, A_{1}\right)$ and $\left(L_{2}, A_{2}\right)$ such that $\left(L_{2}, A_{2}\right) \hookrightarrow\left(L_{1}, A_{1}\right) \simeq(L, A) \mid a_{0}^{\prime},\left(L_{2}, A_{2}\right) \neq \varnothing$, and $\left(L_{2}, A_{2}\right)$ is not isomorphic to the two-element boolean algebra, we construct a canonical realization of type 3, all of whose companions are isomorphic to $\left(L_{2}, A_{2}\right)$. In fact, instead of constructing $(\mathscr{P}, \mathscr{B})$, we construct a refinement $\left(\mathscr{P}^{\prime}, \mathscr{B}^{\prime}\right)$ of $(\mathscr{P}, \mathscr{B})$ such that if $(\mathscr{P}, \mathscr{B})$ is of type 1 then $\left(\mathscr{P}^{\prime}, \mathscr{B}^{\prime}\right)=(\mathscr{P}, \mathscr{B})$, if $(\mathscr{P}, \mathscr{B})$ is of type 2 then $\left(\mathscr{P}^{\prime}, \mathscr{B}^{\prime}\right)$ is a minimizer refinement of $(\mathscr{P}, \mathscr{B})$, and if $(\mathscr{P}, \mathscr{B})$ is of type 3 then $\left(\mathscr{P}^{\prime}, \mathscr{B}^{\prime}\right)$ is a companion refinement of $(\mathscr{P}, \mathscr{B})$.

$\psi$ will be defined on the atoms of $(L, A)$, and the definition will proceed by induction downwards, following the withinness relation of either $(L, A)$, $\left(L_{1}, A_{1}\right)$ or $\left(L_{2}, A_{2}\right)$.

The construction. Let $e$ be an atom of $(L, A)$ for type 1 realizations, and $e=a_{0}$ or $e$ an atom of $\left(L_{1}, A_{1}\right)$ or $\left(L_{2}, A_{2}\right)$ for type 2 or type 3 realizations. For any atom $c$, let $c^{+}$be the unique atom $a$ such that $c \prec_{j} a$ if $c \neq a_{0}$ and $c$ is not outermost in $\left(L_{1}, A_{1}\right)$ or $\left(L_{2}, A_{2}\right), c^{+}=a_{0}$ if $c$ is outermost in $\left(L_{1}, A_{1}\right)$ or $\left(L_{2}, A_{2}\right)$, and undefined if $c=a_{0}$. For any canonical generator $p$, let $p^{+}=L\left(A(p)^{+}\right)$. There is some ambiguity in this definition as an innermost atom is also a canonical generator. To avoid any confusion, we will use $a, b$, $c, d, e$ to denote atoms, and $p, q, r, s, t$ to denote canonical generators.

Assume that $\psi(e)$ has not yet been defined, but that $\psi\left(e^{+}\right)$has been defined. Let $e_{1}, \ldots, e_{k}$ be the atoms such that $e_{i}^{+}=e^{+}$for $1<i<k$, among which $e$ must occur. By induction, we may assume that none of $\psi\left(e_{1}\right), \ldots, \psi\left(e_{k}\right)$ has yet been defined.

Case 1. $e=a_{0}$. Define $\psi(L(e))=\omega$.

Subcase 1 . By Theorem 2.5 , let $M$ be a maximal r.e. set. Define $\psi(e)=M^{\prime}$. 
Subcase 2. Let $B$ be an atomless $r$-maximal set as in Theorem 2.15, partitioned by $B_{1}$ and $B_{2}$. If $\left(L_{2}, A_{2}\right) \neq \varnothing$, define $\psi(e)=B^{\prime}$, and if $\left(L_{2}, A_{2}\right)=$ $\varnothing$, define $\psi(e)=B_{1}^{\prime}$.

Subcase 3. Let $H$ be an atomless hhs set with a $\Delta_{3}^{0}$ preference function as in Theorem 2.9. Define $\psi(e)=H^{\prime}$.

Case 2. $e^{+}=a_{0}$.

Subcase 1. Let $E_{1}, \ldots, E_{k}$ be a Friedberg splitting of $M$ as in Theorem 2.4. Define $\psi\left(L\left(e_{i}\right)\right)=E_{i}$ for $1<i<k$. For each $i=1, \ldots, k$, let $C_{i}$ be a small major subset of $E_{i}$ as in Theorem 2.6: Define $\psi\left(e_{i}\right)=E_{i}-C_{i}$ if $e_{i}$ is not innermost, and $\psi\left(e_{i}\right)=E_{i}$ otherwise.

Subcase 2. If $\left(L_{2}, A_{2}\right)=\varnothing$, proceed as in Subcase 1 with $B_{1}$ replacing $M$. If $\left(L_{1}, A_{1}\right)=\varnothing$, proceed as in Subcase 1 with $B$ replacing $M$. If $\left(L_{1}, A_{1}\right) \neq \varnothing$ and $\left(L_{2}, A_{2}\right) \neq \varnothing$, we may assume that $\left\{e_{1}, \ldots, e_{k}\right\}$ is ordered so that $e_{i}$ is an atom of $\left(L_{2}, A_{2}\right)$ for $1<i<r$ and $e_{i}$ is an atom of $\left(L_{1}, A_{1}\right)$ for $r<i<k$. Let $E_{1}, \ldots, E_{r}$ be a Friedberg splitting of $B_{2}$ as in Theorem 2.4 and $E_{r+1}, \ldots, E_{k}$ a Friedberg splitting of $B_{1}$ as in Theorem 2.4. Define $\psi\left(L\left(e_{i}\right)\right)$ $=E_{i}$ for $1<i<k$. For each $i=1, \ldots, k$, let $C_{i}$ be a small major subset of $E_{i}$ as in Theorem 2.6: Define $\psi\left(e_{i}\right)=E_{i}-C_{i}$ if $e_{i}$ is not innermost, and $\psi\left(e_{i}\right)=E_{i}$ otherwise.

Subcase 3. Let $\left\{e_{i}: 1<i<k\right\}$ be ordered so that $e_{i}$ is an atom of $\left(L_{1}, A_{1}\right)$ for $1<i<r$ and $e_{i}$ is an atom of $\left(L_{2}, A_{2}\right)$ for $r<i<k$. Let $K, H_{1}$, and $H_{2}$ be as in Theorem 2.21. Let $E_{r+1}, \ldots, E_{k}$ be a Friedberg splitting of $\mathrm{H}_{2}$ as in Theorem 2.4. Define $\psi\left(L\left(e_{i}\right)\right)=E_{i}$ for $i=r+1, \ldots, k$. For each $i=r+$ $1, \ldots, k$, let $C_{i}$ be a small major subset of $E_{i}$ as in Theorem 2.6: Define $\psi\left(e_{i}\right)=E_{i}-C_{i}$ if $e_{i}$ is not innermost, and $\psi\left(e_{i}\right)=E_{i}$ otherwise. Let $E_{1}, \ldots, E_{r}$ be an Owings splitting of $H_{1}$ above $K$ as in Theorem 2.11. For $i=1, \ldots, r$, define $\psi\left(L\left(e_{i}\right)\right)=E_{i}$ : Define $\psi\left(e_{i}\right)=E_{i}-K$ if $e_{i}$ is not innermost, and $\psi\left(e_{i}\right)=E_{i}$ otherwise.

Case 3. Otherwise. Let $D=\psi\left(L\left(e^{+}\right)\right)-\psi\left(e^{+}\right)$. (Note that $D$ is r.e. by Case 1 and Case 2 of the construction.) By Theorem 2.2, let $E_{1}, \ldots, E_{k}$ be a Friedberg splitting of $D$ as in Theorem 2.4 relativized to $\psi\left(L\left(e^{+}\right)\right)$. Define $\psi\left(L\left(e_{i}\right)\right)=E_{i}$ for $i=1, \ldots, k$. For each $i=1, \ldots, k$, by Theorem 2.2, choose a small major subset $C_{i}$ of $E_{i}$ relative to $\psi\left(L\left(e^{+}\right)\right)$by Theorem 2.6 relativized to $\psi\left(L\left(e^{+}\right)\right)$: Define $\psi\left(e_{i}\right)=E_{i}-C_{i}$ if $e_{i}$ is not innermost, and $\psi\left(e_{i}\right)=E_{i}$ otherwise.

This completes the construction. We leave it to the reader to verify inductively that the construction is well defined. Let $\left(\mathscr{P}^{\prime}, \mathscr{B}^{\prime}\right)$ be the sub- $d$ lattice of $(\mathcal{E}, \mathcal{Q})$ generated by $\{\psi(L(e)): e$ as in some fixed subcase $\}$.

LeMMA 3.1. If $\left(\mathscr{P}^{\prime}, \mathscr{B}^{\prime}\right)$ is of type 1 , then $\left(\mathscr{P}^{\prime}, \mathscr{B}^{\prime}\right) \simeq(L, A)$.

Proof. See Lachlan [3, p. 138].

LEMMA 3.2. Let $\left(\mathscr{P}^{\prime}, \mathscr{B}^{\prime}\right)$ be of type 2 or type 3. For $i=1,2$, let $J_{i}=$ $\cup\left\{\psi(e)\right.$ : $e$ an atom of $\left.\left(L_{i}, A_{i}\right)\right\}$, and let $\left(\mathscr{P}_{i}, \mathscr{B}_{i}\right)=\left(\mathscr{P}^{\prime}, \mathscr{B}^{\prime}\right) \mid J_{i}$. Then $\left(\mathscr{P}_{i}, \mathscr{B}_{i}\right)$ $\simeq\left(L_{i}, A_{i}\right)$. 
Proof. Exactly as in the proof of [3, Theorem 1]. Our construction has all the properties of Lachlan's construction.

Let $\left(\mathscr{P}^{\prime}, \mathscr{B}^{\prime}\right)$ be of type 2 or type 3 , and let $\left(\mathscr{P}_{i}, \mathscr{B}_{i}\right)$ be as in Lemma 3.2 for $i=1,2$. Since the given fusion of $\left(L_{1}, A_{1}\right)$ and $\left(L_{2}, A_{2}\right)$ over $(L, A)$ is isomorphic to $(L, A) \mid a_{0}^{\prime}$, the same fusion of $\left(\mathscr{P}_{1}, \mathscr{B}_{1}\right)$ and $\left(\mathscr{P}_{2}, \mathscr{B}_{2}\right)$ over $(L, A)$ is also isomorphic to $(L, A) \mid a_{0}^{\prime}$. Denote this fusion by $\left(\mathscr{P}^{*}, \mathscr{B}^{*}\right)$. Let $(\mathscr{P}, \mathscr{B})$ be the sub- $d$-lattice of $(\mathcal{E}, \mathbb{Q})$ generated by the elements of $\mathscr{P} *$. Note that $(\mathscr{P}, \mathscr{B})$ is uniquely determined by the construction and that $(\mathscr{P}, \mathscr{B}) \mid \psi\left(a_{0}^{\prime}\right)=\left(\mathscr{P}^{*}, \mathscr{B}^{*}\right)$.

Recall that for $Q \in \mathscr{B}, \mathscr{P}(Q)$ is the smallest element of $\mathscr{P}$ containing $Q$.

LEMMA 3.3. $(\mathscr{P}, \mathscr{B}) \simeq(L, A)$.

Proof. If $(\mathscr{P}, \mathscr{B})$ is of type 1 , then $(\mathscr{P}, \mathscr{B})=\left(\mathscr{P}^{\prime}, \mathscr{B}^{\prime}\right)$ so the lemma follows from Lemma 3.1. Assume that $(\mathscr{P}, \mathscr{B})$ is of type 2 or type 3 . As $(\mathscr{P}, \mathscr{B})\left|\psi\left(a_{0}^{\prime}\right)=\left(\mathscr{P}^{*}, \mathscr{B}^{*}\right) \simeq(L, A)\right| a_{0}^{\prime}$, we need only show that $\psi\left(a_{0}\right)$ is the unique outermost atom of $(\mathscr{P}, \mathscr{B})$; i.e., that $\mathscr{P}\left(\psi\left(a_{0}\right)\right)=\omega$. Suppose that $\psi\left(a_{0}\right) \subseteq S \in \mathscr{P}$. Let $D$ be any outermost atom of $\left(\mathscr{P}^{*}, \mathscr{B}^{*}\right)$. It suffices to show that $S \cap D \neq * \varnothing$. There must be some outermost atom of either $\left(L_{1}, A_{1}\right)$ or $\left(L_{2}, A_{2}\right)$ such that $\psi(e) \subseteq D$. Fix such an $e$. Then $\psi(e)$ is defined in Case 2, Subcase 2 or Subcase 3. If $e$ is innermost, then $\psi(e)=E_{i}$ is part of a Friedberg or Owings splitting of an r.e. nonrecursive set, so is not recursive. As $\psi\left(a_{0}\right) \subseteq S, S \cap E_{i} \neq * \varnothing$ (else $E_{i}$ would be recursive), so $S \cap D \neq * \varnothing$. If $e$ is not innermost and $\psi(e)$ is defined in Subcase 2, or if $\psi(e)$ is defined in Subcase 3 and $e \in A_{2}$, then $\psi(e)=E_{i}-C_{i}$ where $C_{i}$ is a major subset of $E_{i}$. If $e$ is not innermost and $\psi(e)$ is defined in Case 2, Subcase 3 and $e \in A_{1}$, then $\psi(e)=E_{i}-K$ for some $i$. Since $K$ is a major subset of $H_{0} \supseteq E_{i}$ and $E_{i}-K$ is infinite, $E_{i} \cap K$ must be a major subset of $E_{i}$. Let $T=S \cup \psi(e)^{\prime}$. As $\psi\left(a_{0}\right) \subseteq S, T$ is r.e., and $E_{i}^{\prime} \subseteq T$. Thus we must have $\psi(e) \subseteq T$. But then $\psi(e) \subseteq S$, so $S \cap D \neq * \varnothing$.

Let $\left(L^{\prime}, A^{\prime}\right)$ be a refinement of $(L, A)$ such that $\left(L^{\prime}, A^{\prime}\right) \simeq\left(\mathscr{P}^{\prime}, \mathscr{B}^{\prime}\right)$. Let $\left(L^{\prime \prime}, A^{\prime \prime}\right)$ be a finite separated refinement of $\left(L^{\prime}, A^{\prime}\right)$ and assume that the diagram

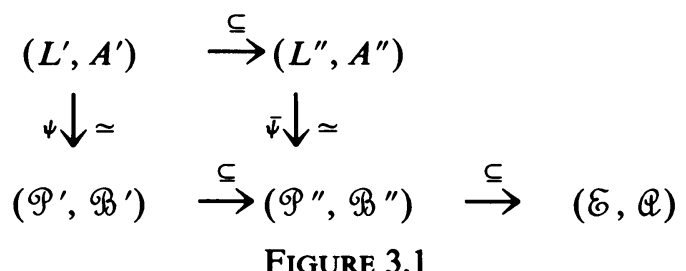

FIGURE 3.1

commutes, where $\left(\mathcal{P}^{\prime \prime}, \mathscr{B}^{\prime \prime}\right)$ has no finite atoms. We show that the following conditions are necessary (recall that $\operatorname{Max}(p)$ means that $p$ is interpreted in $(\mathcal{E}, \mathbb{Q})$ by a maximal set):

(3.3) Let $p_{2} \in A^{\prime \prime}$ be given such that $\operatorname{Max}\left(p_{2}\right)$. Then $p_{2} \in L^{\prime \prime}$ and $p_{2}^{\prime}$ is an atom of $\left(L^{\prime \prime}, A^{\prime \prime}\right)$. Let $b_{1}$ be the atom of $\left(L^{\prime}, A^{\prime}\right)$ such that $p_{2}^{\prime} \subseteq b_{1}$. Then either $b_{1}$ is an innermost atom of $\left(L^{\prime}, A^{\prime}\right)$, or $(\mathscr{P}, \mathscr{B})$ is of type 2 with 
$\left(L_{2}, A_{2}\right)=\varnothing$ and $b_{1}=a_{0}$ and $L^{\prime \prime}\left(p_{2}^{\prime}\right) \cap a_{0}^{\prime}=0$, or $(\mathcal{P}, \mathscr{B})$ is of type 1 and $p_{2}^{\prime}=b_{1}=a_{0}$. In the latter case, we must have $\operatorname{Max}\left(p_{2}\right)$.

(3.4) Let $(\mathcal{P}, \mathscr{B})$ be of type 2 . Then there is a unique outermost atom $a^{2}$ of ( $\left.L^{\prime \prime}, A^{\prime \prime}\right)$ such that $a^{2} \subseteq a_{0}$ and $L^{\prime \prime}\left(a^{2}\right) \cap a_{0}^{\prime} \neq 0$. Furthermore, if $\left(L_{2}, A_{2}\right) \neq$ $\varnothing$, then $a^{2}$ is the only outermost atom of $\left(L^{\prime \prime}, A^{\prime \prime}\right)$ which is contained in $a_{0}$.

(3.5) Let $(\mathscr{P}, \mathscr{B})$ be of type 3 . Then there is a unique outermost atom $a^{2}$ of $\left(L^{\prime \prime}, A^{\prime \prime}\right)$ such that $L^{\prime \prime}\left(a^{2}\right) \cap d \neq 0$ for some outermost atom $d$ of $\left(L_{1}, A_{1}\right)$. Furthermore, for this $a^{2}, L^{\prime \prime}\left(a^{2}\right) \cap d \neq 0$ for every outermost atom $d$ of $\left(L_{1}, A_{1}\right)$.

(3.6) Let $a_{1}, b_{1}$ be atoms of $\left(L^{\prime}, A^{\prime}\right)$ such that $a_{1} \prec_{j} b_{1}$ and $a_{1}$ is not innermost. Let $a_{2} \subseteq a_{1}$ be an atom of $\left(L^{\prime \prime}, A^{\prime \prime}\right)$. Then there is an atom $b_{2} \subseteq b_{1}$ of ( $\left.L^{\prime \prime}, A^{\prime \prime}\right)$ such that $a_{2} \prec b_{2}$.

(3.7) Let $a_{1}, b_{1}$ be atoms of $\left(L^{\prime}, A^{\prime}\right)$ such that $a_{1} \prec_{j} b_{1}$. Let $b_{2} \subseteq b_{1}$ be an atom of $\left(L^{\prime \prime}, A^{\prime \prime}\right)$. Then there is an atom $a_{2} \subseteq a_{1}$ of $\left(L^{\prime \prime}, A^{\prime \prime}\right)$ such that $a_{2} \prec b_{2}$, unless either $(\mathscr{P}, \mathscr{B})$ is of type $2, b_{1}=a_{0}, b_{2} \neq a^{2}, a_{1} \in A_{1}$, and $\left(L_{1}, A_{1}\right) \neq \varnothing$ (in which case no such $a_{2}$ can exist), or $(\mathscr{P}, \mathscr{B})$ is of type 3 , $b_{1}=a_{0}, b_{2} \neq a^{2}$ and $a_{1} \in A_{1}$.

(3.8) Let $a_{2}, b_{2}$ be atoms of $\left(L^{\prime \prime}, A^{\prime \prime}\right)$ such that $a_{2} \prec_{j} b_{2}$. Let $a_{1}, b_{1}$ be atoms of $\left(L^{\prime}, A^{\prime}\right)$ such that $a_{2} \subseteq a_{1}, b_{2} \subseteq b_{1}$. Then $a_{1}=b_{1}$ or $a_{1} \prec_{j} b_{1}$.

(3.9) Let $(\mathcal{P}, \mathscr{B})$ be of type 1 . Then if $a_{2} \subseteq a_{0}$ is an atom of $\left(L^{\prime \prime}, A^{\prime \prime}\right)$, then $a_{2}=a_{0}$.

(3.10) Let $(\mathcal{P}, \mathscr{B})$ be of type 2 . Let $b_{2}$ be an atom of $\left(L^{\prime \prime}, A^{\prime \prime}\right)$ such that $b_{2} \subseteq a_{0}$ and $b_{2} \neq a^{2}$. Then $\left(L^{\prime}, A^{\prime}\right) \cap\left(L^{\prime \prime}\left(b_{2}\right) \cap a_{0}^{\prime}\right) \simeq\left(L_{2}, A_{2}\right)$ and $\left(L^{\prime}, A^{\prime}\right)$ $\cap\left(L^{\prime \prime}\left(b_{2}\right) \cap a_{0}^{\prime}\right) \subseteq\left(L_{2}, A_{2}\right)$.

Proof of (3.3). Fix $p_{2}$ and $b_{1}$ as in (3.3). Then $\bar{\psi}\left(p_{2}\right)$ must be a maximal set, hence $p_{2} \in L$. As $\bar{\psi}\left(p_{2}^{\prime}\right)$ is cohesive, $p_{2}^{\prime}$ must be an atom of $\left(L^{\prime \prime}, A^{\prime \prime}\right)$. Suppose first that $b_{1} \neq a_{0}$. If $b_{1}$ is not innermost, then $\psi\left(b_{1}\right)=V-U$ for r.e. sets $U, V$ such that $U$ is a major subset of $V$. By Remark 2.7, $V-U$ is pure $d$-r.e. hence cannot contain the co-r.e. set $\bar{\psi}\left(p_{2}^{\prime}\right)$. Hence in this case, $b_{1}$ must be an innermost atom of $\left(L^{\prime}, A^{\prime}\right)$. Suppose next that $b_{1}=a_{0}$. If $(\mathcal{P}, \mathscr{B})$ is of type 1 , then $\psi\left(b_{1}^{\prime}\right)$ is a maximal set, so we must have $p_{2}^{\prime}=b_{1}$ and $\operatorname{Max}\left(p_{2}\right)$. If $(\mathscr{P}, \mathscr{B})$ is of type 2 with $\left(L_{2}, A_{2}\right) \neq \varnothing$ or of type 3 , then $\psi\left(b_{1}^{\prime}\right)$ is atomless, so we cannot have $\operatorname{Max}\left(p_{2}\right)$. If $(\mathscr{P}, \mathscr{B})$ is of type 2 and $\left(L_{2}, A_{2}\right)=\varnothing$, then $\psi\left(b_{1}\right)=$ $B_{1}^{\prime}=B_{2} \cup B^{\prime}$. As $B$ is atomless, we must have $\bar{\psi}\left(p_{2}^{\prime}\right) \subseteq B_{2}$. But then $\bar{\psi}\left(L^{\prime \prime}\left(p_{2}^{\prime}\right)\right) \cap B_{2}=\bar{\psi}\left(L^{\prime \prime}\left(p_{2}^{\prime}\right)\right)-B_{1}$ is r.e., so $\bar{\psi}\left(L^{\prime \prime}\left(p_{2}^{\prime}\right)\right)-B_{1} \in \mathcal{P}{ }^{\prime \prime}$. Hence $L^{\prime \prime}\left(p_{2}^{\prime}\right) \cap a_{0}^{\prime}=0$.

Proof of (3.4). If $\left(L_{2}, A_{2}\right) \neq \varnothing$, then $\psi\left(a_{0}\right)$ is $r$-cohesive, hence immune by Remark 2.10. Any outermost atom $b_{2}$ of $\left(L^{\prime \prime}, A^{\prime \prime}\right)$ must, therefore, satisfy $L^{\prime \prime}\left(b_{2}\right) \cap a_{0}^{\prime} \neq 0$. Thus it suffices to show that there is a unique outermost atom $a^{2} \subseteq a_{0}$ of $\left(L^{\prime \prime}, A^{\prime \prime}\right)$. As $\psi\left(a_{0}^{\prime}\right)$ is not recursive, there must be an atom $a^{2} \subseteq a_{0}$ of $\left(L^{\prime \prime}, A^{\prime \prime}\right)$ such that $L^{\prime \prime}\left(a^{2}\right) \cap a_{0}^{\prime} \neq 0$. Fix such an $a^{2}$, with the property that $\bar{\psi}\left(L^{\prime \prime}\left(a^{2}\right)\right) \cap B \neq \varnothing$. Let $E=\left\{e_{2} \in A^{\prime \prime}: e_{2} \subseteq a_{0} \& e_{2} \neq\right.$ $a^{2} \& e_{2}$ is an outermost atom of $\left.\left(L^{\prime \prime}, A^{\prime \prime}\right)\right\}$. If $E=\varnothing$, then we are done. Otherwise, let $S_{0}=\bar{\psi}\left(L^{\prime \prime}\left(a^{2}\right)\right)$ and $S_{1}=\bigcup\left\{\bar{\psi}\left(L^{\prime \prime}\left(e_{2}\right)\right): e_{2} \in E\right\}$. Then $S_{0}$ 
and $S_{1}$ are r.e. sets such that $\psi\left(a_{0}\right) \subseteq S_{0} \cup S_{1}$, and $\psi\left(a_{0}\right)-S_{i} \neq{ }^{*} \varnothing$ for $i=0,1$. Let $G=\psi\left(a_{0}^{\prime}\right)$. By Theorem 2.1, there is a partition $\left\{T_{0}, T_{1}\right\}$ of $\omega$ such that $T_{0} \subseteq S_{0} \cup G, T_{1} \subseteq S_{1}$, and $\psi\left(a_{0}\right)-S_{i}=\psi\left(a_{0}\right)-T_{i}$ for $i=0$, 1. If $\left(L_{2}, A_{2}\right) \neq \varnothing$, this is impossible since $G$ is $r$-maximal, hence $E=\varnothing$ in this case. If $\left(L_{2}, A_{2}\right)=\varnothing$, then $G^{\prime}=B^{\prime} \cup B_{2}$ with $B r$-maximal, so we must have $B^{\prime} \subseteq{ }^{*} T_{0}$. Hence $T_{1} \subseteq \subseteq^{*} G \cup B_{2}$. Let $e_{2} \in E$. Then $\bar{\psi}\left(L^{\prime \prime}\left(e_{2}\right)\right)-G \in \mathscr{B}^{\prime \prime}$ and $\bar{\psi}\left(L^{\prime \prime}\left(e_{2}\right)\right)-G=\bar{\psi}\left(L^{\prime \prime}\left(e_{2}\right)\right) \cap B_{2}$ is r.e. hence in $\mathcal{P} "$. Thus $L^{\prime \prime}(e) \cap a_{0}^{\prime}=$ 0 .

Proof of (3.5). Let $d$ be any outermost atom of $\left(L_{1}, A_{1}\right)$ and let $T=$ $\cup\left\{\bar{\psi}\left(L^{\prime \prime}\left(c_{2}\right)\right): c_{2} \subseteq a_{0}\right.$ is an atom of $\left.\left(L^{\prime \prime}, A^{\prime \prime}\right)\right\} . K \cup\left(T-H_{0}\right)$ cannot be r.e., since $K$ is a major subset of $H_{0}, H_{0}^{\prime} \subseteq H_{1} \cup\left(T-H_{0}\right)$ and $\left(H_{0}-K\right) \cap\left(H_{1}\right.$ $\left.\cup K \cup\left(T-H_{0}\right)\right)=\varnothing$. If $d$ is an innermost atom of $\left(L_{1}, A_{1}\right)$, then $\psi(d)$ is part of an Owings splitting of $H_{0}$ above $K$, so $K \cup(T-\psi(d))$ cannot be r.e. Thus $T-\psi(d)$ is not r.e., so there is an atom $a^{2} \subseteq a_{0}$ of $\left(L^{\prime \prime}, A^{\prime \prime}\right)$ such that $L^{\prime \prime}\left(a^{2}\right) \cap d \neq 0$. If $d$ is not innermost, then there are r.e. sets $U \subset V \subseteq H_{0}$ such that $\psi(d)=V-U, H_{0}-V$ is r.e., and $U$ is a major subset of $V$. As $V^{\prime} \subseteq\left(H_{0}-V\right) \cup H_{1} \cup T$ and $\psi(d) \cap\left(H_{1} \cup\left(H_{0}-V\right)\right)=\varnothing$, we must have $V-U=\psi(d) \subseteq \subseteq^{*} T$. Hence again there is an atom $a^{2} \subseteq a_{0}$ of $\left(L^{\prime \prime}, A^{\prime \prime}\right)$ such that $L^{\prime \prime}\left(a^{2}\right) \cap d \neq 0$. Fix such an $a^{2}$.

Suppose that for some outermost atom $c$ of $\left(L_{1}, A_{1}\right)$, there is an atom $c_{2} \subseteq a_{0}$ of $\left(L^{\prime \prime}, A^{\prime \prime}\right)$ such that $c_{2} \neq a^{2}$ and $L^{\prime \prime}\left(c_{2}\right) \cap c \neq 0$, for the sake of obtaining a contradiction. Let $S=\cup\left\{\bar{\psi}\left(L^{\prime \prime}\left(e_{2}\right)\right): e_{2} \neq a^{2}\right.$ is an outermost atom of $\left.\left(L^{\prime \prime}, A^{\prime \prime}\right)\right\}$. Note that as $H$ is hhs, $a^{2}$ must be an outermost atom of $\left(L^{\prime \prime}, A^{\prime \prime}\right)$, so $\bar{\psi}\left(L^{\prime \prime}\left(a^{2}\right)\right) \cap S=\varnothing$ as $\left(L^{\prime \prime}, A^{\prime \prime}\right)$ is separated. Hence $S$ and $\bar{\psi}\left(L^{\prime \prime}\left(a^{2}\right)\right)$ are recursive sets partitioning $\omega$. As $H_{0}-K$ is $r$-cohesive and $\psi(c) \cap\left(H_{0}-K\right) \neq * \varnothing$, we must have $H_{0}-K \subseteq \subseteq^{*}$. But as $\psi(d) \cap\left(H_{0}-\right.$ $K) \cap \bar{\psi}\left(L^{\prime \prime}\left(a^{2}\right)\right) \neq \neq^{*} \varnothing$ and $H_{0}-K$ is $r$-cohesive, we must have $H_{0}-$ $K \subseteq \subseteq^{*} \bar{\psi}\left(L^{\prime \prime}\left(a^{2}\right)\right)$. Hence $H_{0}-K$ must be finite, a contradiction.

Proof of (3.6). Fix $a_{1}, b_{1}, a_{2}$ as in (3.6). Then there are r.e. sets $U, V, X, Y$ such that $U \subset V \subseteq X \subset Y, \psi\left(a_{1}\right)=V-U, \psi\left(b_{1}\right)=Y-X, X-V$ is r.e., and $U$ is a major subset of $V$ relative to $Y$. Since $U\left\{L^{\prime \prime}\left(b_{2}\right): b_{2} \subseteq b_{1}\right.$ is an atom of $\left.\left(L^{\prime \prime}, A^{\prime \prime}\right)\right\} \cup(X-V)=T$ is an r.e. set containing $Y-V$, we must have $V-U \subseteq * T$. As $(V-U) \cap(X-V)=\varnothing$ and $\bar{\psi}\left(a_{2}\right) \subseteq V-U$, we must have $\bar{\psi}\left(a_{2}\right) \subseteq \subseteq^{*} \bar{\psi}\left(L^{\prime \prime}\left(b_{2}\right)\right)$ for some atom $b_{2} \subseteq b_{1}$ of $\left(L^{\prime \prime}, A^{\prime \prime}\right)$. For such a $b_{2}, a_{2}<b_{2}$.

Proof of (3.7). Fix $a_{1}, b_{1}, b_{2}$ as in (3.7). First consider the cases where either $b_{1} \neq a_{0}$ or $(\mathscr{P}, \mathscr{B})$ is of type 1 , or $(\mathscr{P}, \mathscr{B})$ is of type 2 with $\left(L_{1}, A_{1}\right)=$ $\varnothing$. Then there are r.e. sets $U, V, X, Y$ such that $U \subset V \subseteq X \subset Y, \psi\left(a_{1}\right)=V$ $-U, \psi\left(b_{1}\right)=Y-X, X-V$ is r.e., and if $U \neq \varnothing$ then $U$ is a small subset of $V$ relative to $Y . \bar{\psi}\left(L^{\prime \prime}\left(b_{2}\right)\right) \cap(Y-X)=\left(\bar{\psi}\left(L^{\prime \prime}\left(b_{2}\right)\right) \cap Y\right)-X$ is infinite but not r.e. since $Y-X$ is seen to be immune. As $V$ was obtained through a Friedberg splitting of $X$ relative to $Y,\left(\bar{\psi}\left(L^{\prime \prime}\left(b_{2}\right)\right) \cap Y\right)-V$ cannot be r.e. Hence $\bar{\psi}\left(L^{\prime \prime}\left(b_{2}\right)\right) \cap V$ is infinite. If $U=\varnothing$, then we are done. Otherwise, 
since $U$ is a small subset of $V$ relative to $Y$, if $\bar{\psi}\left(L^{\prime \prime}\left(b_{2}\right)\right) \cap(V-U)={ }^{*} \varnothing$ then $\bar{\psi}\left(L^{\prime \prime}\left(b_{2}\right)\right)-V=\left(\bar{\psi}\left(L^{\prime \prime}\left(b_{2}\right)\right) \cap Y\right)-V$ would have to be r.e., but we have shown this not to be the case. Hence $\bar{\psi}\left(L^{\prime \prime}\left(b_{2}\right)\right) \cap(V-U) \neq \neq^{*} \varnothing$. Thus there is an atom $a_{2}$ of $\left(L^{\prime \prime}, A^{\prime \prime}\right)$ such that $a_{2} \prec b_{2}$.

Next consider the case where $b_{1}=a_{0},(\mathscr{P}, \mathscr{B})$ is of type 2 , and $\left(L_{1}, A_{1}\right) \neq$ $\varnothing$. If $b_{2} \neq a^{2}$ and $a_{1} \in A_{1}$, then $\psi\left(a_{1}\right) \subseteq B_{1}$ and by Theorem 2.15, $B_{2} \cup$ $\left(\bar{\psi}\left(L^{\prime \prime}\left(b_{2}\right)\right)-\psi\left(a_{0}^{\prime}\right)\right)$ is r.e. Hence $L^{\prime \prime}\left(b_{2}\right) \cap a_{1}=0$, else

$$
\begin{aligned}
\bar{\psi}\left(L^{\prime \prime}\left(b_{2}\right)\right)-\psi\left(a_{0}^{\prime}\right)= & {\left[B_{2} \cup\left(\bar{\psi}\left(L^{\prime \prime}\left(b_{2}\right)\right)-\psi\left(a_{0}^{\prime}\right)\right)\right] } \\
& \cap\left[B_{1} \cup\left(\bar{\psi}\left(L^{\prime \prime}\left(b_{2}\right)\right)-\psi\left(a_{0}\right)\right)\right]
\end{aligned}
$$

would be an infinite r.e. subset of the immune set $B^{\prime}$, which is impossible. Suppose that $a_{1} \in A_{2}$. Then there are r.e. sets $U, V, X, Y$ such that $U \subset V \subseteq$ $X \subset Y, \psi\left(a_{1}\right)=V-U, \psi\left(b_{1}\right)=Y-X, X-V$ is r.e., and if $U \neq \varnothing$ then $U$ is a small subset of $V$ relative to $Y$.

$$
\bar{\psi}\left(L^{\prime \prime}\left(b_{2}\right)\right) \cap(Y-X)=\left(\bar{\psi}\left(L^{\prime \prime}\left(b^{2}\right)\right) \cap Y\right)-X
$$

is infinite, and is not r.e. as $Y-X$ is immune since $\left(L_{2}, A_{2}\right) \neq \varnothing$. By Theorem $2.15, \bar{\psi}\left(L^{\prime \prime}\left(b_{2}\right)\right)-B_{2}$ is not r.e., else $\bar{\psi}\left(L^{\prime \prime}\left(b_{2}\right)\right)-B$ would be an infinite r.e. subset of the immune set $B^{\prime}$. As $V$ is obtained from a Friedberg splitting of $B_{2}, \bar{\psi}\left(L^{\prime \prime}\left(b_{2}\right)\right)-V$ cannot be r.e. We can now proceed as in the preceding paragraph. Finally, assume that $a_{1} \in A_{1}$ and $b_{2}=a^{2}$. By (3.4), $\bar{\psi}\left(L^{\prime \prime}\left(b_{2}\right)\right) \cap \psi\left(a_{0}^{\prime}\right) \neq * \varnothing$. If $\left(L_{2}, A_{2}\right)=\varnothing$, then $\psi\left(a_{0}^{\prime}\right)=B_{1}$ so $\bar{\psi}\left(L^{\prime \prime}\left(b_{2}\right)\right) \cap$ $B_{1} \neq * \varnothing$. And if $\left(L_{2}, A_{2}\right) \neq \varnothing$, then by (3.4), $a_{0} \subseteq L^{\prime \prime}\left(b_{2}\right)$; we note that from Theorem $2.15, B_{1}$ is not recursive, so again we must have $\bar{\psi}\left(L^{\prime \prime}\left(b_{2}\right)\right) \cap$ $B_{1} \neq^{*} \varnothing$, else $B_{1}^{\prime}=B_{2} \cup \bar{\psi}\left(L^{\prime \prime}\left(b_{2}\right)\right)$ would be r.e. Hence $\bar{\psi}\left(L^{\prime \prime}\left(b_{2}\right)\right)-B_{1}$ cannot be r.e. There are r.e. sets $U, V, X, Y$ such that $U \subset V \subseteq X \subset Y$, $\psi\left(a_{1}\right)=V-U, \psi\left(b_{1}\right)=Y-X, X-V$ is r.e., and if $U \neq \varnothing$ then $U$ is a small subset of $V$ relative to $Y$. As $V$ is obtained from a Friedberg splitting of $B_{1}, \bar{\psi}\left(L^{\prime \prime}\left(b_{2}\right)\right)-V$ cannot be r.e. We can now proceed as in the preceding paragraph.

Finally suppose that $(\mathscr{P}, \mathscr{B})$ is of type 3 and that $b_{1}=a_{0}$. As $\psi\left(a_{0}^{\prime}\right)$ is hhs, we must have $b_{2}^{\prime} \in L^{\prime \prime}$. Suppose first that $a_{1} \in A_{2}$. Then there are r.e. sets $U$, $V, X, Y$ such that $U \subset V \subseteq X \subset Y, \psi\left(a_{1}\right)=V-U, \psi\left(b_{1}\right)=Y-X, X-$ $V$ is r.e., and if $U \neq \varnothing$ then $U$ is a small subset of $V$ relative to $Y$. As $\psi\left(a_{0}\right)=H^{\prime}$ and $H$ is hhs, $H^{\prime}$ is not immune by Remark 2.10. Hence $\bar{\psi}\left(L^{\prime \prime}\left(b_{2}\right)\right) \cap H \neq \neq^{*} \varnothing$ so $\bar{\psi}\left(L^{\prime \prime}\left(b_{2}\right)\right)-H$ is not r.e. By Theorem 2.21, $\bar{\psi}\left(L^{\prime \prime}\left(b_{2}\right)\right)-H_{2}$ cannot be r.e. And as $V$ is part of a Friedberg splitting of $H_{2}$, $\bar{\psi}\left(L^{\prime \prime}\left(b_{2}\right)\right)-V$ is not r.e. We now proceed as in the first paragraph of this proof. Suppose that $a_{1} \in A_{1}$ and $L^{\prime \prime}\left(b_{2}\right) \cap d \neq 0$ for some outermost atom $d$ of $\left(L_{1}, A_{1}\right)$. As $a_{1}$ is an outermost atom of $\left(L_{1}, A_{1}\right)$, by (3.5), $b_{2}=a^{2}$ and $L^{\prime \prime}\left(b_{2}\right) \cap a_{1} \neq 0$. Hence there is an atom $a_{2} \subseteq a_{1}$ such that $a_{2} \prec b_{2}$. 
Proof of (3.8). Let $a_{1}, b_{1}, a_{2}, b_{2}$ be as in (3.8). Assume that $a_{1} \neq b_{1}$ and $a_{1}$ is not just within $b_{1}$ for the sake of obtaining a contradiction. Note that if $K$ is a small subset of $H_{0}$, and $E_{1}, \ldots, E_{n}$ are r.e. sets partitioning $H_{0}$ and splitting $H_{0}-K$, then for $i=1, \ldots, n, K \cap E_{i}$ is a small subset of $H_{0} \cap E_{i}$. Thus there is an atom $c_{1}$ of $\left(L^{\prime}, A^{\prime}\right)$ such that $a_{1} \prec c_{1}$ and $c_{1} \prec_{j} b_{1}$. Furthermore, there are r.e. sets $U, V, X, Y$ such that $U \subset V \subseteq X \subset Y, \psi\left(c_{1}\right)=V-U$, $\psi\left(b_{1}\right)=Y-X, X-V$ is r.e., and $U$ is a small subset of $V$ relative to $Y$. Let $T=\left\{c_{2}: c_{2} \subseteq L^{\prime \prime}\left(b_{2}\right) \cap c_{1}\right.$ and $c_{2}$ is an atom of $\left.\left(L^{\prime \prime}, A^{\prime \prime}\right)\right\}$ and $S=$ $\cup\left\{\bar{\psi}\left(L^{\prime \prime}\left(c_{2}\right)\right): c_{2} \in T\right\}$. Then $S \in \mathcal{P}$ " and $\bar{\psi}\left(L^{\prime \prime}\left(b_{2}\right)\right) \cap \psi\left(c_{1}\right) \subseteq S$, so we must have

$$
S \cup\left(\bar{\psi}\left(L^{\prime \prime}\left(b_{2}\right)\right)-\psi\left(L^{\prime}\left(c_{1}\right)\right)\right)=W \in \mathcal{P}^{\prime \prime}
$$

by smallness. Hence $\bar{\psi}\left(L^{\prime \prime}\left(b_{2}\right)\right) \subseteq W$, so

$$
\bar{\psi}\left(L^{\prime \prime}\left(b_{2}\right)\right) \cap \psi\left(L^{\prime}\left(c_{1}\right)\right) \subseteq S .
$$

As $a_{2} \subseteq L^{\prime \prime}\left(b_{2}\right) \cap L^{\prime}\left(c_{1}\right), \psi\left(a_{2}\right) \subseteq \bar{\psi}\left(L^{\prime \prime}\left(c_{2}\right)\right)$ for some atom $c_{2} \in T$ of $\left(L^{\prime \prime}, A^{\prime \prime}\right)$. Hence there is an atom $c_{2} \subseteq c_{1}$ of $\left(L^{\prime \prime}, A^{\prime \prime}\right)$ such that $a_{2} \prec c_{2} \prec b_{2}$, yielding a contradiction.

Proof of (3.9). Clear since $\psi\left(a_{0}\right)$ is cohesive.

Proof of (3.10). Let $b_{2}$ be an atom of ( $\left.L^{\prime \prime}, A^{\prime \prime}\right)$ such that $b_{2} \subseteq a_{0}$ and $b_{2} \neq a^{2}$. Then $\bar{\psi}\left(L^{\prime \prime}\left(b_{2}\right)\right) \cap \psi\left(a_{0}\right)=S$ is infinite, hence by Theorem 2.15, $B_{2} \cup S$ is r.e. If $\left(L_{2}, A_{2}\right)=\varnothing$, then $B_{2} \subseteq \psi\left(a_{0}\right)$, hence $\bar{\psi}\left(L^{\prime \prime}\left(b_{2}\right)\right) \subseteq \psi\left(a_{0}\right)$ and (3.10) follows immediately. Suppose that $\left(L_{2}, A_{2}\right) \neq \varnothing$. Since $B_{2} \in \mathscr{P}_{2}$, we must have

$$
\left(L^{\prime}, A^{\prime}\right) \cap\left(L^{\prime \prime}\left(b_{2}\right) \cap a_{0}^{\prime}\right) \subseteq\left(L_{2}, A_{2}\right) .
$$

To complete the proof of (3.10), it suffices to show that if $D=\bar{\psi}\left(L^{\prime \prime}\left(b_{2}\right)\right)$ then $(\Re, \mathcal{C})=\left(\mathscr{P}_{2}, \mathscr{B}_{2}\right) \cap D \simeq\left(\mathscr{P}_{2}, \mathscr{B}_{2}\right)$.

Assume that $(\mathscr{R}, \mathcal{C}) \simeq\left(\mathscr{P}_{2}, \mathscr{B}_{2}\right)$ in order to obtain a contradiction. Since $(\Re, \mathcal{C}) \hookrightarrow\left(\mathscr{P}_{2}, \mathscr{B}_{2}\right)$, there must either be a canonical generator $U \in \mathscr{P}_{2}$ such that $D \cap \mathscr{B}_{2}(U)=\varnothing$, or there must be canonical generators $U, V \in \mathscr{P}_{2}$ such that $U \prec V$ but $D \cap U \approx \Re(D \cap V)$. By (3.7), the latter case can only happen if $D \cap \mathscr{B}_{2}(V)=\varnothing$. Hence we can fix a canonical generator $U$ such that $D \cap \mathscr{B}_{2}(U)=\varnothing$ and such that for no canonical generator $V \supseteq U$ of $\mathscr{P}_{2}$ is $D \cap \mathscr{B}_{2}(V)=\varnothing$. By (3.6), $\mathscr{B}_{2}(U)$ must either be an innermost or outermost atom of $\left(\mathscr{P}_{2}, \mathscr{B}_{2}\right)$.

Suppose that $\mathscr{B}_{2}(U)$ is an innermost atom of $\left(\mathscr{P}_{2}, \mathscr{B}_{2}\right)$. Then there is an r.e. set $W$ such that $U=B_{2}(U)$ is part of a Friedberg splitting of $W$ during the construction, and $W-U$ is r.e. Hence by Theorem 2.4 , as $D-U$ is r.e., $D-W$ must also be r.e. If $W$ is a canonical generator of $\left(\mathscr{P}_{2}, \mathscr{P}_{2}\right)$ and $\mathscr{B}_{2}(W)$ is an outermost atom of $\left(\mathscr{P}_{2}, \mathscr{B}_{2}\right)$, then we must have $D=\varnothing$, and so $S$ is an infinite r.e. subset of the immune set $B^{\prime}$, a contradiction. Otherwise, there is a canonical generator $X$ of $\left(\mathscr{P}_{2}, \mathscr{P}_{2}\right)$ such that $\mathscr{B}_{2}(X)=X-W$ and $\mathscr{B}_{2}(U)$ is just within $\mathscr{B}_{2}(X)$. By (3.7) $X \cap(D-W)=\varnothing$, contradicting the choice of $U$. Hence $\mathscr{B}_{2}(U)$ must be an outermost atom of $\left(\mathscr{P}_{2}, \mathscr{P}_{2}\right)$ which is 
not innermost, and so $\mathscr{B}_{2}(U)=Z-Y$ in the construction, with $Y, Z$ r.e. and $Y$ a small subset of $Z$. Since $D \cap(Z-Y)=\varnothing, D-Z$ must be r.e. By Theorem 2.4, $D-B_{2}$ must be r.e. As $D \cap B_{1}=\varnothing, D-B$ must be r.e. But then $D-B$ is an infinite r.e. subset of the immune set $B^{\prime}$, a contradiction.

4. Refinement theorems and the decision procedure. Fix a finite separated $d$-lattice $(L, A)$ with one component, a finite separated refinement $\left(L^{\prime}, A^{\prime}\right)$ of $(L, A)$, and a realization $(\mathscr{P}, \mathscr{B})$ of $(L, A)$. We will give sufficient conditions for the existence of a refinement $\left(\mathscr{P}^{\prime}, \mathscr{B}^{\prime}\right)$ of $(\mathscr{P}, \mathscr{B})$ with no finite atoms, such that the diagram

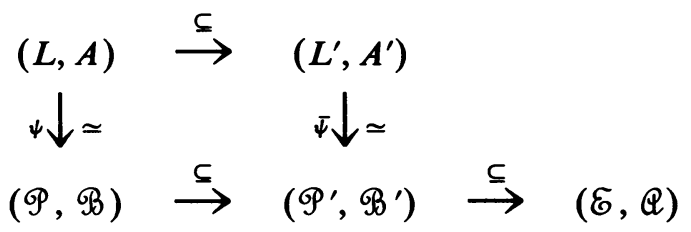

FIGURE 4.1

commutes, with appropriate elements of $\mathcal{P}^{\prime}$ maximal.

One of our principal tools will be a slight generalization of Lachlan's refinement theorem [3, Theorem 4].

Theorem 4.1 (Refinement Theorem). Let $(L, A),\left(L^{\prime}, A^{\prime}\right)$, and $(\mathscr{P}, \mathscr{B})$ be as above with $(\mathscr{P}, \mathscr{B})$ irreducible. Suppose that:

(4.1) Every outermost atom of $(L, A)$ which is not innermost is an atom of $\left(L^{\prime}, A^{\prime}\right)$.

(4.2) If $a, b$ are atoms of $(L, A)$ such that $a \prec_{j} b$ and $a$ is not innermost, and $a_{1} \subseteq a$ is an atom of $\left(L^{\prime}, A^{\prime}\right)$ then there is an atom $b_{1} \subseteq b$ of $\left(L^{\prime}, A^{\prime}\right)$ such that $a_{1}<b_{1}$.

(4.3) If $a, b$ are atoms of $(L, A)$ such that $a \prec_{j} b$, and $b_{1} \subseteq b$ is an atom of $\left(L^{\prime}, A^{\prime}\right)$, then there is an atom $a_{1} \subseteq a$ of $\left(L^{\prime}, A^{\prime}\right)$ such that $a_{1} \prec b_{1}$.

(4.4) If $a_{1}, b_{1}$ are atoms of $\left(L^{\prime}, A^{\prime}\right)$ contained in the respective atoms $a, b$ of $(L, A)$ and $a_{1} \prec_{j} b_{1}$ then $a=b$ or $a \prec_{j} b$.

Then there is a realization $\left(\mathscr{P}^{\prime}, \mathscr{B}^{\prime}\right)$ of $\left(L^{\prime}, A^{\prime}\right)$ such that the diagram of Figure 4.1 commutes. Furthermore, if $a_{1}$ is any outermost atom of $\left(\mathcal{P}^{\prime}, \mathscr{B}^{\prime}\right)$ not contained in the outermost atom of $(\mathscr{P}, \mathscr{B})$, then we can make $\bar{\psi}\left(a_{1}^{\prime}\right)$ maximal or $\bar{\psi}\left(a_{1}^{\prime}\right)$ not maximal as we desire.

Proof. Lachlan [3, Theorem 4] proves the theorem without the maximality conditions. The latter follows easily from his proof, as if $a_{1}$ is any outermost atom of $\left(\mathcal{P}^{\prime}, \mathfrak{B}^{\prime}\right)$ not contained in an outermost atom of $(\mathscr{P}, \mathscr{B})$, then by (4.2), $a_{1}$ must be contained in an innermost atom $a$ of $(\mathscr{P}, \mathscr{B})$. In this case, Lachlan chooses a recursive subset $R$ of $\psi(a)$, and defines $\bar{\psi}\left(L^{\prime}\left(a_{1}\right)\right)=R$. He then builds an isomorphic copy of $\left(L^{\prime}, A^{\prime}\right) \mid L^{\prime}\left(a_{1}\right)$ inside of $R$. If we build such a copy with a type 1 canonical realization, then $\bar{\psi}\left(a_{1}^{\prime}\right)$ will be maximal. If we build such a copy with a type 2 canonical realization, then $\bar{\psi}\left(a_{1}^{\prime}\right)$ will not be maximal. 
Fix the r.e. set $C$ such that $C^{\prime}$ is the outermost atom of $(\mathscr{P}, \mathscr{B})$. We say that $(\mathcal{P}, \mathscr{B})$ is of type 1 if $C$ is maximal. We say that $(\mathscr{P}, \mathscr{B})$ is of type 2 if $C$ is not hhs. We say that $(\mathscr{P}, \mathscr{B})$ is of type 3 if $C$ is not quasi-r-maximal. The following theorem is only a slight restatement of Theorem 4.1.

Theorem 4.2 (Type 1 Refinement Theorem). Suppose that $(\mathcal{P}, \mathscr{B})$ is irreducible of type 1, and that (3.3), (3.6), (3.7), (3.8), and (3.9) hold. Let $S=\left\{a_{1} \in A^{\prime}: a_{1}\right.$ is an outermost atom of $\left(L^{\prime}, A^{\prime}\right)$ contained in an innermost atom of $(L, A)\}$, and $T \subseteq S$. Then there exists a realization $\left(\mathscr{P}^{\prime}, \mathscr{B}^{\prime}\right)$ of ( $\left.L^{\prime}, A^{\prime}\right)$ such that Figure 4.1 commutes, and such that for all $b_{1} \in A^{\prime}, \bar{\psi}\left(b_{1}\right)$ is maximal if and only if $b_{1}^{\prime} \in T$ or $b_{1}^{\prime}$ is the outermost atom of $(L, A)$.

Proof. (3.9), (3.6), (3.7), and (3.8) immediately yield (4.1), (4.2), (4.3), and (4.4). By Theorem 4.1, we obtain $\left(\mathcal{P}^{\prime}, \mathscr{B}^{\prime}\right)$ as specified such that for all $b_{1} \in A^{\prime}, \bar{\psi}\left(b_{1}\right)$ is maximal if and only if $b_{1}^{\prime} \in T$ unless $b_{1}^{\prime}$ is the outermost atom $a_{0}$ of $(L, A)$. But in this case, by (3.3) we have $\operatorname{Max}\left(a_{0}^{\prime}\right)$ and as $C$ is maximal, by (3.9) we have $\operatorname{Max}(C)$.

Let $(\mathscr{P}, \mathscr{B})$ be irreducible of type 2 with minimizer $\left(\mathscr{P}_{2}, \mathscr{B}_{2}\right)$ and associated cominimizer $\left(\mathscr{P}_{1}, \mathscr{B}_{1}\right)$. Let $R$ be any recursive infinite coinfinite set. Note that the notions of minimizer and cominimizer straightforwardly extend to $(\mathscr{P}, \mathscr{B}) \cap R$. We say that $(\mathscr{P}, \mathscr{B}) \cap R$ has smaller degree than $(\mathscr{P}, \mathscr{B})$ as witnessed by $\left(\mathscr{P}_{1}, \mathscr{B}_{1}\right)$ and $\left(\mathscr{P}_{2}, \mathscr{B}_{2}\right)$ if one of the following holds:

(4.5) $(\mathscr{P}, \mathscr{B}) \cap R$ is reducible or $(\mathscr{P}, \mathscr{B}) \cap R$ has smaller characteristic than $(\mathcal{P}, \mathscr{B})$;

(4.6) either $\left(\mathscr{P}_{2}, \mathscr{P}_{2}\right) \cap R \not\left(\mathscr{P}_{2}, \mathscr{P}_{2}\right)$ or $\left(\mathscr{P}_{1}, \mathscr{B}_{1}\right) \cap R \not\left(\mathscr{P}_{1}, \mathscr{B}_{1}\right)$ or $\left(\mathscr{P}_{2}, \mathscr{B}_{2}\right) \cap R$ is not a minimizer of $(\mathscr{P}, \mathscr{B}) \cap R$.

As the relation "has smaller degree than" is transitive and we can effectively finitely list all possible isomorphic types of $d$-lattices embeddable into $(\mathscr{P}, \mathscr{B})$, we can effectively finitely list all possible isomorphism types of $d$-lattices of smaller degree than $(\mathscr{P}, \mathscr{B})$ which are embeddable into $(\mathscr{P}, \mathscr{B})$.

We say that $(\mathscr{P}, \mathscr{B})$ is strongly irreducible if there is a minimizer $\left(\mathscr{P}_{2}, \mathscr{B}_{2}\right)$ of $(\mathscr{P}, \mathscr{B})$ with associated cominimizer $\left(\mathscr{P}_{1}, \mathscr{B}_{1}\right)$ such that for any recursive set $R$, either

(4.7) $(\mathscr{P}, \mathscr{B}) \cap R$ is irreducible and $(\mathscr{P}, \mathscr{B}) \cap R \simeq(\mathscr{P}, \mathscr{B})$; or

(4.8) it is not the case that $(\mathscr{P}, \mathscr{B}) \cap R^{\prime}$ has smaller degree than $(\mathscr{P}, \mathscr{B})$ as witnessed by $\left(\mathscr{P}_{1}, \mathscr{B}_{1}\right)$ and $\left(\mathscr{P}_{2}, \mathscr{P}_{2}\right)$.

If $(\mathscr{P}, \mathscr{B})$ is strongly irreducible with $\left(\mathscr{P}_{1}, \mathscr{B}_{1}\right)$ and $\left(\mathscr{P}_{2}, \mathscr{B}_{2}\right)$ as above, we call $\left(\mathscr{P}_{1}, \mathscr{B}_{1}\right)$ and $\left(\mathscr{P}_{2}, \mathscr{B}_{2}\right)$ witnesses to the strong irreducibility of $(\mathscr{P}, \mathscr{B})$.

We say that $\left(\mathcal{P}^{\#}, \mathscr{B}^{\#}\right)$ is a preliminary refinement of $(\mathscr{P}, \mathscr{B})$ of type 2 if there are r.e. sets $W, D, E$ such that $W$ is not maximal $\left(\mathscr{P}_{2}, \mathscr{B}_{2}\right)=(\mathscr{P}, \mathscr{B}) \cap$ $D$ is a minimizer of $(\mathscr{P}, \mathscr{B})$ with associated cominimizer $\left(\mathscr{P}_{1}, \mathscr{B}_{1}\right)=(\mathscr{P}, \mathscr{B})$ $\cap E, C \subset^{*} W \subset^{*} \omega, D \cup(W-C)$ is r.e., $C \cup W^{\prime}$ is not r.e., and $\left(\mathscr{P}^{\#}, \mathscr{B}^{\#}\right)$ is generated by $\{X: X=W$ or $X=P \cap D$ or $X=P \cap E$ for some $P \in \mathscr{P}\}$ and has no finite atoms. 
Theorem 4.3 (Type 2 Preliminary Refinement Theorem). If $(\mathscr{P}, \mathscr{B})$ is strongly irreducible of type $2,\left(\mathscr{P}_{1}, \mathscr{B}_{1}\right)$ and $\left(\mathscr{P}_{2}, \mathscr{P}_{2}\right)$ witness the strong irreducibility of $(\mathscr{P}, \mathscr{B})$, and $\left(\mathscr{P}^{*}, \mathscr{B}^{*}\right)$ is the minimizer refinement of $(\mathscr{P}, \mathscr{B})$ for $\left(\mathcal{P}_{2}, \mathscr{B}_{2}\right)$, then $(\mathscr{P}, \mathscr{B})$ has an irreducible preliminary refinement $\left(\mathscr{P}^{\#}, \mathscr{B}^{\#}\right)$ of type 2 with $\left(\mathscr{P}^{*}, \mathscr{B}^{*}\right) \subseteq\left(\mathscr{P}^{\#}, \mathscr{B}^{\#}\right)$.

Proof. Let $(\mathscr{P}, \mathscr{B})$ be strongly irreducible of type 2 with minimizer $\left(\mathscr{P}_{2}, \mathscr{B}_{2}\right)$ and cominimizer $\left(\mathscr{P}_{1}, \mathscr{P}_{1}\right)$ witnessing the strong irreducibility of $(\mathscr{P}, \mathscr{B})$. Fix r.e. sets $D$ and $E$ such that $\left(\mathscr{P}_{2}, \mathscr{B}_{2}\right)=(\mathscr{P}, \mathscr{B}) \cap D$ and $\left(\mathscr{P}_{1}, \mathscr{B}_{1}\right)=(\mathscr{P}, \mathscr{B}) \cap E$.

Let $W$ be any r.e. set such that $W$ satisfies (3.1). If $W$ is maximal, then as $C$ is not hhs, by Theorem $2.8, C$ cannot be hhs relative to $W$. Hence there is an r.e. set $W^{*}$ such that $C \subset^{*} W^{*} \subset^{*} \omega$ and $C \cup\left(W-W^{*}\right)$ is not r.e. Hence $C \cup W^{* \prime}$ cannot be r.e. As $D \cup(W-C)$ is r.e., $D \cup\left(W^{*}-C\right)=(D \cup$ $(W-C)) \cap W^{*}$ is r.e., so $W^{*}$ satisfies (3.1) and $W^{*}$ is not maximal. Hence without loss of generality, we may assume that $W$ is not maximal. Thus there is a finite modification $\left(\mathscr{P}^{\#}, \mathscr{B}^{\sharp}\right)$ of the $d$-lattice $\left(\mathscr{P}^{+}, \mathscr{B}^{+}\right)$generated by $\{X$ : $X=W$ or $X=P \cap D$ or $X=P \cap E$ for some $P \in \mathscr{P}\}$ which is a preliminary refinement of $(\mathscr{P}, \mathscr{B})$ of type 2 . We assume that $\left(\mathscr{P}^{+}, \mathscr{B}^{+}\right)=$ $\left(\mathscr{P}^{\#}, \mathscr{B}^{\#}\right)$ without loss of generality. We must now show that $\left(\mathscr{P}^{\#}, \mathscr{B}^{\#}\right)$ is irreducible.

We now proceed by cases. First consider the case where $\left(\mathscr{P}_{1}, \mathscr{B}_{1}\right)=\varnothing$. Let $R$ be a recursive set such that $W^{\prime} \subseteq R$, and let $Q=P \cap R$ for some $P \in \mathscr{P}^{\sharp}$. Suppose that $W^{\prime}=W^{\prime} \cap R \subseteq Q$. If $(W-C) \cap R \nsubseteq Q$, then $(W-C) \cap R=\varnothing$, and so $\left(C \cap R^{\prime}\right) \cup(C \cap R) \cup W^{\prime}=C \cup W^{\prime}$ is r.e., a contradiction. Hence $(W-C) \cap R \subseteq Q$. If $C \cap R \nsubseteq Q$, then for some atom $B \subseteq C \cap R$ of $\left(\mathscr{P}^{\#}, \mathscr{B}^{\sharp}\right) \cap R, B \cap Q=\varnothing$; furthermore, we can separate $C$ and $Q$ by Theorem 2.1 , obtaining an r.e. set $S \subseteq Q$ such that $(C \cap R)-S$ is r.e. But this contradicts (3.2) since $\left(\mathscr{P}_{1}, \mathscr{B}_{1}\right)$ is a minimizer of $(\mathscr{P}, \mathscr{B})$. Hence every atom $B \subseteq C \cap R$ of $\left(\mathscr{P}^{\#}, \mathscr{B}^{\sharp}\right) \cap R$ is within $(W-C)$ $\cap R$. Choosing $S=R \cap C$ in (3.2), we see that $\left(\mathscr{P}_{1}, \mathscr{B}_{1}\right) \cap R \simeq\left(\mathscr{P}_{1}, \mathscr{B}_{1}\right)$. Hence $\left(\mathscr{P}^{\sharp}, \mathscr{B}^{\sharp}\right) \cap R \simeq\left(\mathscr{P}^{\sharp}, \mathscr{B}^{\#}\right)$, so $\left(\mathscr{P}^{\#}, \mathscr{B}^{\sharp}\right)$ is irreducible.

Finally, consider the case where $\left(\mathscr{P}_{2}, \mathscr{B}_{2}\right) \neq(\mathscr{P}, \mathscr{B}) \cap C$. Let $R$ be any recursive set such that $W^{\prime} \subseteq R^{\prime}$. If $(\mathscr{P}, \mathscr{B}) \cap R \simeq(\mathscr{P}, \mathscr{B})$, then $\left(\mathscr{P}_{1}, \mathscr{B}_{1}\right) \cap$ $R \hookrightarrow\left(\mathscr{P}_{1}, \mathscr{B}_{1}\right)$ must have smaller characteristic than $(\mathscr{P}, \mathscr{B})$ as it contains no atom which has nonempty intersection with $C^{\prime}$; and $\left(\mathscr{P}_{2}^{\prime}, \mathscr{B}_{2}^{\prime}\right)$, the sub- $d$-lattice of $(\mathcal{E}, \mathbb{Q}) \cap\left((D \cap R) \cup\left(C^{\prime} \cap R\right)\right)$ generated by $\left\{X: X=C^{\prime} \cap R\right.$ or $X=P \cap R$ for some $\left.P \in \mathscr{P}_{2}\right\}$ has smaller characteristic than $(\mathscr{P}, \mathscr{B})$ as $\left(\mathscr{P} *, \mathscr{B}^{*}\right) \cap C \hookrightarrow\left(\mathscr{P}_{2}, \mathscr{B}_{2}\right) \hookrightarrow(\mathscr{P}, \mathscr{B}) \cap C$ and $\left(\mathscr{P}_{2}, \mathscr{B}_{2}\right) \not(\mathscr{P}, \mathscr{B}) \cap C$. We note that $\left(\mathscr{P}_{2}^{\prime}, \mathscr{B}_{2}^{\prime}\right)$ is a component of $(\mathscr{P}, \mathscr{B}) \cap R$ since $[D \cup(W-C)]$ $\cap R$ is r.e. Hence if $(\mathscr{P}, \mathscr{B}) \cap R$ has the same characteristic as $(\mathscr{P}, \mathscr{B})$, $\left(\mathscr{P}_{1}, \mathscr{B}_{1}\right) \cap R$ and $\left(\mathscr{P}_{2}^{\prime}, \mathscr{B}_{2}^{\prime}\right)$ decompose it to show that $(\mathscr{P}, \mathscr{B}) \cap R$ is not irreducible, and so (4.7) fails. As $(\mathscr{P}, \mathscr{B})$ is strongly irreducible, by (4.8), $(\mathscr{P}, \mathscr{B}) \cap R^{\prime}$ cannot have smaller degree than $(\mathscr{P}, \mathscr{B})$ as witnessed by 
$\left(\mathscr{P}_{1}, \mathscr{B}_{1}\right)$ and $\left(\mathscr{P}_{2}, \mathscr{B}_{2}\right)$. Hence (4.5) and (4.6) must fail, so $(\mathscr{P}, \mathscr{B}) \cap R^{\prime}$ is irreducible, $(\mathscr{P}, \mathscr{B}) \cap R^{\prime} \simeq(\mathscr{P}, \mathscr{B}),\left(\mathscr{P}_{1}, \mathscr{B}_{1}\right) \cap R^{\prime} \simeq\left(\mathscr{P}_{1}, \mathscr{B}_{1}\right),\left(\mathscr{P}_{2}, \mathscr{B}_{2}\right) \cap$ $R^{\prime} \simeq\left(\mathscr{P}_{2}, \mathscr{B}_{2}\right)$, and $\left(\mathscr{P}_{2}, \mathscr{B}_{2}\right) \cap R^{\prime}$ is a minimizer of $(\mathscr{P}, \mathscr{B}) \cap R^{\prime}$. If $Q=P$ $\cap R^{\prime}$ for some $P \in \mathscr{P}^{\#}$ and $(W-C) \cap R^{\prime} \subseteq Q$, then as $\left(\mathscr{P}_{2}, \mathscr{P}_{2}\right) \cap R^{\prime}$ is a minimizer of $(\mathscr{P}, \mathscr{B}) \cap R^{\prime}$, we must have $D \cap R^{\prime} \subseteq Q$. Now to show that $\left(\mathscr{P}^{\sharp}, \mathscr{B}^{\sharp}\right) \cap R^{\prime} \simeq\left(\mathscr{P}^{\sharp}, \mathscr{P}^{\sharp}\right)$ and so conclude that $\left(\mathscr{P}^{\sharp}, \mathscr{B}^{\sharp}\right)$ is irreducible, it remains only to show that every atom of $\left(\mathscr{P}^{\#}, \mathscr{B}^{\sharp}\right) \cap R^{\prime}$ is within $W^{\prime}$. Let $Q=P \cap R^{\prime}$ for some $P \in \mathscr{P}^{\sharp}$ and assume that $W^{\prime} \subseteq Q$. Then since $C \cup$ $W^{\prime}$ is not r.e., $\left(C \cup W^{\prime}\right) \cap R^{\prime}$ cannot be r.e. as $C \cup W^{\prime}=C \cup\left(\left(C \cup W^{\prime}\right)\right.$ $\left.\cap R^{\prime}\right)$. Hence $(W-C) \cap R^{\prime} \subseteq Q$, so $(W-C) \cap R^{\prime}$ is within $W^{\prime}=W^{\prime} \cap$ $R^{\prime}$. If $B$ is any atom of $\left(\mathscr{P}_{2}, \mathscr{B}_{2}\right) \cap R^{\prime}$, then we have shown that $B$ is within $(W-C) \cap R^{\prime}$, hence $B$ is within $W^{\prime}$. Let $B$ be an atom of $\left(\mathscr{P}_{1}, \mathscr{B}_{1}\right) \cap R^{\prime}$. If $Q \cap B=\varnothing$, then as $\left(\mathscr{P}_{1}, \mathscr{B}_{1}\right) \cap R^{\prime} \simeq\left(\mathscr{P}_{1}, \mathscr{B}_{1}\right)$, we can choose such a $B$ which is an outermost atom of $\left(\mathscr{P}_{1}, \mathscr{B}_{1}\right) \cap R^{\prime}$. Hence $G=\left(E \cap R^{\prime}\right)-B$ is r.e. By Theorem 2.1, we separate $Q$ and $G$ to obtain a recursive set $T \subseteq Q$ such that $T \cap B=\varnothing$. We now note that $(\mathscr{P}, \mathscr{B}) \cap\left(R^{\prime}-T\right)$ has smaller degree than $(\mathscr{P}, \mathscr{B})$, and that $(\mathscr{P}, \mathscr{B}) \cap(R \cup T)$ either is not irreducible or is not isomorphic to $(\mathscr{P}, \Re)$. (The argument for the latter is the same as the one that showed this fact for $R$ replacing $R \cup T$.) This is impossible as $\left(\mathscr{P}_{1}, \mathscr{B}_{1}\right)$ and $\left(\mathscr{P}_{2}, \mathscr{B}_{2}\right)$ witness the strong irreducibility of $(\mathscr{P}, \mathscr{B})$. Hence $Q \cap B \neq \varnothing$ and so $B$ is within $W^{\prime}$.

Theorem 4.4 (TyPe 2 Refinement Theorem). Let $(\mathcal{P}, \Re)$ be strongly irreducible of type 2 and let $\left(\mathscr{P}_{1}, \mathscr{B}_{1}\right)$ and $\left(\mathscr{P}_{2}, \mathscr{B}_{2}\right)$ witness the strong irreducibility of $(\mathscr{P}, \mathscr{B})$. Let $\left(\mathscr{P}^{*}, \mathscr{B}^{*}\right)$ be the minimizer refinement of $(\mathscr{P}, \mathscr{B})$ for $\left(\mathscr{P}_{2}, \mathscr{B}_{2}\right)$. Let $\left(L^{*}, A^{*}\right) \subseteq\left(L^{\prime \prime}, A^{\prime \prime}\right)$ be finite d-lattices such that $\left(\mathscr{P}^{*}, \mathscr{B}^{*}\right) \simeq$ ( $\left.L^{*}, A^{*}\right)$. Suppose that (3.3), (3.4), (3.6), (3.7), (3.8), and (3.10) hold for ( $\left.L^{*}, A^{*}\right)$ replacing $\left(L^{\prime}, A^{\prime}\right)$. Let $S=\left\{a_{2} \in A^{\prime \prime}: a_{2}\right.$ is an outermost atom of ( $\left.L^{\prime \prime}, A^{\prime \prime}\right)$ contained in an innermost atom of $\left.\left(L^{*}, A^{*}\right)\right\}$ and let $T \subseteq S$. Then there exists a realization $\left(\mathscr{P} ", \mathscr{B}^{\prime \prime}\right)$ of $\left(L^{\prime \prime}, A^{\prime \prime}\right)$ such that the diagram

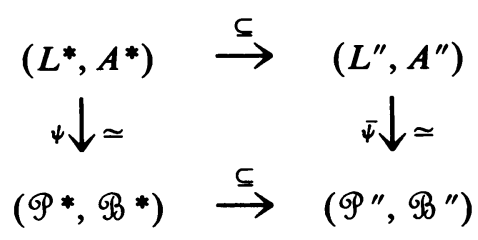

FIGURE 4.2

commutes. Furthermore, for all $b_{2} \in A^{\prime \prime}, \bar{\psi}\left(b_{2}\right)$ is maximal if and only if $b_{2}^{\prime} \in T$.

Proof. The proof follows the following diagram. 


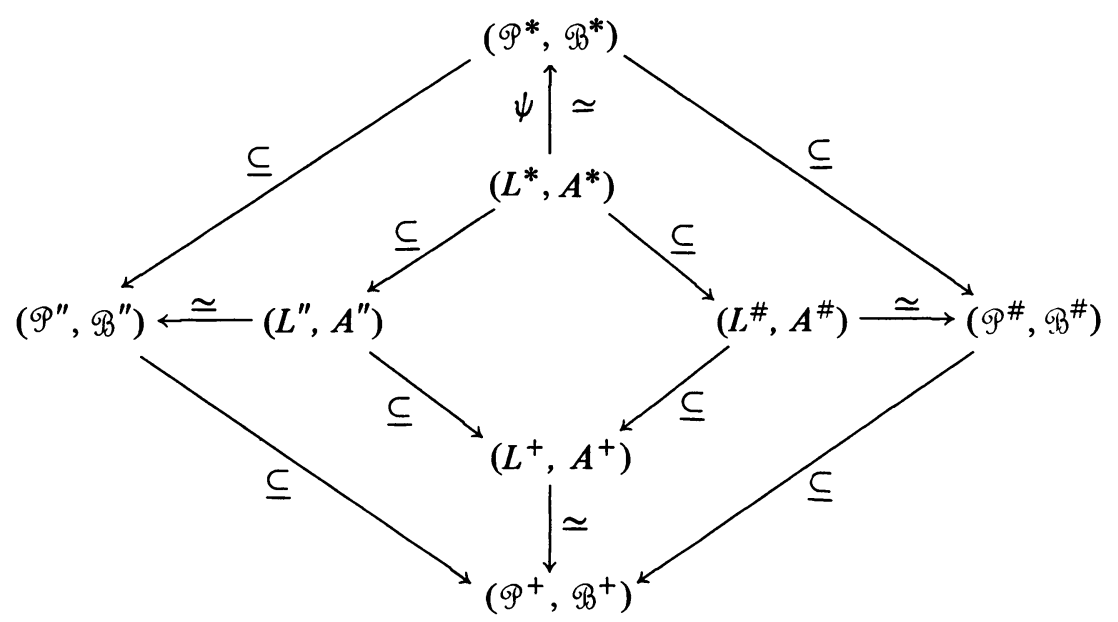

Figure 4.3

Choose $D$ and $E$ such that $\left(\mathscr{P}_{2}, \mathscr{B}_{2}\right)=(\mathscr{P}, \mathscr{B}) \cap D$ and $\left(\mathscr{P}_{1}, \mathscr{B}_{1}\right)=$ $(\mathscr{P}, \mathscr{B}) \cap E$. Let $a^{*}$ be the outermost atom of $\left(L^{*}, A^{*}\right)$. By (3.4), fix the unique outermost atom $a^{2}$ of $\left(L^{\prime \prime}, A^{\prime \prime}\right)$ such that $a^{2} \subseteq a^{*}$ and $L^{\prime \prime}\left(a^{2}\right) \cap a^{* \prime} \neq$ 0. Let $G=\left\{b_{2}:\left(\exists b_{1}\right)\left(b_{2} \subseteq b_{1}\right.\right.$ and $\psi\left(b_{1}\right)$ is an outermost atom of $\left.\left(\mathscr{P}_{1}, \mathscr{B}_{1}\right)\right)$ and $b_{2}$ is an outermost atom of $\left.\left(L^{\prime \prime}, A^{\prime \prime}\right) \cap \psi^{-1}(D)\right\}$. Let $H=\left\{b_{2} \in G: b_{2}\right.$ is just within $\left.a^{2}\right\}=\left\{d_{1}, \ldots, d_{n}\right\}$. We define a refinement $\left(L^{+}, A^{+}\right)$of $\left(L^{\prime \prime}, A^{\prime \prime}\right)$ obtained by partitioning $a^{2}$ into $n+1$ atoms $a_{0}, \ldots, a_{n}$. We let $L^{+}\left(a_{0}\right)=$ $L^{\prime \prime}\left(a^{2}\right)$ and $L^{+}\left(a_{i}\right)=L^{\prime \prime}\left(d_{i}\right) \cup a_{i}$ for $i=1, \ldots, n$. Clearly $\left(L^{\prime \prime}, A^{\prime \prime}\right) \mid a^{* \prime}=$ $\left(L^{+}, A^{+}\right) \mid a^{* \prime}$. Let $\left(L^{\sharp}, A^{\sharp}\right)$ be the $d$-lattice such that $\left(L^{*}, A^{*}\right) \subseteq\left(L^{\sharp}, A^{\sharp}\right) \subseteq$ $\left(L^{+}, A^{+}\right)$and $\left(L^{\sharp}, A^{\sharp}\right)$ is generated by the elements of $L^{*}$ and $a_{0}^{\prime}$. Let $\left(\mathscr{P}^{\#}, \mathscr{B}^{\#}\right)$ be an irreducible preliminary refinement of $(\mathscr{P}, \mathscr{B})$ extending $\left(\mathscr{P}^{*}, \mathscr{B}^{*}\right)$; such a $\left(\mathscr{P}^{\#}, \mathscr{B}^{\#}\right)$ exists by Theorem 4.3 . It is easily verified that $\left(\mathscr{P}^{\#}, \mathscr{B}^{\#}\right) \simeq\left(L^{\#}, A^{\#}\right)$ which is separated.

To prove the theorem, it suffices to show that there is a refinement $\left(\mathscr{P}^{+}, \mathfrak{B}^{+}\right)$of $\left(\mathscr{P}^{\#}, \mathscr{B}^{\#}\right)$ such that the diagram

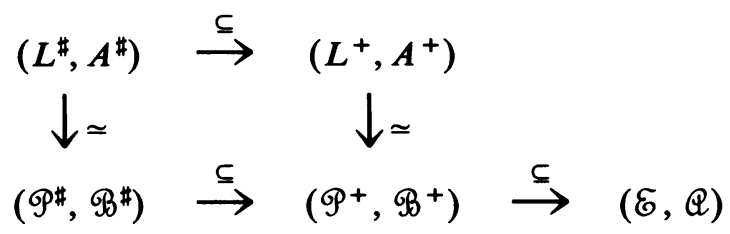

\section{FIGURE 4.4}

commutes, where $\left(\mathscr{P}^{+}, \mathscr{B}^{+}\right)$has no finite atoms, and the maximal sets in $\left(\mathscr{P}^{+}, \mathscr{B}^{+}\right)$are exactly those specified in the theorem. For then we could find $\left(\mathscr{P}^{\prime \prime}, \mathscr{B}^{\prime \prime}\right)$ with no finite atoms such that $\left(\mathscr{P}^{*}, \mathscr{B}^{*}\right) \subseteq\left(\mathscr{P}^{\prime \prime}, \mathscr{B}^{\prime \prime}\right) \subseteq\left(\mathscr{P}^{+}, \mathscr{B}^{+}\right)$ and Figure 4.3 commutes.

The existence of $\left(\mathscr{P}^{+}, \mathscr{B}^{+}\right)$such that Figure 4.4 commutes will follow from Theorem 4.1. We need only verify that (4.1)-(4.4) are satisfied; the maximal- 
ity conditions follow since $\left.\left(\mathscr{P}^{\prime \prime}, \mathscr{B}^{\prime \prime}\right)\right|_{\psi\left(a^{*}\right)}=\left.\left(\mathscr{P}_{1}^{\#}, \mathscr{B}_{1}^{\#}\right)\right|_{\psi\left(a^{*}\right)}$.

(4.1) clearly holds since $a_{0}$ is the unique outermost atom of $\left(L^{\sharp}, A^{\sharp}\right)$ and $a_{0}$ is an atom of $\left(L^{+}, A^{+}\right)$. Let $a, b$ be atoms of $\left(L^{\#}, A^{\sharp}\right)$ such that $a \prec_{j} b$ and $a$ is not innermost. Let $c_{1} \subseteq a$ be an atom of $\left(L^{+}, A^{+}\right)$. If $a \nsubseteq a^{*}$, then by (3.6), there is an atom $b_{1} \subseteq b$ of $\left(L^{+}, A^{+}\right)$such that $c_{1}<b_{1}$. Suppose that $a \subseteq a^{*}$. Then we must have $b=a_{0}$ and $a=a^{*}-a_{0}$. If $c_{1}=a_{i}$ for some $i=$ $1, \ldots, n$, then $a_{i} \in L^{+}\left(a_{0}\right)$. Otherwise, $c_{1}$ is an atom of $\left(L^{*}, A^{*}\right)$ in which case we already have $c_{1} \subseteq L^{+}\left(a_{0}\right)$ from (3.6). Hence (4.2) holds.

Let $a, b$ be atoms of $\left(L^{\sharp}, A^{\sharp}\right)$ such that $a \prec_{j} b$. Let $b_{1} \subseteq b$ be an atom of $\left(L^{+}, A^{+}\right)$. By (3.7), there will be an atom $c_{1} \subseteq a$ of $\left(L^{+}, A^{+}\right)$such that $c_{1} \prec b_{1}$ unless $b_{1}=a_{0}$ or $b_{1}=a_{i}$ for some $i=1, \ldots, n$. (Note that if $\psi(a) \in \mathscr{B}_{1}$, then $a$ is not within $a^{*}-a_{0}$.) If $b_{1}=a_{0}$, then every atom of $\left(L^{+}, A^{+}\right)$is contained in $L^{+}\left(b_{1}\right)$, so there must be an atom $c_{1} \subseteq a$ of $\left(L^{+}, A^{+}\right)$such that $c_{1} \prec b_{1}$. If $b_{1}=a_{i}$ for some $i \neq 0$, then since $\left(L^{\#}, A^{\#}\right)$ is separated and since by $(3.10)\left(L^{\#}, A^{\sharp}\right) \cap\left(L^{+}\left(b_{1}\right) \cap a^{* \prime}\right) \simeq\left(\mathscr{P}_{2}, \mathscr{B}_{2}\right)$, we must have $d_{i} \subseteq a$ and so can choose $c_{1}=d_{i}$ to obtain $c_{1} \prec b_{1}$. Hence (4.3) holds.

Let $c_{1}, b_{1}$ be atoms of $\left(L^{+}, A^{+}\right)$such that $c_{1} \prec_{j} b_{1}$. Let $a, b$ be the atoms of ( $L^{\sharp}, A^{\sharp}$ ) such that $c_{1} \subseteq a, b_{1} \subseteq b$. By (3.8), $a=b$ or $a \prec_{j} b$ unless either $b=a_{0}$ and $a=a_{i}$ for some $i=1, \ldots, n$, or $b=a_{i}$ for some $i=1, \ldots, n$. If $b=a_{0}$ and $a=a_{i}$ for some $i=1, \ldots, n$, then $a_{i} \prec_{j} b$. If $b=a_{i}$ for some $i=1, \ldots, n$, then $\psi(a)$ must be an outermost atom of $\left(\mathscr{P}_{2}, \mathscr{B}_{2}\right)$ in which case $a \prec_{j} b$. Hence (4.4) holds.

Let $\left(L^{\prime}, A^{\prime}\right)$ have $m$ components. Then $(\mathscr{P}, \mathscr{B})$ is said to be of type 3 for $\left(L^{\prime}, A^{\prime}\right)$ if there are pairwise disjoint recursive sets $R_{1}, \ldots, R_{m}$ such that for $i, j \in\{1, \ldots, m\}, R_{i} \cap C^{\prime}$ is not r.e. or cohesive, $(\mathscr{P}, \mathscr{B}) \cap R_{i}$ and $(\mathscr{P}, \mathscr{B})$ $\cap R_{j}$ are equivalent over $(\mathscr{P}, \mathscr{B})$ through the inclusion embedding (i.e., $\psi$ and $\xi$ in Figure 1.1 are given by inclusion).

Let $(\mathscr{P}, \mathscr{B})$ be irreducible of type 3 for $\left(L^{\prime}, A^{\prime}\right)$ with companion $\left(\mathscr{P}_{2}, \mathscr{B}_{2}\right)$ $=(\mathscr{P}, \mathscr{B}) \cap E$ and such that $D=C-E$ is r.e. Since $\left(\mathscr{P}_{2}, \mathscr{B}_{2}\right)$ is a companion of $(\mathscr{P}, \mathscr{B}),(\mathscr{P}, \mathscr{B}) \cap C \simeq(\mathscr{P}, \mathscr{B}) \cap D$. Let $\left(\mathscr{P}^{*}, \mathscr{B}^{*}\right)$ be the refinement of $(\mathscr{P}, \mathscr{B})$ generated by $\{X: X \in \mathscr{P}$ or $X=D$ or $X=E\}$. Note that $\left(\mathscr{P}^{*}, \mathscr{B}^{*}\right)$ is a companion refinement of $(\mathscr{P}, \mathscr{B})$. Let $R_{1}, \ldots, R_{m}$ be as in the preceding paragraph. Let $R$ be the complement of $\cup\left\{R_{i}: 2<i<m\right\}$. Let $\left(\mathscr{P}^{\#}, \mathscr{B}^{\sharp}\right)$ be the refinement of $(\mathscr{P}, \mathscr{B})$ generated by $\left\{X: X \in \mathscr{P}\right.$ or $X=R_{i}$ for some $i=1, \ldots, m\} .\left(\mathscr{P}^{\#}, \mathscr{B}^{\sharp}\right)$ is said to be a preliminary refinement of $(\mathscr{P}, \mathscr{B})$ of type 3 if $\left(\mathscr{P}^{\#}, \mathscr{B}^{\#}\right) \cap R \simeq(\mathscr{P}, \mathscr{B})$. Note that if $\left(\mathscr{P}^{\sharp}, \mathscr{B}^{\#}\right)$ is a preliminary refinement of $(\mathscr{P}, \mathscr{B})$ of type 3 , then if we choose $D=R \cap C$ and $E=R^{\prime} \cap C$, and form $\left(\mathscr{P}^{*}, \mathscr{B}^{*}\right)$ as above, then $\left(\mathscr{P}^{*}, \mathscr{B}^{*}\right)$ is a companion refinement of $(\mathscr{P}, \mathscr{B})$ induced by $\left(\mathscr{P}^{\#}, \mathfrak{B}^{\#}\right)$.

Theorem 4.5 (Type 3 Preliminary Refinement Theorem). Let $k$ be the number of nonequivalent sub-d-lattices of $(\mathcal{E}, \mathcal{Q})$ embeddable into $(\mathscr{P}, \mathscr{B})$ under inclusion, and let $m$ be the number of components of $\left(L^{\prime}, A^{\prime}\right)$. Suppose that $C$ is not quasi-r-maximal of degree $<2 m(k-1)$, but that $C$ is simple. Then $(\mathscr{P}, \mathscr{B})$ is of type 3 for $\left(L^{\prime}, A^{\prime}\right)$. Furthermore, if $(\mathscr{P}, \mathscr{B})$ is irreducible, then $(\mathscr{P}, \mathscr{B})$ has an irreducible preliminary refinement of type 3. 
Proof. Since $C$ is not quasi-r-maximal of rank $<2 m(k-1)$, there are recursive sets $R_{0}, \ldots, R_{2 m(k-1)}$ such that for all $i<2 m(k-1), R_{i} \cap C^{\prime}$ is infinite. Without loss of generality, we may assume that $(\mathscr{P}, \mathscr{B}) \cap R_{i}$ is irreducible for all $i<2 m(k-1)$, else the reduction will yield the desired sets. By the pigeonhole principle, there are pairwise disjoint recursive sets $S_{1}, \ldots, S_{2 m} \subseteq\left\{R_{0}, \ldots, R_{2 m(k-1)}\right\}$ such that for all $j, n<2 m,(\mathscr{P}, \mathscr{B}) \cap S_{j}$ is equivalent to $(\mathscr{P}, \mathscr{B}) \cap S_{n}$ over $(\mathscr{P}, \mathscr{B})$ under the inclusion embedding. Fix $j \in\{1, \ldots, m\}$. Let $T_{j}$ be the fusion of $(\mathscr{P}, \mathscr{B}) \cap S_{2 j-1}$ and $(\mathscr{P}, \mathscr{B}) \cap$ $S_{2 j}$ over $(\mathscr{P}, \mathscr{B}) \cap S_{2 j}$ under the isomorphism embedding. Then $(\mathscr{P}, \mathscr{B}) \cap T_{j}$ is equivalent to $(\mathscr{P}, \mathscr{B}) \cap S_{1}$ over $(\mathscr{P}, \mathscr{B}) \cap S_{1}$ under the isomorphism embedding, and $C^{\prime} \cap T_{j}$ is not cohesive. As $C$ is simple, $T_{j} \cap C^{\prime}$ is not r.e. Hence $(\mathscr{P}, \mathscr{B})$ is of type 3 for $\left(L^{\prime}, A^{\prime}\right)$ with companion isomorphic to $(\mathscr{P}, \mathscr{B}) \cap\left(T_{j} \cap C\right)$. It is easily checked that $(\mathscr{P}, \mathscr{B}) \cap T_{j}$ is irreducible.

Let $T$ be the complement of $\cup\left\{T_{j}: 2<j<m\right\}$. We must show that $(\mathscr{P}, \mathscr{B}) \cap T \simeq(\mathscr{P}, \mathscr{B})$ and that $(\mathscr{P}, \mathscr{B}) \cap T$ is irreducible. $(\mathscr{P}, \mathscr{B}) \cap T$ is the fusion of $(\mathscr{P}, \mathscr{B}) \cap T_{1}$ and $(\mathscr{P}, \mathscr{B}) \cap\left(T \cap T_{1}^{\prime}\right)$ over $(\mathscr{P}, \mathscr{B}) \cap T$ under the inclusion embedding. Since $(\mathscr{P}, \mathscr{B}) \cap T_{1}$ is equivalent to $(\mathscr{P}, \mathscr{B}) \cap\left(T^{\prime}\right.$ $\left.\cup T_{1}\right)$ over $(\mathscr{P}, \mathscr{B})$ under the inclusion embedding, the fusion of $(\mathscr{P}, \mathscr{B}) \cap$ $\left(T \cap T_{1}^{\prime}\right)$ and $(\mathscr{P}, \mathscr{B}) \cap T_{1}$ over $(\mathscr{P}, \mathscr{B}) \cap T_{1}$ is isomorphic to the fusion of $(\mathscr{P}, \mathscr{B}) \cap\left(T \cap T_{1}^{\prime}\right)$ and $(\mathscr{P}, \mathscr{B}) \cap\left(T^{\prime} \cup T_{1}\right)$ over $(\mathscr{P}, \mathscr{B})$, where both fusions are under the isomorphism embedding. But the latter fusion is $(\mathcal{P}, \mathscr{B})$. So $(\mathscr{P}, \mathscr{B}) \cap T \simeq(\mathscr{P}, \mathscr{B})$. Let $R \subseteq T$ be any recursive set containing $T \cap$ $C^{\prime}$. Let $R^{*}=R \cup \cup\left\{T_{i}: 2<i<m\right\}$. Then since $(\mathscr{P}, \mathscr{B}) \cap T_{1}$ is irreducible and hence $(\mathscr{P}, \mathscr{B}) \cap\left(R \cap T_{1}\right)$ is equivalent to $(\mathscr{P}, \mathscr{B}) \cap\left(R^{*}-(R-\right.$ $\left.T_{1}\right)$ ) over $(\mathscr{P}, \mathscr{B})$ under the inclusion embedding, we see by the same argument as above that $(\mathscr{P}, \mathscr{B}) \cap R \simeq(\mathscr{P}, \mathscr{B}) \cap R^{*}$. Since $(\mathscr{P}, \mathscr{B})$ is irreducible and $C^{\prime} \subseteq R^{*}$, $(\mathscr{P}, \mathscr{B}) \cap R^{*} \simeq(\mathscr{P}, \mathscr{B})$. Hence $(\mathscr{P}, \mathscr{B}) \cap R \simeq$ $(\mathscr{P}, \mathscr{B})$ and so $(\mathscr{P}, \mathscr{B}) \cap T$ is irreducible.

TheOREM 4.6 (TYPe 3 REFINEMENT ThEOREM). Let ( $\mathcal{P}, \mathscr{B})$ be irreducible and of type 3 for $\left(L^{\prime}, A^{\prime}\right)$. Let $\left(L^{\prime}, A^{\prime}\right)$ have exactly $k$ components which intersect the outermost atom $a_{0}$ of $(L, A)$, say $\left(L^{\prime}, A^{\prime}\right) \mid d_{i}$ for $i=1, \ldots, k$ such that $\left(L^{\prime}, A^{\prime}\right)\left|\left(d_{i} \cap a_{0}^{\prime}\right) \simeq(L, A)\right| a_{0}^{\prime}$ and $\left(L^{\prime}, A^{\prime}\right) \cap\left(d_{j} \cap a_{0}^{\prime}\right)$ is equivalent to $\left(L^{\prime}, A^{\prime}\right) \mid\left(d_{i} \cap a_{0}^{\prime}\right)$ over $(L, A)$ under the inclusion embedding for $i, j \in$ $\{2, \ldots, k\}$. Let $\left(\mathcal{P}^{\#}, \mathscr{B}^{\sharp}\right)$ be an irreducible preliminary refinement of $(\mathscr{P}, \mathscr{B})$ with companion $\left(\mathscr{P}_{2}, \mathscr{B}_{2}\right) \simeq\left(L^{\prime}, A^{\prime}\right) \cap\left(d_{2} \cap a_{0}^{\prime}\right)$ (replace $d_{2}$ with $d_{1}$ if $\left.k=1\right)$, and let $\left(\mathscr{P}^{*}, \mathscr{B}^{*}\right)$ be the companion refinement of $\left(\mathscr{P}, \mathscr{B}^{\prime}\right)$ induced by $\left(\mathscr{P}^{\#}, \mathscr{B}^{\#}\right)$. Let $\left(L^{*}, A^{*}\right)$ and $\left(L^{\sharp}, A^{\sharp}\right)$ be given so that the diagram

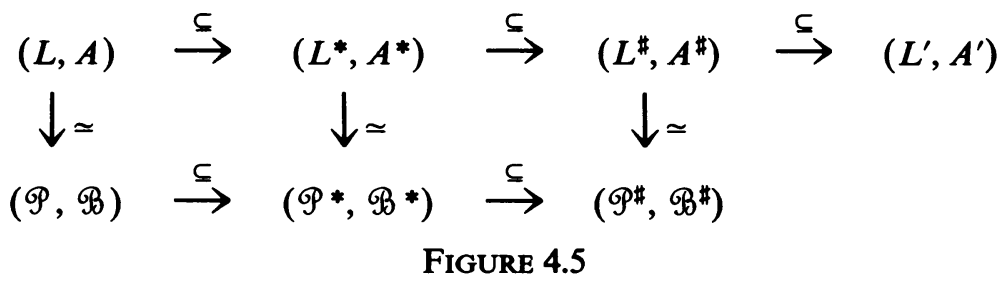

commutes. Assume that (3.3), (3.5), (3.6), (3.7), and (3.8) are satisfied by 
$\left(L^{*}, A^{*}\right) \subseteq\left(L^{\prime}, A^{\prime}\right)$. Let $S=\left\{a_{1} \in A^{\prime}: a_{1}\right.$ is an outermost atom of $\left(L^{\prime}, A^{\prime}\right)$ contained in an innermost atom of $\left.\left(L^{*}, A^{*}\right)\right\}$, and let $T \subseteq S$. Then there is a realization $\left(\mathcal{P}^{\prime}, \mathfrak{B}^{\prime}\right)$ of $\left(L^{\prime}, A^{\prime}\right)$ such that the diagram

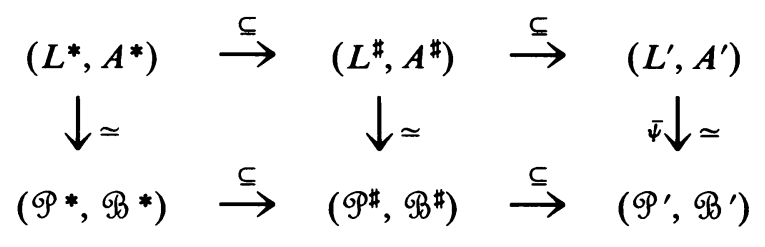

FIGURE 4.6

commutes, and for all $b_{1} \in A^{\prime}, \bar{\psi}\left(b_{1}\right)$ is maximal if and only if $b_{1}^{\prime} \in T$.

Proof. It suffices to verify the conditions (4.1)-(4.4) for $\left(L^{\sharp}, A^{\sharp}\right) \subseteq$ $\left(L^{\prime}, A^{\prime}\right)$, as then the result will follow from Theorem 4.1 as $\left(\mathscr{P}^{\sharp}, \mathscr{B}^{\sharp}\right)$ is separated, noting that since $\left(\mathscr{P}^{\#}, \mathscr{S}^{\#}\right)$ is a preliminary refinement of $(\mathscr{P}, \mathscr{B})$, no outermost atom of $\left(\mathscr{P}^{\sharp}, \mathscr{B}^{\sharp}\right)$ can be cohesive, hence by (4.1), no outermost atom of $\left(\mathscr{P}^{\prime}, \mathscr{B}^{\prime}\right)$ which is contained in $a_{0}$ can be cohesive.

(4.1) is clearly satisfied from the hypotheses of the theorem as $\left(\mathscr{P}^{\#}, \mathscr{B}^{\#}\right)$ is a preliminary refinement of $(\mathcal{P}, \Re)$. Let $a, b$ be atoms of $\left(L^{\sharp}, A^{\sharp}\right)$ such that $a \prec_{j} b$ and $a$ is not innermost. Let $a_{1} \subseteq a$ be an atom of $\left(L^{\prime}, A^{\prime}\right)$. Let $c, d$ be the atoms of $\left(L^{*}, A^{*}\right)$ such that $a \subseteq c, b \subseteq d$. As $\left(L^{*}, A^{*}\right) \subseteq\left(L^{\sharp}, A^{\sharp}\right), c$ is within $d$. By iterating applications of (3.6), there is an atom $b_{1} \subseteq d$ of $\left(L^{\prime}, A^{\prime}\right)$ such that $a_{1} \prec b_{1}$. But then $a_{1}$ and $b_{1}$ must lie in the same component of ( $\left.L^{\prime}, A^{\prime}\right)$, and $b$ is the intersection of $d$ with this component, so $b_{1} \subseteq b$. Hence (4.2) holds.

Let $a, b$ be atoms of $\left(L^{\#}, A^{\#}\right)$ such that $a \prec_{j} b$. Let $b_{1} \subseteq b$ be an atom of $\left(L^{\prime}, A^{\prime}\right)$. Let $c, d$ be the atoms of $\left(L^{*}, A^{*}\right)$ such that $a \subseteq c, b \subseteq d$. Let $a^{2}$ be the atom of $\left(L^{\prime}, A^{\prime}\right)$ as in (3.5). Note that if $b \subseteq a_{0}$ and $b_{1} \neq a^{2}$, then $b_{1}$ is an outermost atom of $\left(L^{\sharp}, A^{\sharp}\right)$ and if $\left(L_{1}, A_{1}\right)=\left((L, A) \cap a_{0}^{\prime}\right)-\left(L_{2}, A_{2}\right)$ $\left(\left(L_{1}, A_{1}\right)\right.$ is just the complementary part of $(L, A) \cap a_{0}^{\prime}$ to the part isomorphic to the companion), then $a \cap e=0$ for all $e \in A_{1}$. Hence by iterating applications of (3.7), there is an atom $a_{1} \subseteq c$ such that $a_{1} \prec b_{1} . a_{1}$ and $b_{1}$ must, therefore, lie in the same component of $\left(L^{\prime}, A^{\prime}\right)$, and $a$ is the intersection of $c$ with this component. Thus $a_{1} \subseteq a$, and (4.3) holds.

Let $a_{1}, b_{1}$ be atoms of $\left(L^{\prime}, A^{\prime}\right)$ such that $a_{1} \prec_{j} b_{1}$. Let $a, b$ be the atoms of ( $\left.L^{\sharp}, A^{\sharp}\right)$ such that $a_{1} \subseteq a, b_{1} \subseteq b$. Let $c, d$ be the atoms of $\left(L^{*}, A^{*}\right)$ such that $a \subseteq c, b \subseteq d$. As in the previous paragraph, we see that (3.8) has no restrictions in this case, so by (3.8), $c=d$ or $c \prec_{j} d$. Since $a_{1}$ is within $b_{1}$, $a=b$ or $a$ is within $b$. As $a \subseteq c, b \subseteq d$, we must have $a=b$ if $c=d$ since then both $a$ and $b$ would be the intersection of $c$ with the same component. And if $c \prec_{j} d$ and $\left(L^{\#}, A^{\#}\right)$ is a refinement of $\left(L^{*}, A^{*}\right)$, then as $a$ is within $b$, $a \prec_{j} b$. Hence (4.4) holds.

We are now ready to give the decision procedure. Let $\sigma \equiv(\forall \bar{x})(\exists \bar{y}) R(\bar{x}, \bar{y})$ be a sentence of our language. Recall that quantifiers range only over r.e. sets. Then $\sigma$ is uniformly effectively equivalent to a conjunction of sentences of the 
form $\sigma_{1} \equiv(\forall \bar{x})(\exists \bar{y})\left(D(\bar{x}) \rightarrow \bigvee_{i} P_{i}(\bar{x}, \bar{y})\right)$ where $D$ and all the $P_{i}$ are finite diagrams and $D \subseteq P_{i}$ for all $i$. Hence it suffices to decide all sentences of the form $\sigma_{1}$. By Lemma $1.3, \sigma_{1}$ is uniformly effectively equivalent to a conjunction of sentences of the form $\sigma_{2} \equiv(\forall \bar{x})(\exists \bar{y})\left(E(\bar{x}) \rightarrow \bigvee_{i} Q_{i}(\bar{x}, \bar{y})\right)$ where $E$ and $Q_{i}$ are the finite separated diagrams of the $d$-lattices $(L, A),\left(L_{i}, A_{i}\right)$ respectively, and $E$ has characteristic less than or equal to that of $D$ and $(L, A) \subseteq\left(L_{i}, A_{i}\right)$ for all $i$. Hence it suffices to decide all sentences of the form $\sigma_{2}$. Furthermore, $\sigma_{2}$ is true if and only if for every realization $(\mathscr{P}, \mathscr{B})$ of $(L, A)$, there is an $i$ and a realization $\left(\mathscr{P}_{i}, \mathscr{B}_{i}\right)$ of $\left(L_{i}, A_{i}\right)$ such that the diagram

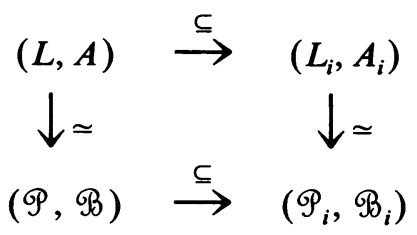

FIGURE 4.7

commutes, with appropriate sets maximal.

We note that if two components of $(\mathscr{P}, \mathscr{B})$ are equivalent over $(\mathscr{P}, \mathscr{B})$ through the inclusion embedding, then their fusion over the first component under isomorphism is equivalent under inclusion over $(\mathscr{P}, \mathscr{B})$ to each of the components. Furthermore, if one of the components is irreducible, so is their fusion. Since the ordering of characteristics is a well-ordering, and since we can effectively list the finite number of nonequivalent embeddings of $d$-lattices into $(\mathscr{P}, \mathscr{B})$, we can effectively list the finite number of possible combinations of components for $(\mathscr{P}, \mathscr{B})$ such that in each combination, each component is irreducible and embeddable into $(\mathcal{P}, \mathscr{B})$, no two components are equivalent over $(\mathscr{P}, \mathscr{B})$ under inclusion, and the fusion of the components over $(\mathscr{P}, \mathscr{B})$ under the inclusion map is $(\mathscr{P}, \mathscr{B})$. By Lemma 1.2 and induction, it thus suffices to decide whether there is an $i$ such that Figure 4.7 commutes with appropriate elements of $\mathscr{P}_{i}$ maximal, given that $(\mathscr{P}, \mathscr{B})$ is irreducible with one component. Hence we assume that $(\mathscr{P}, \mathscr{B})$ is irreducible with one component.

Let $C^{\prime}$ be the outermost atom of $(\mathscr{P}, \mathscr{B})$. Then $C$ is of type 1 , type 2 , type 3 , or quasimaximal. (The type of $C$ is just the type of $(\mathscr{P}, \mathscr{B})$.)

First consider the case where $C$ is of type 1 . By the results of $\S 3$, there must exist an $i$ such that (3.3), (3.6), (3.7), (3.8), and (3.9) hold for $(L, A) \subseteq\left(L_{i}, A_{i}\right)$. Theorem 4.2 shows these conditions to be sufficient, so we have a decision procedure for $C$ of type 1 .

Consider the case where $C$ is of type 2 . Let $\left(\mathscr{P}_{2}, \mathscr{B}_{2}\right)$ be a minimizer for $(\mathscr{P}, \mathscr{B})$ with associated cominimizer $\left(\mathscr{P}_{1}, \mathscr{B}_{1}\right)$. We can effectively list the finite number of possible combinations of components for $(\mathscr{P}, \mathscr{B})$ such that each component has smaller degree than $(\mathscr{P}, \mathscr{B})$ and no two components of the same degree (if degree is defined for the components) are equivalent over 
$(\mathscr{P}, \mathscr{B})$ under inclusion. Since the ordering of degrees is well founded, it suffices by Lemma 1.2 to consider the case where $(\mathscr{P}, \mathscr{B})$ is strongly irreducible as witnessed by $\left(\mathscr{P}_{1}, \mathscr{B}_{1}\right)$ and $\left(\mathscr{P}_{2}, \mathscr{B}_{2}\right)$. The minimizer refinement $\left(\mathscr{P}^{*}, \mathscr{B}^{*}\right)$ of $(\mathscr{P}, \mathscr{B})$ for $\left(\mathscr{P}_{1}, \mathscr{P}_{1}\right)$ and $\left(\mathscr{P}_{2}, \mathscr{P}_{2}\right)$ is then uniquely determined, and we consider $(\mathscr{P}, \mathscr{B})$ with all possible minimizers and associated cominimizers. By the results of $\S 3$, there must exist an $i$ and finite $d$-lattices $\left(L^{*}, A^{*}\right)$ and $\left(L^{\prime \prime}, A^{\prime \prime}\right)$ such that the diagram

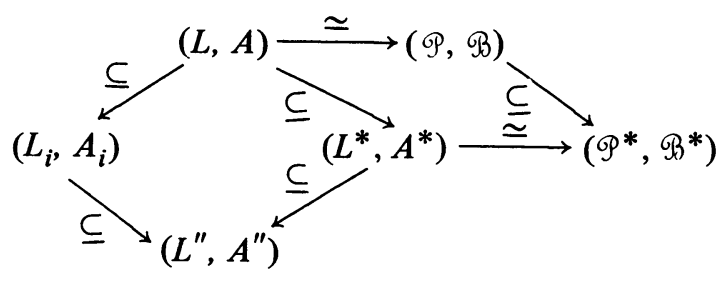

FIgURE 4.8

commutes, and (3.3), (3.4), (3.6), (3.7), (3.8), and (3.10) are satisfied by $\left(L^{*}, A^{*}\right) \subseteq\left(L^{\prime \prime}, A^{\prime \prime}\right)$. The existence of such an $i$ can effectively be determined. By Theorem 4.4, if such an $i$ exists, then Figure 4.7 commutes for that $i$ with the appropriate sets maximal, so we have a decision procedure for $C$ of type 2 .

Consider the case where $(\mathscr{P}, \mathscr{B})$ is of type 3 but not of type 2 with companion $\left(\mathscr{P}_{2}, \mathscr{B}_{2}\right)$, and let $\left(\mathscr{P}^{*}, \mathscr{B}^{*}\right)$ be the companion refinement of $(\mathscr{P}, \mathscr{B})$ generated by $\left(\mathscr{P}_{2}, \mathscr{B}_{2}\right)$. By the results of $\S 3$, there must exist an $i$ and finite $d$-lattices $\left(L^{*}, A^{*}\right)$ and $\left(L^{\prime \prime}, A^{\prime \prime}\right)$ such that (3.3), (3.5), (3.6), (3.7), and (3.8) are satisfied by $\left(L^{*}, A^{*}\right) \subseteq\left(L^{\prime \prime}, A^{\prime \prime}\right)$ and Figure 4.8 commutes. Furthermore, we can effectively decide whether or not such an $i$ exists. Suppose that such an $i$ exists. Let $\left(L^{\prime \prime}, A^{\prime \prime}\right)$ have exactly $k$ components which have nonempty intersection with the outermost atom of $(L, A)$, say $\left(L^{\prime \prime}, A^{\prime \prime}\right) \mid d_{i}$, $i=1, \ldots, k$. By (3.3) and (3.7), for exactly one of these components, say $\left(L^{\prime \prime}, A^{\prime \prime}\right) \mid d_{1}$ do we have $(L, A) \cap d_{1} \simeq(L, A)$. By (3.5), (3.7), and (3.8), $(L, A) \cap d_{i} \simeq\left(\mathscr{P}_{2}, \mathscr{B}_{2}\right)$ for $i=2, \ldots, k$. Hence there exists a preliminary refinement $\left(\mathscr{P}^{\#}, \mathscr{S}^{\#}\right)$ of $(\mathscr{P}, \mathscr{B})$ inducing a companion refinement isomorphic to $\left(\mathscr{P}^{*}, \mathscr{B}^{*}\right)$ (without loss of generality, we assume equality instead of isomorphism) and a finite $d$-lattice $\left(L^{\#}, A^{\sharp}\right)$ such that the diagram

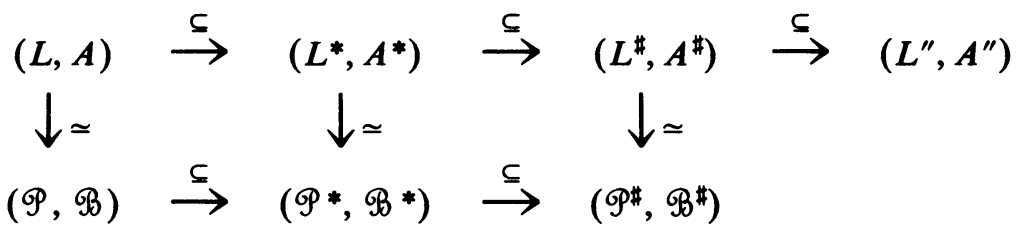

FIGURE 4.9

commutes. Hence by Theorem 4.6, there exists an $i$ such that Figure 4.7 commutes for that $i$ with the appropriate sets maximal, so we have a decision procedure for $C$ of type 3 . 
Let $k$ be the number of components of $(L, A), m$ the number of nonequivalent embeddings of $d$-lattices into $(L, A)$. Our decision procedure for $C$ of type 3 works where $C$ is simple, hence by Theorem 4.5 , for $C$ not quasi-rmaximal of rank $<2 m(k-1)$. If $C$ is quasi-r-maximal of rank $<2 m(k-$ $1)$, then either $C$ is of type 2 , or $C$ is quasimaximal of rank $<2 m(k-1)$. As we have a decision procedure for $C$ of type 2 , it suffices to consider the case where $C$ is quasimaximal of rank $<2 m(k-1)$. But then $(\mathscr{P}, \mathscr{B})$ is the fusion of $<2 m(k-1)$ type 1 components, each embeddable into $(\mathscr{P}, \mathscr{B})$, hence by Lemma 1.2 and the decision procedure for $C$ of type 1 , we have a decision procedure in this last case also.

We note that slight modifications of the above decision procedure will yield a decision procedure for the $\forall \exists$ theorem of $\left(\mathcal{E}^{*}, \mathbb{Q}^{*}\right)$ in our language with the additional one-place predicate Hhs distinguishing the hhs sets. The needed modifications deal only with the way innermost atoms are treated; i.e., the ability to arbitrarily specify whether $\operatorname{Max}(x), \operatorname{Hhs}(x), \sim \operatorname{Max}(x)$, $\sim \operatorname{Hhs}(x)$ for appropriate $x$.

\section{REFERENCES}

1. R. M. Friedberg, Three theorems on recursive enumeration, J. Symbolic Logic 23 (1958), 309-316. MR 22 \# 13; 22 \#2545.

2. A. H. Lachlan, Degrees of recursively enumerable sets which have no maximal superset, J. Symbolic Logic 33 (1968), 431-443. MR 38 \# 4314.

3. __ The elementary theory of recursively enumerable sets, Duke Math. J. 35 (1968), 123-146. MR 37 \#2593.

4. $\quad$ On the lattice of recursively enumerable sets, Trans. Amer. Math. Soc. 130 (1968), 1-37. MR 37 \#2594.

5. __ On some games which are relevant to the theory of recursively emumerable sets, Ann. of Math. (2) 91 (1970), 291-310. MR 44 \# 1562.

6. M. Lerman, On elementary theories of some lattices of $\alpha$-recursively enumerable sets, Ann. Math. Logic 14 (1978), 227-272.

7. M. Lerman, R. A. Shore and R. I. Soare, r-maximal major subsets, Israel J. Math. 31 (1978), 1-18.

8. M. Lerman and R. I. Soare, $d$-simple sets, small sets, and degree classes, Pacific J. Math. (to appear).

9. D. A. Martin, Classes of recursively enumerable sets and degrees of unsolvability, Zeitschrift für Math. Logik und Grundlagen der Math. 12 (1966), 295-310. MR 37 \#68.

10. J. C. Owings, Jr., Recursion, metarecursion, and inclusion, J. Symbolic Logic 32 (1967), 173-179. MR 36 \# 46.

11. R. W. Robinson, Two theorems on hyperhypersimple sets, Trans. Amer. Math. Soc. 128 (1967), 531-538. MR 35 \#6549.

12. J. R. Shoenfield, Degrees of classes of RE sets, J. Symbolic Logic 41 (1976), 695-696.

13. R. A. Shore, Determining automorphisms of the recursively enumerable sets, Proc. Amer. Math. Soc. 65 (1977), 318-326. MR 56 \# 5248

14. Nowhere simple sets and the lattice of recursively enumerable sets, J. Symbolic Logic 43 (1978), 322-330.

15. R. I. Soare, Automorphisms of the lattice of recursively enumerable sets. Part I: Maximal sets, Ann. of Math. (2) 100 (1974), 80-120. MR 50 \# 12685.

Department of Mathematics, Universtty of Connecticut, Storrs, Connecticut 06268

Department of Mathematics, University of Chicago, Chicago, Illinois 60637 